\title{
POLARIZATION TRANSFER IN THE REACTION ${ }^{4} \mathrm{He}\left(\vec{e}, e^{\prime} \vec{p}\right)^{3} \mathrm{H}$ IN THE QUASIELASTIC SCATTERING REGION
}

\author{
BY SONJA DIETERICH
}

\author{
A dissertation submitted to the \\ Graduate School-New Brunswick \\ Rutgers, The State University of New Jersey \\ in partial fulfillment of the requirements \\ for the degree of \\ Doctor of Philosophy \\ Graduate Program in Physics and Astronomy \\ Written under the direction of \\ Professor Ronald D. Ransome \\ and approved by
}

New Brunswick, New Jersey

May, 2002 


\section{ABSTRACT OF THE DISSERTATION}

\section{Polarization Transfer \\ in the Reaction ${ }^{4} \mathrm{He}\left(\vec{e}, e^{\prime} \vec{p}\right)^{3} \mathrm{H}$ in the Quasielastic Scattering Region}

\section{by Sonja Dieterich \\ Dissertation Director: Professor Ronald D. Ransome}

There has been a longstanding issue concerning possible nucleon modifications in a (dense) nuclear medium. Polarization transfer data for exclusive quasielastic electron scattering are a sensitive to the ratio of the electric and magnetic nucleon form factors in the medium. Although proper interpretation of the results requires accounting for such effects as final state interactions and meson exchange currents, their effect on polarization transfer is predicted to be small. Studies of model dependencies, e.g., the off-shell current operator and spinor distortions, have been done. Final results of a measurement of polarization transfer in the ${ }^{4} \mathrm{He}\left(\vec{e}, e^{\prime} \vec{p}\right)^{3} \mathrm{H}$ reaction will be discussed. The experiments were carried out at MAMI, Mainz at a $Q^{2}$ of $0.4 \mathrm{GeV}^{2}$ and at the Thomas Jefferson Lab, Newport News, Virginia at the $Q^{2}$ values $0.5,1.0,1.6$ and 2.6 $\mathrm{GeV}^{2}$. Measured values of the transferred and induced polarizations are compared with various theoretical calculations. The experiment showed a difference between the fully relativistic model which may indicate medium modifications of the form factor. 


\section{Table of Contents}

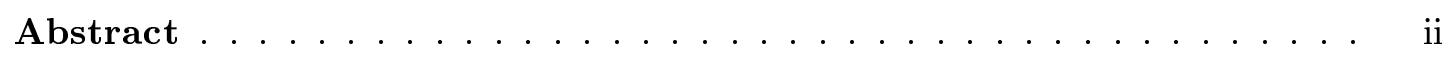

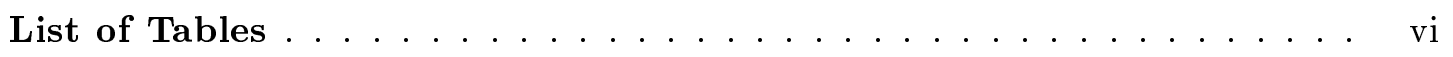

List of Figures $\ldots \ldots \ldots \ldots \ldots \ldots \ldots$ vii

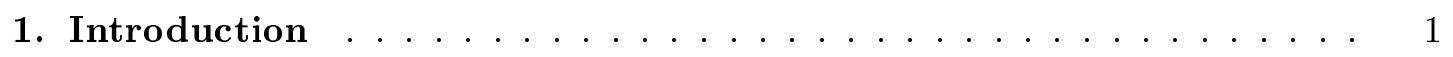

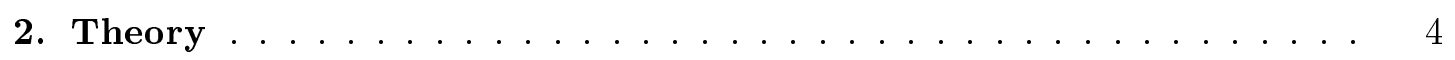

2.1. Electron Scattering . . . . . . . . . . . . . . . 4

2.1.1. Electron-Nucleon Scattering . . . . . . . . . . . . 4

2.1.2. Electron-Nucleus Scattering . . . . . . . . . . . 8

2.2. Theoretical Models and Predictions . . . . . . . . . . . . . . . 11

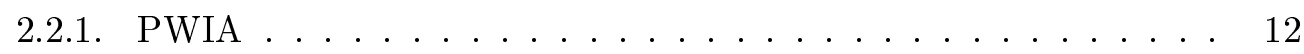

2.2.2. DWIA and RDWIA ................. 13

2.2.3. Two-Body Currents . . . . . . . . . . . . . 18

2.3. Medium Dependence of Nucleon Structure . . . . . . . . . . . . . . 18

2.3.1. Introduction . . . . . . . . . . . . 18

2.3.2. Theoretical Models . . . . . . . . . . . . . . . . . . 19

3. Experimental Setups at MAMI and TJNAF . . . . . . . . . . 24

3.1. Kinematics .......................... 24

3.2. Mainz Experiment A1/2-93 . . . . . . . . . . . . . 25

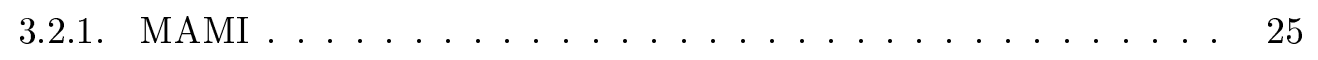

3.2.2. Experimental Setup . . . . . . . . . . . . . 28

3.2.3. Trigger and Data Acquisition . . . . . . . . . . . . . 36 
3.3. Jefferson Lab E93-049 . . . . . . . . . . . . . . . . . 37

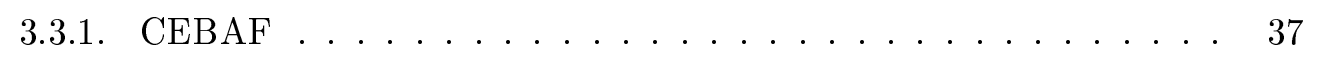

3.3.2. Beam and Beam Diagnostics . . . . . . . . . . . 38

3.3.3. Experimental Setup . . . . . . . . . . . . . . . 40

3.3.4. Trigger and Data Acquisition . . . . . . . . . . . . . 44

4. Raw Data Analysis . . . . . . . . . . . . . . . . . . 47

4.1. Introduction . . . . . . . . . . . . . . . 47

4.2. Event Reconstruction . . . . . . . . . . . . . . . 47

4.2.1. Data Analysis Codes . . . . . . . . . . . . . . . 47

4.2.2. Coordinate Systems . . . . . . . . . . . . . . . 48

4.2.3. Tracking . . . . . . . . . . . . . . . . 49

4.2.4. Energy Loss Correction . . . . . . . . . . . . . . . 50

4.2.5. Vertex Reconstruction ................ 51

4.3. Calibrations ........................... 52

4.3.1. Survey and Spectrometer Alignment . . . . . . . . . . . 52

4.3.2. Alignment of Detectors . . . . . . . . . . . . . 53

4.3.3. Beam Energy and Polarization . . . . . . . . . . . . 59

4.4. Event Selection . . . . . . . . . . . . . . . . . . 62

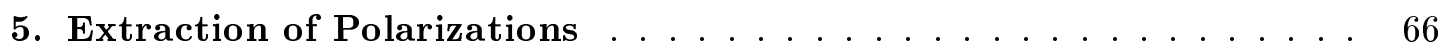

5.1. Introduction to Polarimetry . . . . . . . . . . . . . . 66

5.2. Spin Transport Matrices . . . . . . . . . . . . . . . . 68

5.3. Maximum Likelihood Method . . . . . . . . . . . . . . . . . . 69

5.4. FPP Event Selection . . . . . . . . . . . . . . . 71

5.5. Systematic Uncertainties . . . . . . . . . . . . . . . . 72

6. Results and Discussion . . . . . . . . . . . . . . . 77

6.1. Introduction . . . . . . . . . . . . 77

6.2. Hydrogen Results . . . . . . . . . . . . . . . . . . 77 
6.3. Acceptance averaging . . . . . . . . . . . . . . 78

6.4. Helium Results . . . . . . . . . . . . . . . . . . . . . 80

6.5. Quark-Hadron Duality and the Nuclear EMC Effect . . . . . . . . . 84

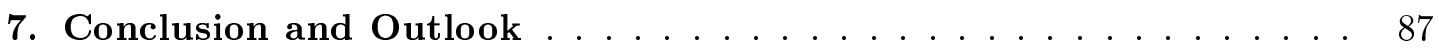

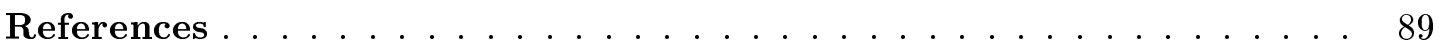




\section{List of Tables}

2.1. Properties of response functions. . . . . . . . . . . . . . . . . . 12

3.1. Table of kinematics. . . . . . . . . . . . . . . . . . 25

3.2. Table of optical properties for spectrometer A and B. . . . . . . . . . 29

3.3. Target parameters during the experiment. . . . . . . . . . . . . . . 41

3.4. Table of optical properties for the HRS. . . . . . . . . . . . . . . . . . 42

4.1. Survey and LVDT results for the HRS. . . . . . . . . . . . . . . . . 52

4.2. Comparison of surveys using hydrogen data. . . . . . . . . . . . . 53

4.3. Spectrometer angles and offsets for the different settings of JLab E93-049. 54

4.4. Adjusted offset parameters for the VDC detector package of the Mainz

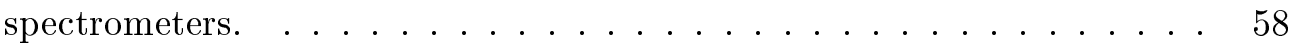

4.5. HDC offsets for Mainz. . . . . . . . . . . . . . . . . . . . . 59

4.6. Results of beam energy measurements during E93-049. . . . . . . . . . . 60

4.7. Corrected beam energy used for analyzing E93-049. . . . . . . . . . . 60

4.8. Electron beam polarization during E93-049 . . . . . . . . . . . . . . 61

4.9. Software cuts related to the acceptance of spectrometers at Mainz and JLab. . . . . . . . . . . . . . . . . . . 62

4.10. Software cuts on the electron momentum (in MeV) for JLab kinematics. 63

5.1. Software cuts on $z_{\text {close }} \ldots \ldots \ldots \ldots \ldots \ldots$. . . . . . . . . . . . .

5.2. Systematic uncertainties caused by spectrometer optics. . . . . . . . . 73

5.3. Systematic uncertainties in R caused by spin transport. . . . . . . . . . 74

6.1. Form factor ratio $\mu G_{E} / G_{M}$ for hydrogen. . . . . . . . . . . . 77

6.2. Limits on grid variables for phase space averaging. . . . . . . . . . . . 79

6.3. Super-ratio as function of missing momentum. . . . . . . . . . . . 80 


\section{List of Figures}

2.1. Feynman diagram for $p\left(e, e^{\prime} p\right) \ldots \ldots \ldots \ldots \ldots$

2.2. Breit frame ....................... 6

2.3. Kinematics and coordinate systems for $p\left(\vec{e}, e^{\prime} \vec{p}\right)$ scattering. . . . . . . . 8

2.4. Kinematics and coordinate systems for the $A\left(\vec{e}, e^{\prime} \vec{p}\right) B$ reaction. . . . . 9

2.5. Conceptual diagram for $A\left(\vec{e}, e^{\prime} \vec{p}\right) B$ as described by DWIA. . . . . . 14

2.6. Feynman diagrams representing the MEC and IC terms included in the $A\left(\vec{e}, e^{\prime} \vec{p}\right) B$ processes. . . . . . . . . . . . . . . . 17

2.7. The free and medium modified form factors $G_{E}$ and $G_{M}$ versus $Q^{2}$ in the color neutrality model. . . . . . . . . . . . . . . . . . 22

2.8. Medium effects on the form factor ratio $G_{E} / G_{M}$ vs. $Q^{2}$ as calculated in the $\mathrm{QMC}$ model. . . . . . . . . . . . . . . . . . . . . 23

3.1. Scheme of a racetrack microtron. . . . . . . . . . . . . . . 26

3.2. Floorplan of MAMI. . . . . . . . . . . . . . . . 27

3.3. Hall A1 at MAMI. . . . . . . . . . . . . . . . . . . . 28

3.4. Cryo-system of Hall A1 . . . . . . . . . . . . . . . . . . . . . . 30

3.5. Schematic view of target loop. . . . . . . . . . . . . . . . 31

3.6. Detector house of spectrometer A . . . . . . . . . . . . . . . . . 32

3.7. Working principle of a VDC. . . . . . . . . . . . . 33

3.8. Schematics of the HDC. . . . . . . . . . . . . . . . 35

3.9. Local circuit for the trigger detector system. . . . . . . . . . . . . . 36

3.10. Site plan of the CEBAF accelerator. . . . . . . . . . . . . . 38

3.11. Layout of Hall A. . . . . . . . . . . . . . . . . . . . . . 40

3.12. Schematic layout of the VDC. . . . . . . . . . . . . . 43

3.13. Detector package for the hadron arm . . . . . . . . . . . . . . . 43 
3.14. Coincidence trigger setup for Hall A. . . . . . . . . . . . . . . . 45

3.15. Hall A DAQ setup. . . . . . . . . . . . . . . . . . . . . . 46

4.1. Hall A laboratory coordinate system. . . . . . . . . . . . . . . . 48

4.2. Target coordinate system. . . . . . . . . . . . . . . . . . 49

4.3. Plot of $x_{\mathrm{BPMB}}^{\mathrm{lab}}$ versus $x_{\mathrm{rast}}^{\mathrm{lab}} \ldots \ldots \ldots \ldots \ldots \ldots \ldots$

4.4. Comparison of $z_{\text {close }}$ before and after FPP chamber alignment. . . . . . 57

4.5. Event distributions from elastic ep scattering used for pointing checks. . 58

4.6. Coincidence time cut. . . . . . . . . . . . . . . . . . . . 64

4.7. Missing mass distributions before and after the application of cuts. . . . 65

5.1. Schematic polarimeter. . . . . . . . . . . . . . 67

5.2. Example of an angular distribution of polarized protons scattered from

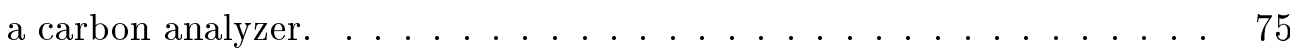

5.3. Analyzing power of the Hall A FPP measured in E94-049. . . . . . . . . 75

5.4. Schematic view of the spin precession in the magnetic field of a dipole. . 76

6.1. Experimental results for $\mu G_{E} / G_{M}$ in hydrogen elastic scattering. . . . . 78

6.2. Ratio $\mu G_{E} / G_{M}$ from experimental data compared with theoretical calculations. . . . . . . . . . . . . . . . . 79

6.3. Dependency of $R^{\exp }$ on current operator. . . . . . . . . . . . . . . 81

6.4. Dependency of $R^{\exp }$ on wave functions. . . . . . . . . . . 83

6.5. Comparison of measured $R^{\exp }$ with theoretical calculations. . . . . . . . 84

6.6. Super-Ratio $R^{\exp } / R^{\text {PWIA }}$ for all $Q^{2} \ldots \ldots \ldots \ldots$

6.7. In-medium to free proton F2 structure function as function of $x$ at threshold. . . . . . . . . . . . . . . . 86 


\section{Chapter 1}

\section{Introduction}

Electron scattering is a powerful tool to study nucleons and nuclei because the leptonic part of the interaction is well understood in terms of quantum electrodynamics. The electromagnetic coupling is weak and so allows the treatment of the reaction vertices to first order in the one-photon exchange approximation. A disadvantage of the relative weakness of the electromagnetic force is the small cross section, which requires comparatively long beam times and high luminosity on the experimental side. Recent developments in electron accelerator technology have made high luminosity, continuous wave, polarized electron beams available. In combination with the development of focal plane polarimeters, polarization observables became accessible experimentally.

The nucleon is an extended object, so the cross section for scattering of electrons from nucleons differs from the scattering from a point particle. This difference is described by form factors, which are determined by the spatial distribution of the nucleon. Two form factors are required to describe the nucleon. There are a number of different ways of expressing the form factors, but one set, the Sachs form factors, have a simple physical interpretation: one form factor describes the distribution of charge and the other the distribution of the magnetic moment. These form factors have been studied extensively by measuring cross sections in electron scattering experiments. Separation of the form factors requires measuring the cross section for the same momentum transfer but with different beam energies. At higher energies, i.e. small cross sections, the cross section is dominated by the magnetic form factor and separation of the two form factors becomes increasingly difficult.

The possibility of using polarization transfer measurements to extract form factors was first discussed by Akhiezer and Rekalo [1] and later by Arnold, Carlson and Gross 
[2]. For the reaction ${ }^{1} \mathrm{H}\left(\vec{e}, e^{\prime} \vec{p}\right)$, using a longitudinally polarized electron beam, the longitudinal $\left(P_{z}\right)$ and transverse $\left(P_{x}\right)$ transferred polarization of the proton can be measured. The ratio of these polarizations is proportional to the ratio of the electric to the magnetic Sachs form factor for the ${ }^{1} \mathrm{H}\left(\vec{e}, e^{\prime} \vec{p}\right)$ reaction. To measure the polarization ratio, highly polarized electron beams are required. Proton polarimeters have a small analyzing power, therefore high luminosities are needed to keep the experimental time in a reasonable limit. On the other hand, high luminosity pulsed beams would lead to a high fraction of accidental coincidences and thus introduce large uncertainties. The development of high luminosity, continuous wave accelerators with polarized electron sources have led to the option of doing polarization transfer ratio experiments. Recent experiments which used this technique [3] have achieved dramatic improvements in the precise measurement of the Sachs form factor ratio.

As has long been known, the nucleus can be described well with the assumption that nucleons retain their essential properties in the nucleus. The proximity of the nucleons in the nucleus, with an average spacing of little more than one diameter, and the strong force between them, leads naturally to the question of whether the nucleons modified in the nuclear medium. And more specifically, could there be changes in the charge and magnetic moment distributions? Answering this question has proven difficult and in principle there is no completely unambiguous answer. There are numerous effects due to the presence of other nucleons, such as meson exchange currents, and isobar configurations, and uncertainties in the description of the interaction between bound nucleons, such as the choice of the relativistic current operator and bound state wave function. Thus, any search for modifications of the form factors can only be done within the context of a particular model.

Polarization observables in the $A\left(\vec{e}, e^{\prime} \vec{p}\right) B$ reaction are sensitive to the electromagnetic properties of the bound nucleon. A change in the polarization transfer ratio for the bound nucleon compared to the free nucleon could give an indication of medium modifications. Compared to cross section measurements, polarization transfer provides a more sensitive test on the reaction dynamics. Therefore, measuring the polarization transfer ratio provides a powerful tool to probe the $\left(\vec{e}, e^{\prime} \vec{p}\right)$ reaction mechanism. The 
sensitivity of the method, providing constraints to the theoretical interpretation, makes it complementary to cross section measurements.

The goal of this work is to compare the ratio of the electric and magnetic Sachs form factors of the free proton to the same ratio for a bound proton. Clearly, even in a light but dense nucleus like helium several effects, e.g. isobar currents, meson exchange currents and two-body interactions, change the apparent nucleon form factors. The focus of this work is to look for medium modifications which occur in addition to these effects.

Possible changes of form factors have been investigated by previous experiments and upper limits have been found. Electron scattering experiments [4] measuring the Coulomb sum rule and the separation of the longitudinal and transverse response functions for a range of the four-momentum transfer squared, $Q^{2}$, between $0.1 \mathrm{GeV}^{2}$ and $0.5 \mathrm{GeV}^{2}$, determined the upper limit in the change of the electric form factor to be $10 \%$, and for the magnetic form factor $3 \%$. Measurements of $y$-scaling $[5,6]$ in the $\left(e, e^{\prime} p\right)$ reaction in the $Q^{2}$ range between $1 \mathrm{GeV}^{2}$ and $5 \mathrm{GeV}^{2}$ found the upper limit for a change in the electric form factor to be $10 \%$ and for the magnetic form factor to be $3 \%$.

This thesis describes the measurement of polarization transfer variables in the ${ }^{1} \mathrm{H}\left(\vec{e}, e^{\prime} \vec{p}\right)$ and ${ }^{4} \mathrm{He}\left(\vec{e}, e^{\prime} \vec{p}\right)^{3} \mathrm{H}$ reactions at five different kinematic settings. The measurement of the first kinematic setting was taken in Hall A1 at the MAMI accelerator facility in Mainz, Germany. The other four settings at higher four momentum transfers were taken in Hall A at JLab. These are the first measurements of polarization transfer in helium and the first measurements on a nucleus heavier than deuterium with a statistical uncertainty sufficient to draw significant conclusions on medium modifications.

The thesis is separated into seven chapters. In the second chapter, a summary of the theory of electron scattering including models and predictions relevant to this work will be given. Chapter Three will describe the experimental setup used in Mainz and at JLab. Chapter Four discusses the raw data analyses, followed by the extraction of polarization observables as described in Chapter Five. Chapter Six discusses the results and Chapter Seven gives a short conclusion and outlook for future experiments. 


\section{Chapter 2}

\section{Theory}

This chapter gives a short review of the theory of electron scattering on nucleons and nuclei relevant to the topic of this thesis; for a more complete discussion see e.g. Ref. [7]. Theoretical approaches used to interpret the experimental data of this work will be explained. This chapter will finish with an overview of nucleon models which predict a medium dependence of the nucleon structure and results from previous experiments investigating these aspects will be summarized.

\subsection{Electron Scattering}

\subsubsection{Electron-Nucleon Scattering}

Electron scattering from a nucleon or nucleus is described by QED. Figure 2.1 shows the Feynman diagram for the elastic ep reaction in the one photon approximation where a virtual photon couples to the electron and proton. The amplitude of the scattering process is given by the $T$-matrix and is the product of the electron and nucleon current

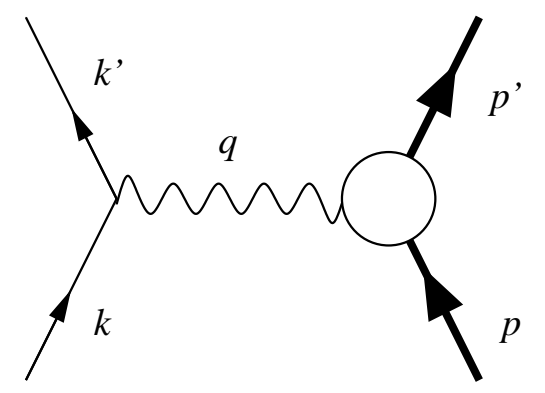

Figure 2.1: Lowest order Feynman diagram for the $p\left(e, e^{\prime} p\right)$ reaction. The electron with initial four momentum $k$ and final four momentum $k^{\prime}$ exchanges a virtual photon with four momentum $q$. The proton has initial and final four momenta of $p$ and $p^{\prime}$, respectively. 
matrix elements times the photon propagator $\left(1 / q^{2}\right)$ :

$$
T_{f i}=e^{2}\left\langle l\left(k^{\prime}\right)\left|j^{\mu}(0)\right| l(k)\right\rangle \frac{1}{q^{2}}\left\langle N\left(p^{\prime}\right)\left|J_{\mu}(0)\right| N(p)\right\rangle .
$$

The electron and hadron transition currents are denoted by $j^{\mu}$ and $J^{\mu}$, respectively. The cross section $\sigma$ is proportional to the squared $T$-matrix

$$
\sigma \approx\left|\bar{T}_{f i}\right|^{2}=\frac{e^{4}}{q^{4}} L_{\mu \nu} N^{\mu \nu}
$$

where $L_{\mu \nu}$ is the leptonic and $N^{\mu \nu}$ the nucleon or hadronic tensor. The electron transition current for the reaction is

$$
j^{\mu}(\vec{x})=-e \bar{u}\left(\vec{k}^{\prime}\right) \gamma^{\mu} u(\vec{k}) e^{i\left(\vec{k}^{\prime}-\vec{k}\right) \vec{x}}
$$

Here $u(\vec{k})$ and $u\left(\vec{k}^{\prime}\right)$ are the Dirac spinors for the incident and final electron with momentum $\vec{k}$ and $\vec{k}^{\prime}$, and $\gamma^{\mu}$ are the Dirac matrices. Since the proton is a composite system with internal structure, the proton transition current is more complex than the electron transition current. To obtain the proton transition current, the $\gamma^{\mu}$-matrix in Eq. 2.3 is replaced by the most general Lorentz four-vector. Upon spin averaging, the requirement of Lorentz-invariance, conservation of the electromagnetic current and parity conservation (neglecting the weak force) leads to a linear combination of $\gamma^{\mu}$ and $i \sigma^{\mu \nu} q_{\nu}$ as one choice for the propagator. Therefore, the proton transition current can be written as

$$
J^{\mu}=e \bar{u}\left(\vec{p}^{\prime}\right)\left[F_{1}\left(Q^{2}\right) \gamma^{\mu}+\frac{1}{2 m_{p}} F_{2}\left(Q^{2}\right) i \sigma^{\mu \nu} q_{\nu}\right] u(\vec{p}) e^{i\left(\vec{p}^{\prime}-\vec{p}\right) \cdot \vec{x}},
$$

where $F_{1}$ and $F_{2}$ are the independent Pauli and Dirac form factors of the proton and $m_{p}$ its mass. The four momentum transfer squared, $Q^{2}$, is related to $q$ through $Q^{2}=-q^{2}$. The form factor contains the information about the spatial distribution of the electric charge and magnetic moment. In the simplest case, using the Born approximation and neglecting the recoil momentum, the form factor is the Fourier transform of the charge (magnetic moment) distribution. The normalization of these form factors at the photon point, $Q^{2}=0$, is

$$
\begin{aligned}
& F_{1}(0)=1, \\
& F_{2}(0)=\kappa_{p} .
\end{aligned}
$$




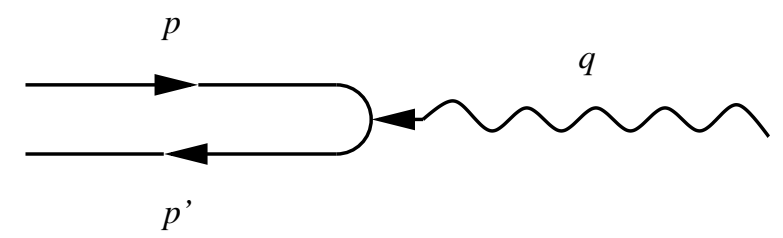

Figure 2.2: Definition of the Breit or brick-wall frame. In this reference frame $\vec{p}=-\vec{p}^{\prime}$, therefore no energy is transferred by the virtual photon $(\omega=0)$.

with the anomalous magnetic moment of the proton $\kappa_{p}$. The resulting differential cross section can be shown [8] to have the form:

$$
\frac{d \sigma}{d \Omega}=f_{\mathrm{rec}}\left(\frac{d \sigma}{d \Omega}\right)_{\mathrm{Mott}}\left[F_{1}^{2}+\frac{Q^{2}}{4 m_{p}^{2}}\left(2\left(F_{1}+F_{2}\right)^{2} \tan ^{2}\left(\frac{\theta_{e}}{2}\right)+F_{2}^{2}\right)\right] .
$$

with $\theta_{e}$ the laboratory frame electron scattering angle. The recoil factor $f_{\text {rec }}$ is the ratio of the final to initial electron energy $E_{e}^{\prime} / E_{e}$ and is given by

$$
f_{\text {rec }}=\left[1+\frac{2 E_{e}}{m_{p}} \sin ^{2}\left(\frac{\theta_{e}}{2}\right)\right]^{-1}
$$

The Mott cross section,

$$
\left(\frac{d \sigma}{d \Omega}\right)_{\text {Mott }}=\frac{(Z \alpha)^{2} \cos ^{2}\left(\frac{\theta_{e}}{2}\right)}{4 E_{e}^{2} \sin ^{4}\left(\frac{\theta_{e}}{2}\right)},
$$

describes elastic scattering of an electron on a heavy, pointlike and spinless particle with a charge of $Z e ; \alpha=\frac{e^{2}}{\hbar c}$ is the fine structure constant.

It is convenient to use a different parametrization of the form factors. The Sachs electric and magnetic form factors $G_{E}^{p}$ and $G_{M}^{p}[9]$ are a linear combination of $F_{1}$ and $F_{2}$,

$$
\begin{aligned}
G_{E}^{p} & =F_{1}-\tau F_{2} \\
G_{M}^{p} & =F_{1}+F_{2},
\end{aligned}
$$

where $\tau=Q^{2} / 4 m_{p}^{2}$. The electric and magnetic form factors of the neutron are defined similarly. In the Breit frame, illustrated in Fig. 2.2, these form factors are closely related to the proton charge and magnetic moment distributions. More specifically, $G_{E}$ is linked to the scalar part of the electromagnetic current, while $G_{M}$ comes from the vector part:

$$
\begin{aligned}
\left\langle N_{s^{\prime}}(\vec{q} / 2)\left|J^{0}(0)\right| N_{s}(-\vec{q} / 2)\right\rangle & =2 M_{p} G_{E}\left(\vec{q}^{2}\right) \delta_{s^{\prime} s} \\
\left\langle N_{s^{\prime}}(\vec{q} / 2)|\vec{J}(0)| N_{s}(-\vec{q} / 2)\right\rangle & =2 G_{M}\left(\vec{q}^{2}\right) \chi_{s^{\prime}}^{\dagger} i \vec{\sigma} \times \vec{q} \chi_{s}
\end{aligned}
$$


For $Q^{2}=0$ the Sachs proton form factors are:

$$
\begin{aligned}
G_{E}^{p}(0) & =1 \\
G_{M}^{p}(0) & =\mu_{p},
\end{aligned}
$$

where $\mu_{p}$ is the magnetic moment of the proton. In the following, the discussion will be limited to proton form factors. Therefore, the superscripts $p$ will be omitted, so that $G_{E} \equiv G_{E}^{p}$ and $G_{M} \equiv G_{M}^{p}$. Rewriting Eq. (2.7) in terms of the Sachs form factors, the cross section becomes:

$$
\frac{d \sigma}{d \Omega}=f_{\mathrm{rec}}\left(\frac{d \sigma}{d \Omega}\right)_{\mathrm{Mott}} \frac{1}{1+\tau}\left(G_{E}^{2}+\frac{\tau}{\epsilon} G_{M}^{2}\right)
$$

with $\epsilon=\left[1+2(1+\tau) \tan ^{2}(\theta / 2)\right]^{-1}$. This permits a simple determination of $G_{E}^{2}$ and $G_{M}^{2}$ using a Rosenbluth diagram [10] in which cross section data at a given momentum transfer $Q^{2}$ are plotted against $\tan ^{2} \frac{\theta_{e}}{2}$ :

$$
\frac{d \sigma / d \Omega}{(d \sigma / d \Omega)_{\mathrm{Mott}}}=A\left(Q^{2}\right)+B\left(Q^{2}\right) \tan ^{2} \frac{\theta_{e}}{2}
$$

For high $Q^{2}$ the extraction of $G_{E}$ is difficult since the cross section is dominated by $G_{M}$. The $G_{M}$ dominance at large $Q^{2}$ is related to the fact that the magnetic force $\left(\sim 1 / r^{3}\right)$ dominates at short distances relative to the electric force $\left(\sim 1 / r^{2}\right)$. The Rosenbluth separation in this energy range requires very precise knowledge of the experimental cross section and thus the extraction of $G_{E}$ is very sensitive to experimental uncertainties. In [1] and [2] it was pointed out that measuring polarization transfer in $e p$ gives access to the ratio $G_{E} / G_{M}$. Therefore, the combination of Rosenbluth data, providing $G_{M}$, with polarization transfer measurements, providing $G_{E} / G_{M}$, can be used to extract $G_{E}$ at higher $Q^{2}[11]$.

Figure (2.3) shows the kinematics and coordinate systems for ${ }^{1} \mathrm{H}\left(\vec{e}, e^{\prime} \vec{p}\right)$ in the laboratory system. The longitudinally polarized electron exchanges a virtual photon with the proton. In elastic scattering, the proton momentum is parallel to the momentum of the virtual photon. The unit vectors of the $(x, y, z)$-coordinate system are defined as:

$$
\hat{z}=\frac{\vec{q}}{|\vec{q}|}, \quad \hat{y}=\frac{\vec{k} \times \vec{k}^{\prime}}{\left|\vec{k} \times \vec{k}^{\prime}\right|} \quad \text { and } \quad \hat{x}=\hat{y} \times \hat{z} .
$$




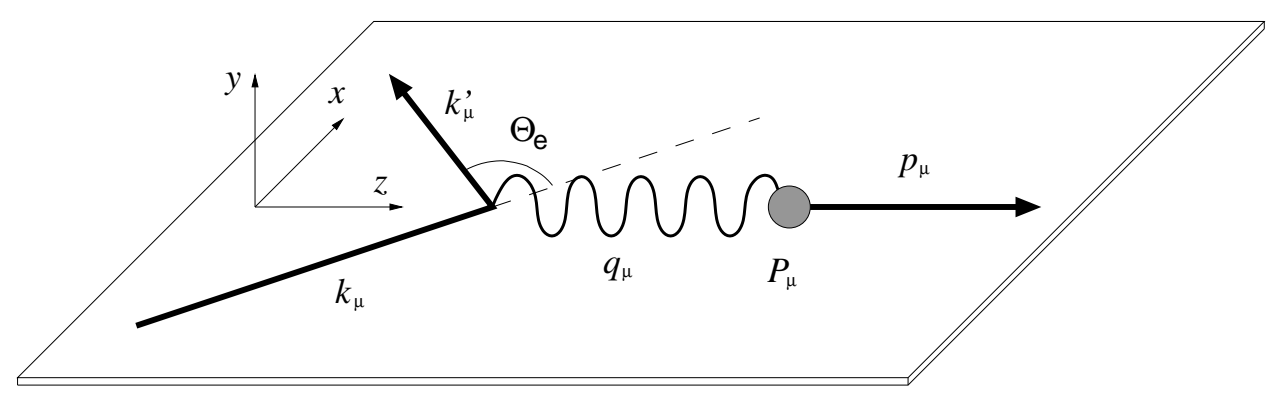

Figure 2.3: Kinematics and coordinate systems for $p\left(\vec{e}, e^{\prime} \vec{p}\right)$ scattering.

For $e p$ scattering, as for any other two-body-to-two-body processes, parity invariance forbids induced polarization in the scattering plane, $P_{x}=P_{z}=0$, and transferred polarization from the scattering plane to polarization perpendicular to the scattering plane, $P_{y}^{\prime}=0$ [12]. For pure one-photon exchange, $P_{y}$ is also zero. The nonzero polarizations are

$$
\begin{aligned}
& I_{0} P_{x}^{\prime}=\frac{E_{e}+E_{e}^{\prime}}{m_{p}} \sqrt{\tau(1+\tau)} G_{M}^{2} \tan \left(\frac{\theta_{e}}{2}\right) \\
& I_{0} P_{z}^{\prime}=-2 \sqrt{\tau(1+\tau)} G_{E} G_{M} \tan ^{2}\left(\frac{\theta_{e}}{2}\right)
\end{aligned}
$$

where $I_{0}$ is defined as

$$
I_{0}=G_{E}^{2}+\frac{\tau}{\epsilon} G_{M}^{2}
$$

From Eqs. (2.19) and (2.21) the ratio of the electric to the magnetic form factor can be easily determined:

$$
\frac{G_{E}}{G_{M}}=-\frac{P_{x}^{\prime}}{P_{z}^{\prime}} \frac{E_{e}+E_{e}^{\prime}}{2 m_{p}} \tan \left(\frac{\theta_{e}}{2}\right)
$$

The ratio of form factors is equal to the ratio of transferred polarization components times a known kinematic factor.

\subsubsection{Electron-Nucleus Scattering}

The vertex for electron-nucleus scattering is more complicated than the electronnucleon scattering vertex. Figure 2.4 shows the kinematics for the $A\left(\vec{e}, e^{\prime} \vec{p}\right) B$ reaction in the laboratory system. While the electron scattering plane is similar to Fig. 2.3, the hadron reaction plane can now be tilted by an angle $\Phi_{x}$. The momentum of the knockout proton with four-vector $p_{\mu}=\left(E_{p}, \vec{p}_{p}\right)$ need no longer be parallel to the momentum 


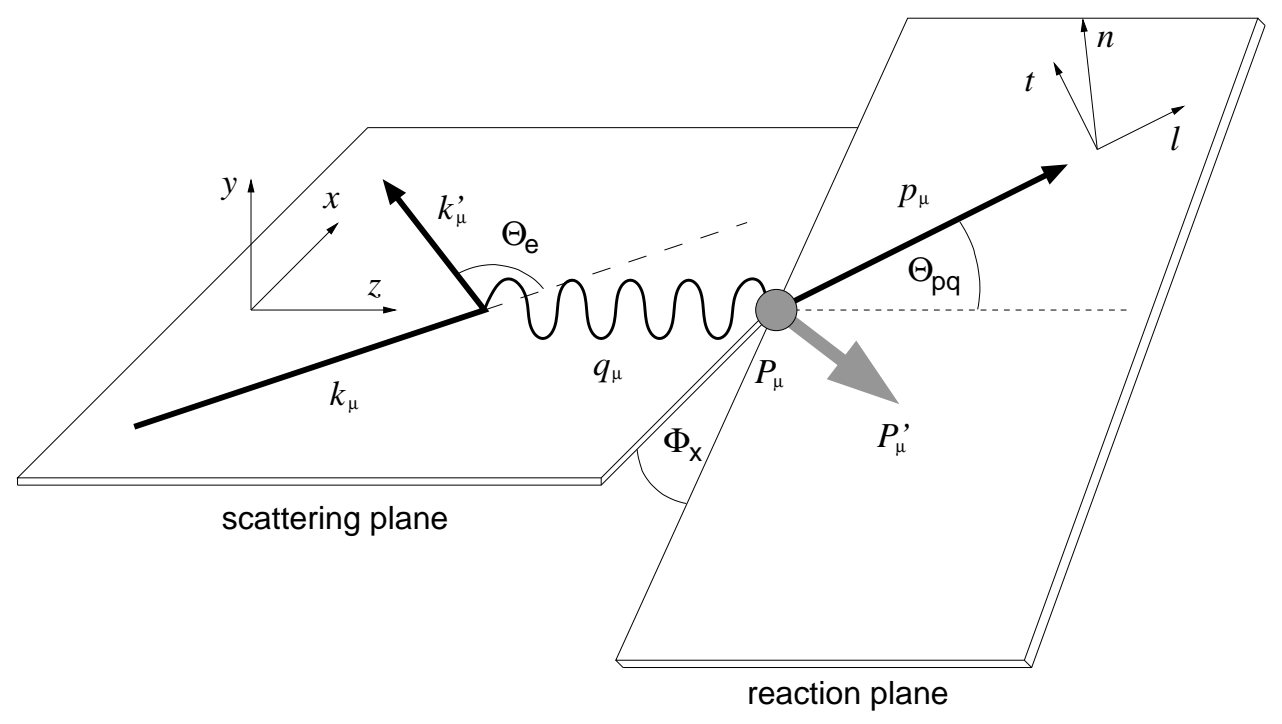

Figure 2.4: Kinematics and coordinate systems for the $A\left(\vec{e}, e^{\prime} \vec{p}\right) B$ reaction.

transfer axis. The recoiling nucleus with mass $M$ carries away the four-momentum $P_{\mu}^{\prime}=\left(E_{r}, \vec{p}_{r}\right)$. The $n l t-$ coordinate system associated with the hadron scattering plane is defined in the hadron scattering plane:

$$
\hat{l}=\frac{\vec{p}_{p}}{\left|\vec{p}_{p}\right|}, \quad \hat{n}=\frac{\vec{p}_{p} \times \vec{p}_{r}}{\left|\vec{p}_{p} \times \vec{p}_{r}\right|} \quad \text { and } \quad \hat{t}=\hat{n} \times \hat{l} .
$$

The helicity of the electron beam is denoted by $h$, the projections of the proton spin $\vec{s}$ of the unit vectors $\hat{n}, \hat{l}$, and $\hat{t}$ are defined as

$$
\mathcal{S}_{n}=\hat{n} \cdot \vec{s}, \quad \mathcal{S}_{l}=\hat{l} \cdot \vec{s} \quad \text { and } \quad \mathcal{S}_{t}=\hat{t} \cdot \vec{s}
$$

It can be shown that for a discrete final state of the recoiling nucleus [7] the semiinclusive cross section can be written as

$$
d \sigma=(2 \pi)^{-3} \frac{E_{e}^{\prime}}{E_{e}} \frac{\alpha}{Q^{2}} \nu_{\mu \nu} \mathcal{W}^{\mu \nu} d E_{e}^{\prime} d \Omega_{e} d^{3} p_{p}
$$

where $\nu_{\mu \nu}$ is the electron response tensor, $\mathcal{W}^{\mu \nu}$ the nuclear response tensor and $d \Omega_{e}$ the solid angle for the electron momentum in the laboratory frame. For unpolarized electron scattering, further analysis reveals that in the tensor product $\nu_{\mu \nu} \mathcal{W}^{\mu \nu}$ the kinematic and dynamic dependencies can be separated:

$$
\nu_{\mu \nu} \mathcal{W}^{\mu \nu}=4 E_{e} E_{e}^{\prime} \cos ^{2}\left(\frac{\theta_{e}}{2}\right)\left[V_{L} R_{L}+V_{T} R_{T}+V_{L T} R_{L T} \cos \phi_{x}+V_{T T} R_{T T} \cos 2 \Phi_{x}\right]
$$


The kinematic factors are

$$
\begin{aligned}
V_{L} & =\frac{Q^{4}}{q^{4}} \\
V_{T} & =\frac{Q^{2}}{2 q^{2}}+\tan ^{2}\left(\frac{\theta_{e}}{2}\right) \\
V_{L T} & =\frac{Q^{2}}{q^{2}}\left[\frac{Q^{2}}{q^{2}}+\tan ^{2}\left(\frac{\theta_{e}}{2}\right)\right]^{\frac{1}{2}} \\
V_{T T} & =\frac{Q^{2}}{2 q^{2}}
\end{aligned}
$$

and the four independent response functions which describe the electromagnetic properties of the hadronic system are $R_{L}, R_{T}, R_{L T}$, and $R_{T T}$. The resulting inclusive electron scattering cross section

$$
\frac{d \sigma}{d \Omega}=f_{\mathrm{rec}}\left(\frac{d \sigma}{d \Omega}\right)_{\mathrm{Mott}}\left[V_{L} R_{L}+V_{T} R_{T}\right]
$$

links the unpolarized response functions with the Sachs electric and magnetic form factors as given in Eq. 2.16.

For studying nucleon knockout reactions of the type $A\left(\vec{e}, e^{\prime} \vec{p}\right) B$, where a polarized beam is used and the polarization of the ejectile nucleon is measured, additional response functions are required. Omitting the derivation (given e.g. in $[7,13]$ ), the differential cross section is

$$
\begin{aligned}
\left(\frac{d^{3} \sigma}{d E_{e}^{\prime} d \Omega_{e^{\prime}} d \Omega_{p}}\right)= & \frac{m_{p}\left|\vec{p}_{p}\right|}{2(2 \pi)^{3}}\left(\frac{d \sigma}{d \Omega}\right)_{\mathrm{Mott}} \\
& \left\{V_{L}\left(R_{L}+R_{L}^{n} \mathcal{S}_{n}\right)+V_{T}\left(R_{T}+R_{T}^{n} \mathcal{S}_{n}\right)\right. \\
& +V_{T T}\left[\left(R_{T T}+R_{T T}^{n} \mathcal{S}_{n}\right) \cos 2 \Phi_{x}+\left(R_{T T^{\prime}}^{l} \mathcal{S}_{l}+R_{T T^{\prime}}^{t} \mathcal{S}_{t}\right) \sin 2 \Phi_{x}\right] \\
& +V_{L T}\left[\left(R_{L T}+R_{L T}^{n} \mathcal{S}_{n}\right) \cos \Phi_{x}+\left(R_{L T} \mathcal{S}_{l}+R_{L T}^{t} \mathcal{S}_{t}\right) \sin \Phi_{x}\right] \\
& +h V_{L T^{\prime}}\left[\left(R_{L T^{\prime}}+R_{L T^{\prime}}^{n} \mathcal{S}_{n}\right) \sin \Phi_{x}+\left(R_{L T^{\prime}}^{l} \mathcal{S}_{l}+R_{L T^{\prime}}^{t} \mathcal{S}_{t}\right) \cos \Phi_{x}\right] \\
& \left.+h V_{T T^{\prime}}\left[R_{T T^{\prime}}^{l} \mathcal{S}_{l}+R_{T T^{\prime}}^{t} \mathcal{S}_{t}\right]\right\}
\end{aligned}
$$

The additional kinematic factors, which are correlated to the antisymmetric part of the electron and nucleon tensors, are defined as

$$
\begin{aligned}
V_{L T^{\prime}} & =\frac{Q^{2}}{q^{2}} \tan \left(\frac{\theta_{e}}{2}\right) \\
V_{T T^{\prime}} & =\tan \left(\frac{\theta_{e}}{2}\right)\left[\frac{Q^{2}}{q^{2}}+\tan \left(\frac{\theta_{e}}{2}\right)\right]^{\frac{1}{2}} .
\end{aligned}
$$


It is useful to study the properties of the 18 response functions for parallel kinematics and for the plane wave impulse approximation (PWIA). In parallel kinematics, where $\vec{q} \| \vec{p}_{p}$, the angle $\Phi_{p}$ becomes undefined. Therefore, all response functions whose contributions to the observables retain explicit dependencies of $\Phi_{p}$ must vanish. In addition, the transverse polarization has to be independent of $\Phi_{p}$ because the orientation of the reaction plane becomes undefined. A short calculation leads to the constraints

$$
\begin{aligned}
& R_{L T}^{n}=+R_{L T}^{s} \\
& R_{L T}^{\prime n}=-R_{L T}^{\prime s} .
\end{aligned}
$$

For the PWIA limit the operators and states have to be symmetric with respect to the operation of parity and time reversal, $\Theta=\mathcal{P} \mathcal{T}$. Omitting the derivations given in $[13-15]$, we note that the nuclear response tensor $\mathcal{W}$ must be symmetric and hermitic, hence real in the plane wave limit. In addition, $\mathcal{W}^{\mu \nu}$ has to be symmetric with respect to exchange of its indices combined with inversion of spins. Therefore, the symmetric part of the nuclear response tensor $\mathcal{W}_{S}^{\mu \nu}$ does not contribute to the recoil polarization, while the antisymmetric part $\mathcal{W}_{A}^{\mu \nu}$ only contributes to the spin dependent part of the cross section. An overview of response function properties is given in Table 2.1.

\subsection{Theoretical Models and Predictions}

Starting from the most general cross section for electron scattering, several assumptions simplify the task of theoretically describing the $\left(e, e^{\prime} N\right)$ reaction. The most simplified approach is the PWIA, where an undisturbed electron wave interacts with a nucleus, and the knockout nucleon does not interact with the residual nucleus. The distorted wave impulse approximation (DWIA) is a refinement of the basic PWIA taking into account final state interactions of the outgoing nucleon and the Coulomb distortion of the incident and scattered electron. In the following the plane wave impulse approximation (PWIA) and the relativistic distorted wave impulse approximation (RDWIA) will be discussed with special focus on their application in the three theory codes used in this work: LEA [16] written by J. J. Kelly, a code from the Madrid group of J. M. Udias [17] (both RDWIA codes) and the calculation by J.-M. Laget [18]. 


\begin{tabular}{cccccc}
\hline \hline response & helicity & & survives & & $\begin{array}{c}\text { azimuthal } \\
\text { function }\end{array}$ \\
dependence & in plane & parallel & PWIA & dependence \\
\hline$R_{L}$ & $\times$ & $\sqrt{ }$ & $\sqrt{ }$ & $\sqrt{ }$ & 1 \\
$R_{T}$ & $\times$ & $\sqrt{ }$ & $\sqrt{ }$ & $\sqrt{ }$ & 1 \\
$R_{L T}$ & $\times$ & $\sqrt{ }$ & $\times$ & $\sqrt{ }$ & $\cos \Phi_{x}$ \\
$R_{T T}$ & $\times$ & $\sqrt{ }$ & $\times$ & $\sqrt{ }$ & $\cos 2 \Phi_{x}$ \\
$R_{L T}^{\prime}$ & $\sqrt{ }$ & $\times$ & $\times$ & $\times$ & $\sin \Phi_{x}$ \\
\hline$R_{L}^{n}$ & $\times$ & $\sqrt{ }$ & $\times$ & $\times$ & 1 \\
$R_{T}^{n}$ & $\times$ & $\sqrt{ }$ & $\times$ & $\times$ & 1 \\
$R_{L T}^{n}$ & $\times$ & $\sqrt{ }$ & $\sqrt{ }$ & $\times$ & $\cos \Phi_{x}$ \\
$R_{T T}^{n}$ & $\times$ & $\sqrt{ }$ & $\times$ & $\times$ & $\cos 2 \Phi_{x}$ \\
$R_{L T}^{\prime n}$ & $\sqrt{ }$ & $\times$ & $\mp R_{L T}^{\prime S}$ & $\sqrt{ }$ & $\sin \Phi_{x}$ \\
\hline$R_{L T}^{l}$ & $\times$ & $\times$ & $\times$ & $\times$ & $\sin \Phi_{x}$ \\
$R_{T T}^{\prime l}$ & $\sqrt{ }$ & $\sqrt{ }$ & $\times$ & $\sqrt{ }$ & $\cos \Phi_{x}$ \\
$R_{T T}^{l}$ & $\times$ & $\times$ & $\times$ & $\times$ & $\sin 2 \Phi_{x}$ \\
$R_{T T}^{\prime l}$ & $\sqrt{ }$ & $\sqrt{ }$ & $\sqrt{ }$ & $\sqrt{ }$ & 1 \\
\hline$R_{L T}^{t}$ & $\times$ & $\times$ & $\pm R_{L T}^{N}$ & $\times$ & $\sin \Phi_{x}$ \\
$R_{L T}^{\prime t}$ & $\sqrt{ }$ & $\sqrt{ }$ & $\sqrt{ }$ & $\sqrt{ }$ & $\cos \Phi_{x}$ \\
$R_{T T}^{t}$ & $\times$ & $\times$ & $\times$ & $\times$ & $\sin 2 \Phi_{x}$ \\
$R_{T T}^{\prime t}$ & $\sqrt{ }$ & $\sqrt{ }$ & $\times$ & $\sqrt{ }$ & 1 \\
\hline \hline & & & & & \\
\hline
\end{tabular}

Table 2.1: Properties of response functions for the $A\left(\vec{e}, e^{\prime} \vec{N}\right) B$ reaction. The constraints among response functions for parallel/antiparallel kinematics are indicated by the upper/lower choices of signs. Table taken from [7].

\subsubsection{PWIA}

In PWIA some simplifying assumptions are made. The first assumption is generally the plane wave Born approximation (PWBA), which means that the electron scattering process is treated to first order perturbation only. It implies that for the extreme relativistic limit, where $E_{e} \gg m_{e}$, there is only one photon exchanged between the scattered electron and the target nucleus. Studies of Coulomb distortions [19] confirm that for intermediate energy electron scattering PWBA is a very good approximation. The second assumption is that the initial and final states can be represented as products of plane waves for the nucleon and the residual nucleus. This means the residual nucleus 
$\mathrm{B}$ in the $A\left(\vec{e}, e^{\prime} \vec{p}\right) B$ reaction is treated as spectator which implies that there are no final state interactions (FSI) between the knockout nucleon and the residual nucleus. A third assumption is that the nucleon which absorbs the photon is detected, i.e., exchange terms are ignored. Then the total nuclear response is equal to the sum of individual nucleon responses, so the scattering is treated as if free nucleons are involved; there are no two or three body forces included. When all these assumptions are applied in nonrelativistic PWIA, the knockout cross section factorizes in the form

$$
\frac{d \sigma}{d E_{e}^{\prime} d \Omega_{e} d E_{p} d \Omega_{p}}=K \sigma_{e p} S\left(E_{m}, \vec{p}_{m}\right)
$$

where $K$ is a kinematic factor correcting for the phase space, $\sigma_{e p}$ a term depending on the off-shell nucleon current, and $S\left(E_{m}, \vec{p}_{m}\right)$, called the spectral function, contains the nuclear structure information. Throughout this work, we have used the conventions given in [20] for the nucleon current. Summarizing the results it can be seen that the polarization process in PWIA is essentially the result of a transfer of the intrinsic angular momentum (helicity) of the virtual photon to the target nucleon.

\subsubsection{DWIA and RDWIA}

In DWIA, the rescattering of the ejectile nucleon from the residual nucleus after the electron-nucleon interaction is taken into account. A conceptual diagram is shown in Fig. 2.5. The one-photon exchange amplitude can be expressed in the form:

$$
M_{f i}=\int \frac{d^{3} \vec{q}}{(2 \pi)^{3}} J_{\mu}^{e}(\vec{q}) \frac{1}{Q^{2}} J_{N}^{\mu}(\omega, \vec{q})
$$

where $J_{e}^{\mu}$ is the electron current, $J_{N}^{\mu}$ the nucleon current and $\omega$ the energy of the virtual photon. In RDWIA, the nucleon current

$$
J_{N}^{\mu}(\omega, \vec{q})=\int d \vec{p} \bar{\Psi}_{F}(\vec{p}+\vec{q}) \hat{J}_{N}^{\mu}(\omega, \vec{q}) \Psi_{B}(\vec{p})
$$

is calculated with relativistic wave functions,$\Psi_{B}$ and $\Psi_{F}$, for initial bound and final outgoing nucleons. 


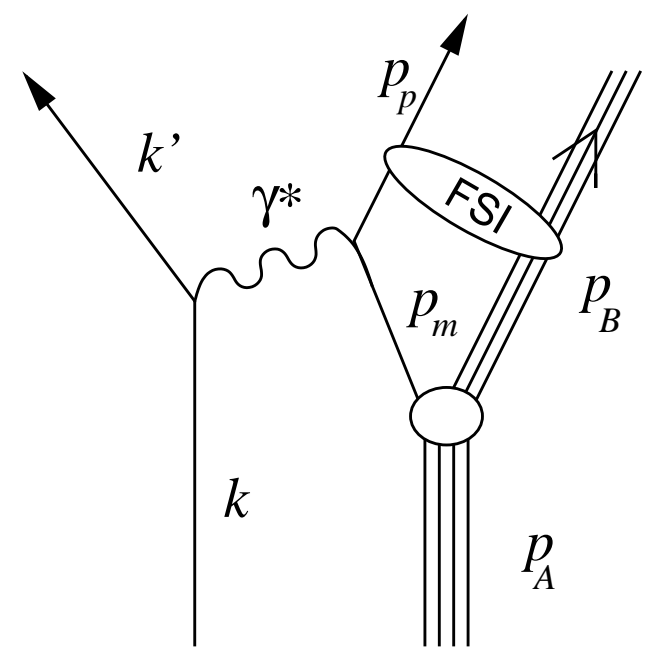

Figure 2.5: Conceptual diagram for $A\left(\vec{e}, e^{\prime} \vec{p}\right) B$ as described by DWIA.

\section{Bound State Wave Function}

Customarily bound state wave functions are either solutions of the Dirac equation with phenomenological Woods-Saxon potential wells or Dirac-Hartree solutions from a relativistic Lagrangian with scalar and vector (SV) meson terms [21].

In linear expansion analysis (LEA), the following approach was taken to get relativized wave functions. Starting from a nonrelativistic formalism based on bi-spinor solutions of a Schrödinger-like equation, one can construct normalized four-spinors to be used in Eq. 2.39 of the form

$$
\Psi_{\mathrm{NR}}=\frac{1}{\sqrt{N}}\left(\chi(\vec{p}), \frac{\vec{\sigma} \cdot \vec{p}}{E+m_{p}} \chi(\vec{p})\right)
$$

Then the calculation relativized by rewriting the Dirac equation for the upper component as a Schrödinger-like equation and introducing its nonrelativistic bispinor $\chi$ in Eq. 2.40. Comparing this solution to the upper component of the fully relativistic solution, an additional factor $i$ has to be introduced. One can then build a nonrelativistic formalism with central and spin-orbit potentials equivalent to the relativistic potentials and incorporating the Darwin term in order to recover the same upper component as in RDWIA. Udias uses the fully relativistic approach in his model. An additional relativistic effect is the enhancement of the lower components of the fully relativistic 
solution in the nuclear interior:

$$
\chi(\vec{p})_{\text {lower }}=\frac{\vec{\sigma} \cdot \vec{p}}{E+m_{p}+S-V} \chi(\vec{p})_{\text {higher }},
$$

where $S(V)$ is a scalar (vector) potential. This enhancement of the lower components with regard to free spinors is called spinor distortion.

\section{Electron Distortion, EMA}

In the absence of Coulomb distortion, the nuclear current could be evaluated for a unique value of the momentum transfer obtained from asymptotic electron kinematics. For light nuclei, even in the presence of Coulomb distortion the electron current is sharply peaked about the effective momentum transfer $\mathbf{q}^{\prime} \approx \mathbf{q}_{\text {eff }}$. The vertex function can then be evaluated in the effective momentum approximation (EMA). The lower components in Eq. 2.40 are given by

$$
\chi(\vec{p})_{\text {lower }}=\frac{\vec{\sigma} \cdot \vec{p}}{E+m_{p}} \chi(\vec{p})_{\text {upper }} \approx \vec{\sigma} \cdot \vec{p}_{\text {as }},
$$

with $p_{\text {as }}$ the momentum corresponding to the asymptotic kinematics at the nucleon vertex. A good discussion of the effects of EMA and spinor distortions can be found in [22].

\section{Current Operators}

For the relativistic nucleon current operator $\hat{J}_{N}^{\mu}(\omega, \vec{q})$ the definitions given in [20] were used:

$$
\begin{aligned}
\hat{J}_{C C 1}^{\mu} & =\left(F_{1}+F_{2}\right) \gamma^{\mu}-\frac{F_{2}}{2 m_{p}}\left(P^{\prime}+p_{m}\right)^{\mu} \\
\hat{J}_{C C 2}^{\mu} & =F_{1} \gamma^{\mu}+i \frac{\sigma^{\mu \nu} q_{\nu}}{2 m_{p}} F_{2}
\end{aligned}
$$

The four momentum transfer is $q_{\nu}=(\omega, \vec{q})$ at the electron vertex, the missing four momentum $p_{m}=\left(\bar{E}, \vec{p}_{m}\right), \vec{p}_{m}=\vec{p}_{p}-\vec{q}, \bar{E}=\sqrt{\vec{p}_{p}^{2}+m_{p}^{2}}$ (the final nucleon is asymptotically on-shell), and $\bar{E}=\sqrt{\vec{p}_{m}^{2}+m_{p}^{2}}$. Note that the $\omega$ used for $\hat{J}_{C C 1}^{\mu}$ is different from $\bar{\omega}=E^{\prime}-\bar{E}$ as implied in $\hat{J}_{C C 2}^{\mu}$. This means for extrapolating these operators off-shell free spinors for the momentum $\vec{p}$ and mass $m_{p}$ are employed, but the energy 
$E$ used within the vertex function is replaced by the value $\bar{E}$ it would have had if the nucleon were on-shell in the initial state. Both current operators give identical results for elastic scattering from a moving but free nucleon. They are also equivalent on-shell by virtue of the Gordon identity.

\section{Optical Potentials}

The outgoing nucleon scattering wave function is a solution of the Dirac equation containing SV optical potentials. The optical potentials are usually obtained by fitting proton elastic scattering data using Woods-Saxon shapes. There are two approaches to handling the energy dependence of the parameters. One is to use single energy fits, but parameters may vary erratically due to correlations. The second uses a broad range of proton energies, which can have adverse effects on the local fit quality. In general, optical potentials do usually not extrapolate well in mass and energy. In this work, we studied the sensitivity of our theoretical results to various optical potentials. Details of the potentials used will be discussed in Chap. 6 .

\section{MEC and IC (Ryckebusch)}

While independent particle models like PWIA and RDWIA can be used to calculate the momentum distributions relatively well, they fail at momenta below the Fermi momentum. The reason is probably that at these momenta two-body currents and subnuclear degrees of freedom have to be taken into account. "Unfortunately, for finite nuclei a relativistic, empirically well-founded and practicable relativistic nuclear-structure model that also accounts for the multinucleon degrees of freedom is not yet available." (J. Ryckebusch, in [23]). An answer to the question of how much the meson exchange currents (MEC) and isobar currents (IC) can only be given by studying the relative importance of these effects in a nonrelativistic model, as e.g. provided by Ryckebusch and his group. There, the two-body currents are treated in their full nonrelativistic complexity. The bound and scattering states are calculated using the Hartree-Fock method using a Skyrme nucleon-nucleon force. The current operators and distorted outgoing nucleon wave are treated by a multipole expansion. The one-body current is 


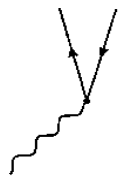

(a)

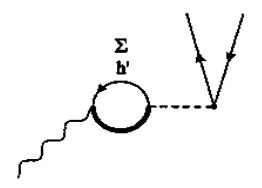

(e)

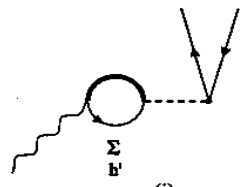

(i)

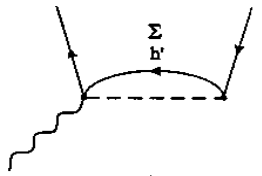

(b)

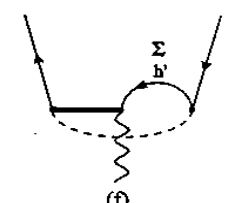

(f)

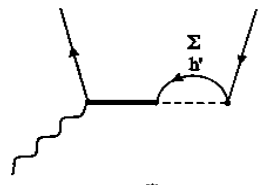

(j)

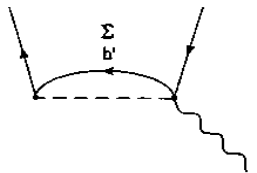

(c)

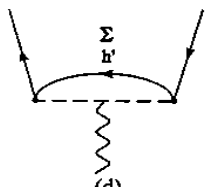

(d)

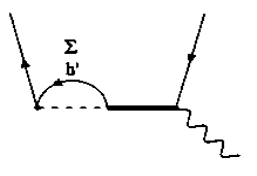

(g)

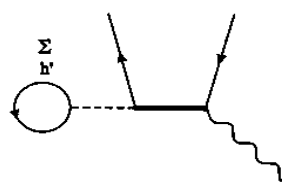

(h)

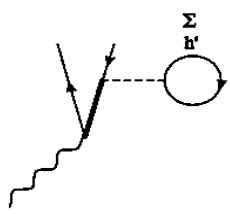

(1)

Figure 2.6: Feynman diagrams representing the MEC and IC terms included in the $A\left(\vec{e}, e^{\prime} \vec{p}\right) B$ processes. Wavy, thin, thick, and dashed lines denote photons, nucleons, $\Delta$ 's, and pions. Nucleon lines with an arrow down (up) refer to occupied (scattering) states. Diagram (a) refers to conventional impulse approximation. Diagrams (b) - (d) are related to MEC, whereas (e) - (l) represent the different and indirect IC contributions. Figure taken from [23].

derived from the on-shell covariant nucleon current. One has to note, though, that for the nonrelativistic reduction, the standard Foldy-Wouthuysen and the "direct Pauli" approach lead to different results. In the work of Ryckebusch, the Foldy-Wouthuysen method is used. In the charge density operator, spin-orbit terms are included.

For the MEC, the pion is the predominant meson. The MEC terms related to pion exchange are derived from the one-pion exchange potential. Diagrams (b)-(d) in Fig. 2.6 show a representation of the reaction processes connected to MEC. The IC, which are dominated by the $\Delta_{33}$, cannot be constrained through charge-current conservation. They are therefore called "model dependent" and also show strong medium dependences. As shown in Fig. 2.6, all isobars are attached to the photon line. Theoretically, MEC for quasi-elastic ${ }^{4} \mathrm{He}\left(\vec{e}, e^{\prime} \vec{p}\right)^{3} \mathrm{H}$ scattering are small. In general, MEC and IC tend to work in opposite direction and cancel each other partially. 


\subsubsection{Two-Body Currents}

Laget's method [24] to calculate electrodisintegration of ${ }^{4} \mathrm{He}$ is an extension of the diagrammatic approach used for three-nucleon systems. The reaction amplitude is expanded in terms of a few relevant diagrams computed in momentum space. Energy and momentum are conserved at each vertex. The kinematics and phase space are calculated relativistically, while the nonrelativistic reduction to order $1 / m^{3}$ is done for the elementary operators. ${ }^{4} \mathrm{He}$ and ${ }^{3} \mathrm{H}$ wave functions used in the calculation are solutions of the Urbana group using the Argonne V14 potential. The diagrammatic method relies on a truncated multiple scattering series; it does not treat the final state with the same accuracy as the ground state. The calculation shows that the electrodisintegration of ${ }^{4} \mathrm{He}$ at $P_{\text {rec }} \leq 200 \mathrm{MeV}$ is dominated by one-body mechanisms. Nucleon rescattering is not negligible, but two-body and three-body meson exchange is less important.

\subsection{Medium Dependence of Nucleon Structure}

\subsubsection{Introduction}

Several experimental results throughout the last decades have indicated that there might be medium modifications of the nucleon. Experiments by the European Muon Collaboration (EMC) [25] studying deep inelastic scattering of muons showed that quark momentum distributions in bound nucleons differ from those in a free nucleon. Various theoretical models give different explanations for the effect, but none has so far been able to provide a complete explanation.

While MEC effects change the momentum distribution in the scattering process, they are not very sensitive to form factor changes. Scaling of the variable $y$ [26] should be very sensitive to changes in the form factors. Analyzing previous data, upper limits for form factor changes from $y$-scaling data [5] can be evaluated. For $Q^{2}<0.3 \mathrm{GeV}^{2}$, the change in $G_{M}$ is less than $3 \%$, and less than $10 \%$ for $G_{E}$. An analyis of measurements of the Coulomb sum rule by Jourdan [27] gave an upper limit for a change in $G_{E}$ of $4 \%$ at the one standard deviation level. These findings have sparked new ideas on theoretical 
models of the nucleon. In the following paragraphs, an overview of the most prominent models will be given.

\subsubsection{Theoretical Models}

The nucleon as a composite system is an object of QCD. However, calculating such a complex system is still not possible because of the complicated diagrams which arise from the strong interaction, so the properties of the nucleon, especially when modified by surrounding nuclear matter, can only be described within a model. In the following paragraphs four out of many available models will be described: the swollen nucleon, the soliton, color neutrality and the quark-meson coupling (QMC) model.

\section{Swollen Nucleon}

The swollen nucleon model [28] postulates a change of scale for nucleons in nuclei as result of the medium effects on the quark wave functions. It calculates the form factors on the basis of a Fermi-gas model, assuming that the form factors differ, but the relation between neutrons and protons remain the same. The free nucleon form factors are taken from the dipole fit:

$$
G_{E}=G_{M} / \mu=\left(1+\frac{Q^{2}}{0.71 \mathrm{GeV}^{2}}\right)^{-2}
$$

The modified form factors are determined by comparing results from ${ }^{12} C\left(e, e^{\prime}\right)$ data [29], for which a separation of the longitudinal and transverse structure functions $R_{L}$ and $R_{T}$ has been done at fixed $Q^{2}$, with the results of the Fermi-gas calculation. Incoherent contributions from additional degrees of freedom such as nucleon clusters $(\alpha, \mathrm{d})$ and six-quark clusters are included. The result suggests a $15 \%$ enhancement of the nucleon charge radius, while the mean square radius of the magnetic form factor is almost unchanged. From this increase in the nucleon radius comes a $15 \%$ increase of the magnetic moment, since for massless quarks the magnetic moment is proportional to the size of the quark wave function. In the swollen nucleon model, the fits lead to:

$$
\begin{aligned}
\frac{G_{E}\left(Q^{2}\right)}{G_{E}(0)} & =\left(1+\frac{Q^{2}}{0.54 \mathrm{GeV}^{2}}\right)^{-2} \\
\frac{G_{M}\left(Q^{2}\right)}{G_{E}(0)} & =\left(1+\frac{Q^{2}}{0.69 \mathrm{GeV}^{2}}\right)^{-2}
\end{aligned}
$$




\section{Soliton}

The soliton model is based on the idea that quark confinement should be phenomenologically understandable through a quasi-classical microscopic theory. Confinement could then be described as a phase transition between the interior and exterior of the hadron, thus being a many-body effect. In principle, the QCD vacuum is being treated as a color dia-electric medium very similar to a classical dia-electric medium. While a superconducting state excludes magnetic fields, the QCD vacuum confines color charges by excluding the color electric field. A simple Lagrangian density for such a theory, e.g. in the Friedberg-Lee soliton model [30], is

$$
\mathcal{L}_{\mathrm{FL}}=\bar{\Psi}\left(i \gamma_{\mu} D^{\mu}-m_{N}-f \sigma\right) \Psi-\frac{1}{4} \kappa G_{a}^{\mu \nu} G_{\mu \nu}^{a}+\frac{1}{2}\left(\partial_{\mu} \sigma\right)^{2}-\mathcal{U}(\sigma),
$$

where $\sigma$ is a scalar field, and $\frac{1}{4} \kappa G_{a}^{\mu \nu} G_{\mu \nu}^{a}$ is a dielectric function which is treated perturbatively for colorless states.

There are a variety of nucleon models based on the soliton model. Because the main interest of this work is on the nucleon in nuclear matter, where it clearly must be able to move around, static models will be omitted. An example of a dynamic model is given in [31]. This relativistic model is based on a Lagrangian $\mathcal{L}\left(\Psi_{N}, \sigma, \pi, \omega, \rho\right)$ where nucleons and mesons are degrees of freedom:

$$
\begin{aligned}
\mathcal{L}(x)= & \bar{\Psi}_{N}(x)\left[i \gamma^{\mu} \partial_{\mu}-m_{N}-G_{\sigma N N} \sigma(x)-G_{\omega N N}^{V} \gamma^{\mu} \omega_{\mu}(x)\right] \Psi_{N}(x) \\
& +\frac{1}{2}\left[\partial^{\mu} \sigma(x) \partial_{\mu} \sigma(x)-M_{\sigma}^{2} \sigma^{2}(x)\right]-\frac{1}{4} F_{\mu \nu}^{\omega}(x) F_{\omega}^{\mu \nu}(x) \\
& +\frac{1}{2} m_{\omega}^{2} \omega_{\mu}(x) \omega^{\mu}(x) .
\end{aligned}
$$

The solitons interact by exchanging mesonic fields $\sigma(x)$ and $\omega_{\mu}(x)$. The tensors $G_{\sigma N N}$ and $G_{\omega N N}^{V}$ describe the nucleon-nucleon force of scalar $\sigma$ and vector $\omega$ character, respectively. The tensor $F_{\omega}^{\mu \nu}(x)$ describes the coupling of the $\omega$ fields to the quarks. An additional scalar field $\chi$ serves to bind the system into a finite region, the nucleon. As in the bag models, the nucleon appears as a bubble in the vacuum, but in a fully covariant description. The result is an increase of the soliton radius in nuclear matter as well as changes in the form factors. The modifications are linearly dependent on the density of the nuclear medium. 


\section{Color Neutrality}

This model tries to investigate the role of color neutrality by describing the nucleons' interaction with the nuclear medium in terms of interquark separation within the nucleon. A classical analog to color neutrality is the suppression of interactions when an $e^{+} e^{-}$pair is moving through a charged medium. Similarly, the interaction with the medium is assumed to depend on the relative distances between the quarks. The interaction is also dependent on the density $\rho R$. The calculation of [32] is based on perturbative QCD (pQCD) and treats the nucleon as a superposition of ground state and radial excitations in the framework of relativistic light-front nucleonic model wave functions [33]. The nucleons move in a central potential $V\left(\vec{r}_{i}, \vec{R}\right)$, where $\vec{R}$ is the distance of the nucleon to the center of the nucleus and $\vec{r}_{i}$ is the position of the parton inside the nucleon. Color neutrality requires $V=0$ for $\sum_{i} \vec{r}_{i}=\vec{R}$. Interactions also vanish with $r^{2}=\sum_{i<j}\left(\vec{r}_{i}-\vec{r}_{j}\right)^{2}$. Therefore, the Hamiltonian in first order perturbation theory can be written as

$$
H=V_{0} \rho(R)+V_{0} \rho(R) \frac{r^{2}-\left\langle r^{2}\right\rangle}{\left\langle r^{2}\right\rangle} .
$$

Equation 2.50 includes the effects of color neutrality on the total nuclear mean field and makes no distinction between scalar and vector mean fields. This effect can be studied within the framework of a QMC model, e.g. as developed by [34]. The numerical results for the calculation of $G_{E}$ and $G_{M}$ for the free nucleon and a medium modified nucleon are shown in Fig. 2.7. At higher momentum transfers both medium modified form factors are about $10 \%$ smaller than the free form factors. This is a significant effect, but not in disagreement with Jourdan's analysis [27].

\section{QMC}

In conventional nuclear physics, the electromagnetic interaction of a nucleus is often treated as a combination of different nucleon contributions and various MEC corrections. The Quark Meson Coupling (QMC) model [34] instead examines in a particular quark model for nuclear structure a correction associated with the self consistent 

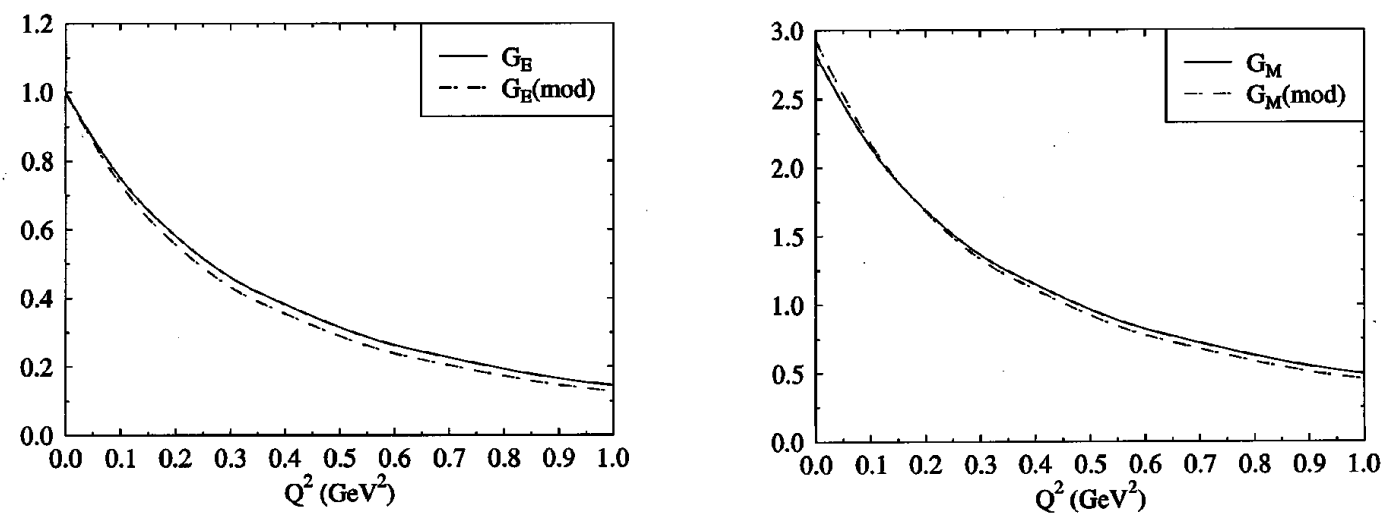

Figure 2.7: The free (solid line) and medium modified (dashed line) form factors $G_{E}$ and $G_{M}$ versus $Q^{2}$ in the color neutrality model. Figure taken from [32].

change in the internal structure of a bound nucleon. It describes the properties of nuclear systems using effective scalar $(\sigma)$ and vector $(\omega)$ fields. Because of the nucleon's substructure the $\sigma$ and $\omega$ fields couple directly to the quarks. No other MEC effects are considered. The nucleon is modeled by the Cloudy Bag Model (CBM), which is an extension of the MIT bag model preserving chiral symmetry [35]. As long as the bag radius $r_{\text {bag }}<0.7 \mathrm{fm}$, the pion field is weak and can be treated perturbatively. Within the QMC model, the saturation energy, density, and compressibility of the nuclear matter are well reproduced. Obvious in-medium changes include the effective masses of the $\rho$, $\omega$ and $\sigma$ mesons. In the mean field approximation, the effective Langrangian density for $\mathrm{QMC}$ is

$$
\begin{aligned}
\mathcal{L}_{\mathrm{QMC}}= & \bar{\Psi}(\vec{r})\left[i \gamma \cdot \partial-m_{N}+g_{\sigma}(\sigma(\vec{r})) \sigma(\vec{r})-g_{\omega} \omega(\vec{r}) \gamma_{0}\right. \\
& \left.-g_{\rho} \frac{\tau_{3}^{N}}{2} b(\vec{r}) \gamma_{0}-\frac{e}{2}\left(1+\tau_{3}^{N}\right) A(\vec{r}) \gamma_{0}\right] \Psi(\vec{r}) \\
& -\frac{1}{2}\left[\left(\nabla \sigma(\vec{r})^{2}\right)+m_{\sigma}^{2} \sigma(\vec{r})^{2}\right]-\frac{1}{2}\left[\left(\nabla \omega(\vec{r})^{2}\right)+m_{\omega}^{2} \omega(\vec{r})^{2}\right] \\
& +\frac{1}{2}\left[(\nabla b(\vec{r}))^{2}+m_{\rho}^{2} b(\vec{r})^{2}\right]+\frac{1}{2}(\nabla A(\vec{r}))^{2}
\end{aligned}
$$

where $\Psi(\vec{r}), \sigma(\vec{r}), \omega(\vec{r}), b(\vec{r})$ and $A(\vec{r})$ are the nucleon, $\sigma, \omega, \rho$ and Coulomb fields, respectively. The electric and magnetic form factors for a bound proton calculated in the local-density approximation are given by:

$$
G_{E, M}^{\alpha}\left(Q^{2}\right)=\int G_{E, M}\left(Q^{2}, \rho(\vec{r})\right) \rho_{p \alpha}(\vec{r}) d \vec{r}
$$




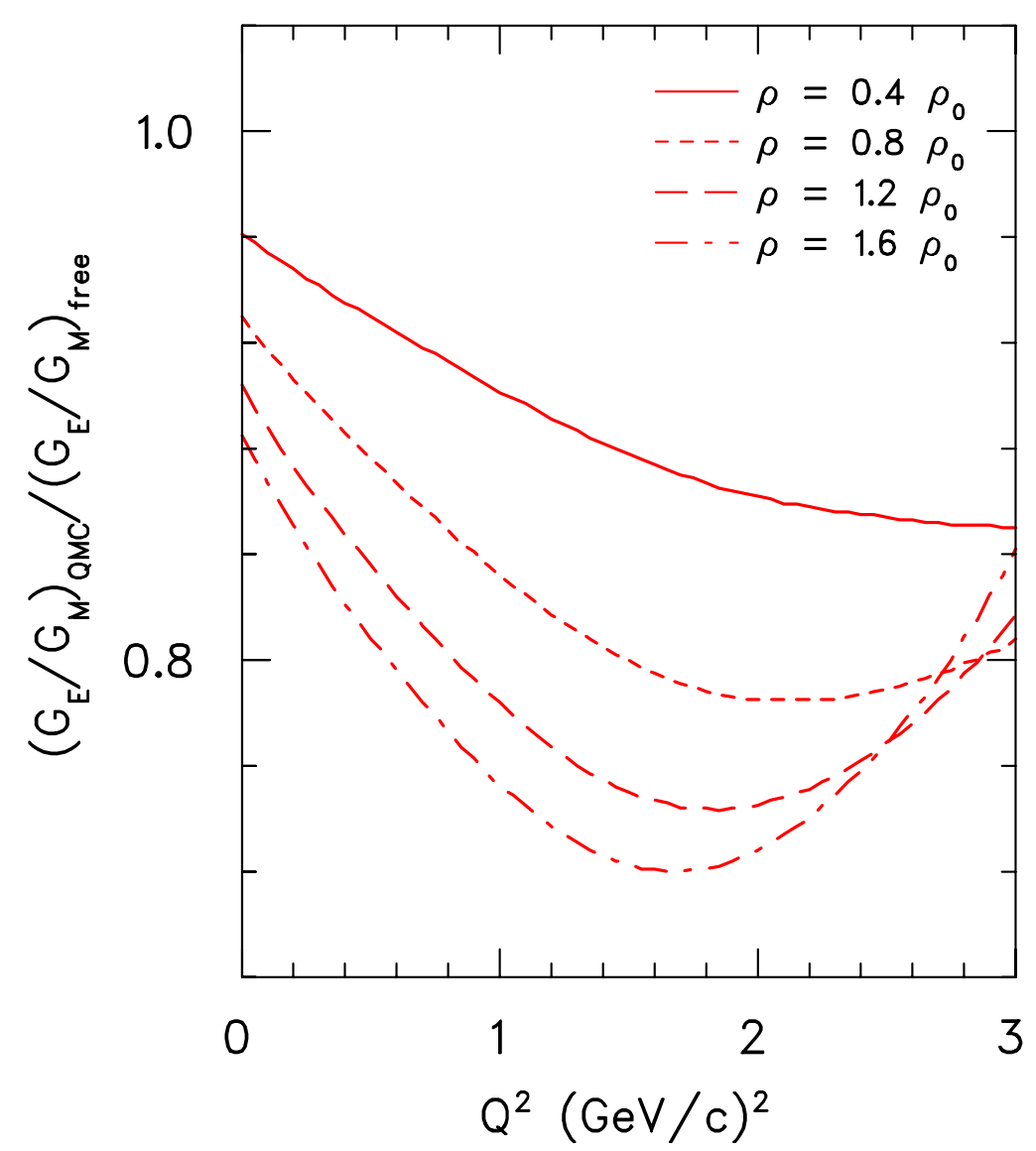

Figure 2.8: Medium effects on the form factor ratio $G_{E} / G_{M}$ vs. $Q^{2}$ as calculated in the QMCD model. The form factor change is plotted for different nuclear densities with $\rho_{0}$ being the density of bulk nuclear matter.

where $\alpha$ denotes an orbit with appropriate quantum numbers, and $\rho_{p \alpha}(\vec{r})$ is the local baryon density for the immersed proton with the form factor $G_{E, M}\left(Q^{2}, \rho(\vec{r})\right)$. QMC calculations reveal that the charge form factors are much more sensitive to the medium than the magnetic form factors. This results in a change of the form factor ratio $G_{E} / G_{M}$ as shown in Fig. 2.8. An open question is whether the bag constant decreases with increasing density. 


\section{Chapter 3}

\section{Experimental Setups at MAMI and TJNAF}

The experiment was performed at two laboratories, at the Mainz Microtron (MAMI) of Johannes Gutenberg University in Mainz, Germany, and at the Thomas Jefferson National Accelerator Facility (JLab) in Newport News, Virginia. After an overview of the kinematics, the experimental setup for MAMI and JLab concerning the respective accelerator, the setup in the spectrometer halls and the data acquisition system will be described.

\subsection{Kinematics}

The previous section showed how measuring polarization transfer in quasielastic scattering can be used to learn about the properties of the nucleon in the medium. A coincidence measurement ${ }^{4} \mathrm{He}\left(\vec{e}, e^{\prime} \vec{p}\right)^{3} \mathrm{H}$ was made in the quasielastic scattering region in near parallel kinematics. For the incident electron beam the beam energy, polarization, and position of the rastered beam on the target cell was determined. To establish the four-momentum transfer $q_{\mu}$ and the electron scattering plane, the momentum and angle of the scattered electron was measured with a magnetic spectrometer. A second magnetic spectrometer was used for momentum and angle determination of the knockout proton. For measuring the induced and transferred polarization of the knockout proton, the hadron arm was equipped with a focal plane polarimeter (FPP). The information of both spectrometers together allows the calculation of the reaction vertex and the determination of the missing mass, which allows the isolation of the ${ }^{3} \mathrm{H}$ final state. To measure the polarization ratio for the free proton and to study systematic effects, ${ }^{1} \mathrm{H}\left(\vec{e}, e^{\prime} \vec{p}\right)$ elastic scattering data were taken at the same nominal settings as for the ${ }^{4} \mathrm{He}$ measurements. 
The experiments at five different momentum transfers $Q^{2}$ from $0.4 \mathrm{GeV}^{2}$ to $2.6 \mathrm{GeV}^{2}$, were done at MAMI for the lowest $Q^{2}$, and at JLab for the higher $Q^{2}$. Table 3.1 lists the reaction kinematics, spectrometer setup and experimental facility used for data taking. Section 3.2 describes the experimental setup used in Mainz, section 3.3 describes the setup at JLab.

\begin{tabular}{ccccccccc}
\hline \hline $\begin{array}{c}Q^{2} \\
\left(\mathrm{GeV}^{2}\right)\end{array}$ & Target & $\begin{array}{c}E_{0} \\
(\mathrm{GeV})\end{array}$ & $\begin{array}{c}\theta_{p} \\
(\mathrm{deg})\end{array}$ & $\begin{array}{c}p_{p} \\
(\mathrm{GeV})\end{array}$ & $\begin{array}{c}\theta_{e} \\
(\mathrm{deg})\end{array}$ & $\begin{array}{c}p_{e} \\
(\mathrm{GeV})\end{array}$ & $\begin{array}{c}d \\
(\mathrm{~cm})\end{array}$ & Lab \\
\hline 0.4 & ${ }^{1} \mathrm{H}$ & 0.855 & 48.16 & 0.660 & 50.24 & 0.625 & 7.0 & Mainz \\
0.4 & ${ }^{4} \mathrm{He}$ & 0.855 & 46.56 & 0.660 & 50.24 & 0.625 & 7.0 & Mainz \\
0.5 & ${ }^{1} \mathrm{H}$ & 3.402 & 63.12 & 0.765 & 12.45 & 3.103 & 11.4 & JLab \\
0.5 & ${ }^{4} \mathrm{He}$ & 3.402 & 61.42 & 0.765 & 12.45 & 3.103 & 11.4 & JLab \\
1.0 & ${ }^{1} \mathrm{H}$ & 4.238 & 54.82 & 1.148 & 14.58 & 3.667 & 34.3 & JLab \\
1.0 & ${ }^{4} \mathrm{He}$ & 4.238 & 53.50 & 1.148 & 14.58 & 3.667 & 34.3 & JLab \\
1.5 & ${ }^{1} \mathrm{H}$ & 4.238 & 46.76 & 1.547 & 19.33 & 3.341 & 45.7 & JLab \\
1.5 & ${ }^{4} \mathrm{He}$ & 4.238 & 46.76 & 1.547 & 19.33 & 3.341 & 45.7 & JLab \\
2.6 & ${ }^{4} \mathrm{He}$ & 4.238 & 36.20 & 2.159 & 27.08 & 2.797 & 49.5 & JLab \\
\hline \hline
\end{tabular}

Table 3.1: Table of kinematics. $E_{0}$ is the beam energy, $\theta_{p}\left(\theta_{e}\right)$ the proton (electron) spectrometer angle, $p_{p}\left(p_{e}\right)$ the proton (electron) spectrometer central momentum and $d$ is the carbon analyzer thickness.

\subsection{Mainz Experiment A1/2-93}

The first part of the experiment took place in Hall A1 of MAMI. The experiment ran in June/July 1999 for 17 days of data taking. The following sections describe the MAMI accelerator and the instrumental equipment of Hall A1.

\subsubsection{MAMI}

MAMI is an electron accelerator consisting of three cascaded racetrack microtrons [36]. Each racetrack microtron consists of a linac which accelerates the electrons and two large dipoles (see Fig. 3.1). After being accelerated though the linac, the beam makes a $180^{\circ}$ turn in one dipole, travels through a vacuum beamline (not shown in the drawing) and 


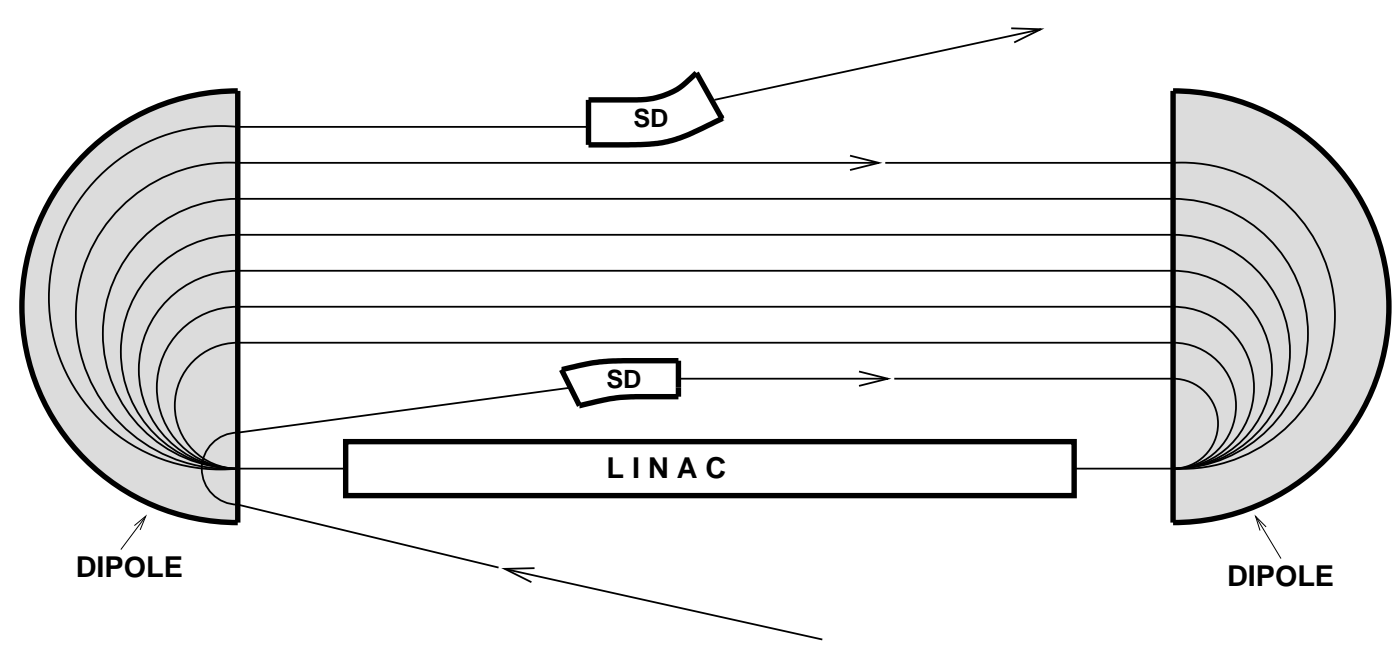

Figure 3.1: Scheme of a racetrack microtron. A linac accelerates the electrons. Two large dipole magnets (shaded areas) provide the recirculation. Smaller steering dipole magnets (SD) are used for steering the incoming and exiting beam.

gets recirculated into the linac by the second dipole, which closes the racetrack-shaped electron path through the microtron. The linacs consist of 1-5 Klystrons driven by an RF frequency of $2.45 \mathrm{GHz}$. Smaller steering magnets (D) control the entrance and exit of the beam. The maximum achievable energy gain in a microtron is about a factor of ten. With an injector energy of 2-3 MeV it requires three cascaded microtrons to reach the final energy of $855 \mathrm{MeV}$. MAMI provides a duty factor of $100 \%$ and extremely high beam quality concerning the beam halo, emittance and stability [37]. The facility has two electron sources for unpolarized and polarized electrons. For this experiment, only the polarized electron source was used; a detailed description of the source can be found in [38]. Polarized electrons are produced by photo-emission from III/V semiconductors, in this case a strained GaAsP photo-cathode with a submonolayer surface of cesium. The photoemission is excited by a circularly polarized laser beam; this provides high brilliance and fast helicity change via switching of the laser light polarization. The laser pulse frequency of $2.45 \mathrm{GHz}$ with a bunch length of 50 ps matches the linac frequency and results in a transmission from the source to the target of $>90 \%$ [39]. To compensate for the loss of quantum efficiency, the laser beam spot on the crystal was moved every few hours and the crystal surface was recesiated approximately every 48 hours. With this polarized source the maximum beam current achieved is about $15 \mu \mathrm{A}$; maximum beam polarizations are about $80 \%$. The beam polarization was measured with a Mott 


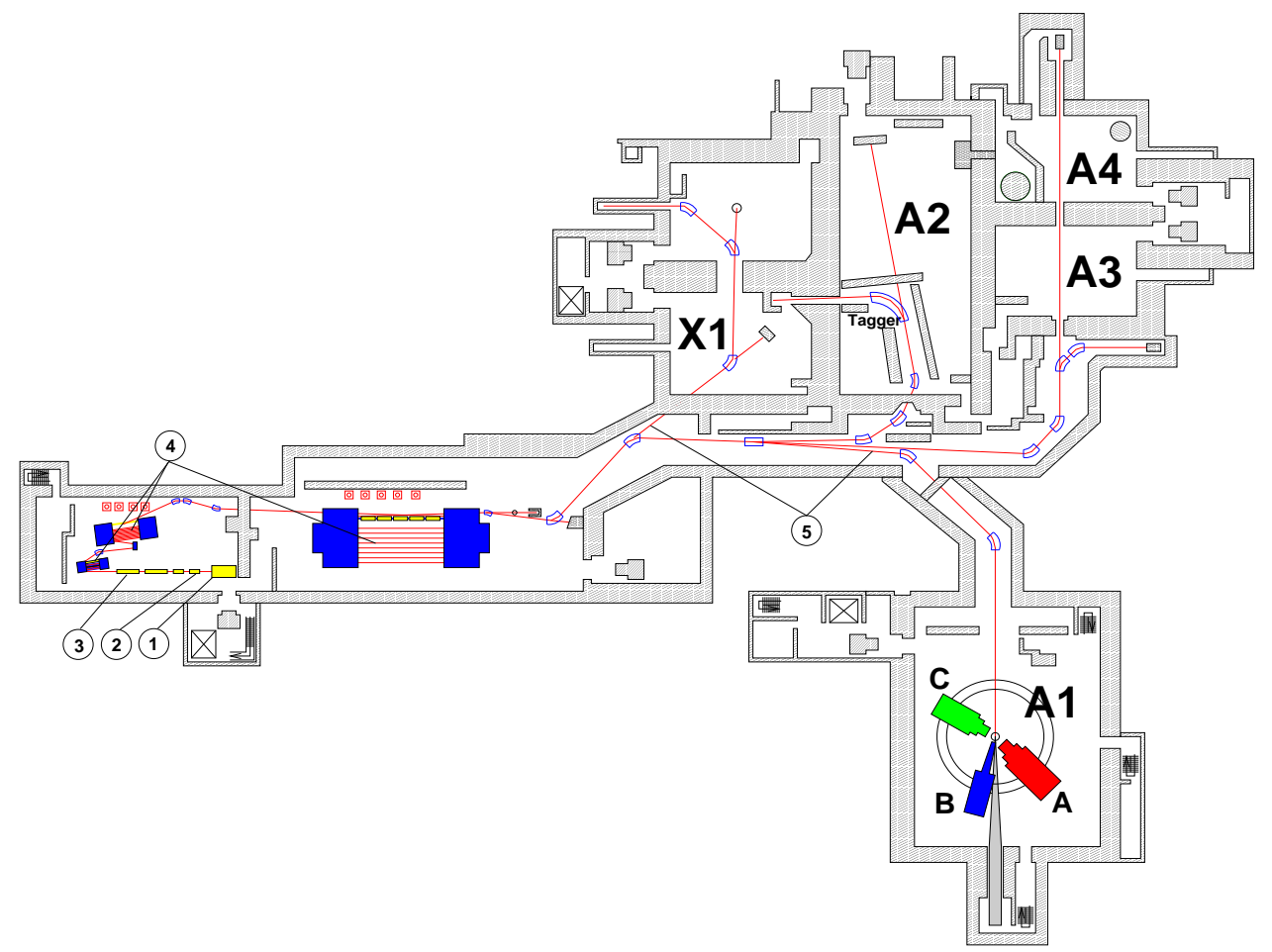

Figure 3.2: Floorplan of MAMI. (1) is the unpolarized electron gun, (2) the polarized gun. (3) is the preaccelerating linac. (4) are the three microtron stages. Several beamlines (5) transport the beam into the experimental halls (X1, A1, A2, A3, A4).

polarimeter $[38,40]$. For Mott polarimetry, the $100 \mathrm{keV}$ electrons extracted from the source are scattered off a gold foil. The polarization dependent part of the cross section produces a left-right asymmetry, which is measured by two detectors placed at the same angle left and right of the beam. To reduce systematic uncertainties, the beam polarization is switched. The beam position is measured at various positions in and in between the three microtrons by wire scanners, synchrotron radiation monitors and fluorescent screens [41]. Additionally, the beam position and current is measured in the last RTM by RF position monitors in the $\mathrm{TM}_{110}$ mode $[42,43]$.

After extraction from the source, preacceleration in a linac, and acceleration in the three microtron stages electrons are delivered via the beamline into one of the different experimental halls. Figure 3.2 shows the floor plan of MAMI and the connected experimental halls. Hall A1 provides a unique facility for electron scattering experiments. As shown in Fig. 3.3 it is equipped with three magnetic spectrometers $\mathrm{A}, \mathrm{B}$, and $\mathrm{C}$, which can be used simultaneously. An extensive description of A1 including properties of the 


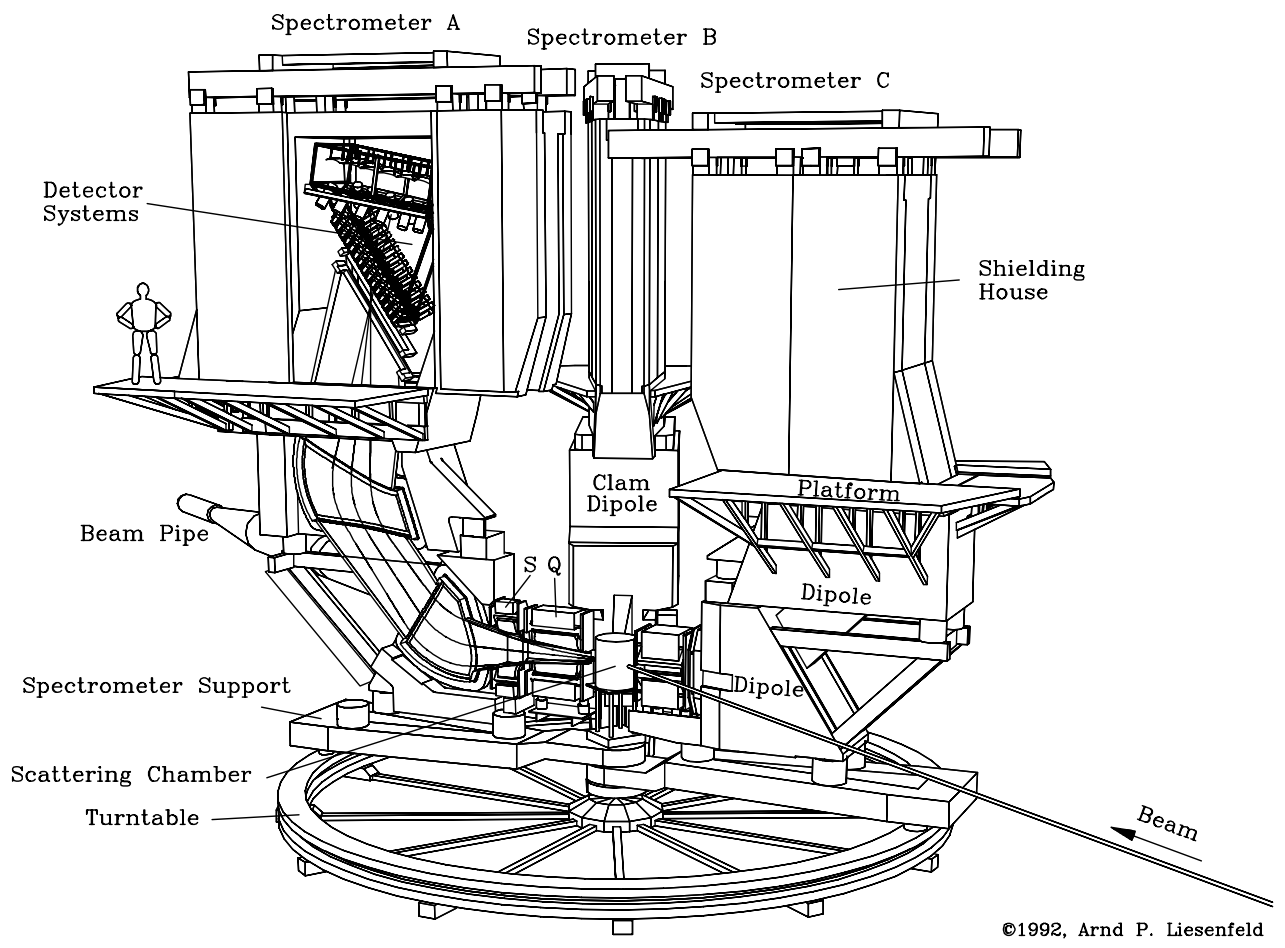

Figure 3.3: Hall A1 at MAMI. The beam comes from the lower right and hits the target in the scattering chamber. Scattered charged particles are detected in the three magnet spectrometers A, B and C. They are mounted on a turntable which allows to rotate the spectrometers around a common axis. Unscattered beam electrons are collected in a Faraday cup behind the outgoing beam pipe. Figure provided by A. Liesenfeld.

spectrometers and detectors can be found in [44] and [45].

\subsubsection{Experimental Setup}

As target setup we used the high pressure ${ }^{4} \mathrm{He}$ cryogenic target [46]; the gas had a temperature of $19 \mathrm{~K}$ and a pressure of 19 bar. For the hydrogen measurements the same cell, filled with liquid hydrogen, was used. Thin carbon and BeO targets were used for calibrations and beam diagnostics. Reference [47] contains a detailed description of the cryogenic system. To avoid damage to the thin target cell walls by the high energy density beam, the beam was rastered. Spectrometer A, equipped with an FPP [48], was used to measure the knockout protons. The electrons were detected with spectrometer B. Essential properties of the spectrometers are listed in Table 3.2. 


\begin{tabular}{lcc}
\hline \hline & $\mathrm{A}$ & $\mathrm{B}$ \\
\hline Configuration & QSDD & $\mathrm{D}$ \\
Dispersive plane & point-point & point-point \\
Nondispersive plane & parallel-point & point-point \\
Maximum momentum $(\mathrm{MeV} / \mathrm{c})$ & 735 & 870 \\
Reference momentum $(\mathrm{MeV} / \mathrm{c})$ & 630 & 810 \\
Central momentum $(\mathrm{MeV} / \mathrm{c})$ & 665 & 810 \\
Solid angle (msr) & 28 & 5.6 \\
Momentum acceptance & $20 \%$ & $15 \%$ \\
Dispersive angular acceptance (mrad) & 70 & 70 \\
Nondispersive angular acceptance $(\mathrm{mrad})$ & 100 & 20 \\
Long target acceptance $(\mathrm{mm})$ & 50 & 50 \\
Angle of focal plane & $45^{\circ}$ & $45^{\circ}$ \\
Length of focal plane $(\mathrm{m})$ & 1.80 & 1.80 \\
Length of central trajectory $(\mathrm{m})$ & 10.75 & 12.03 \\
Central dispersion $(\mathrm{cm} / \%)$ & 5.77 & 8.22 \\
Central magnification & 0.53 & 0.85 \\
Momentum resolution & $10^{-4}$ & $10^{-4}$ \\
Angular resolution at target (mrad) & $\leq 3$ & $\leq 3$ \\
Position resolution at target (mm) & $3-5$ & 1 \\
\hline \hline
\end{tabular}

Table 3.2: Table of optical properties for spectrometer A and B, numbers taken from [53]

\section{Target}

The cryotarget system in Hall A1 was constructed in Saclay [49]. In recent years, it was extended $[46,50]$ and now consists of the cryo-system with nitrogen reservoir, which is connected via a $12 \mathrm{~m}$ transfer line to the main target mounted in the scattering chamber. The cooling power of the cryo-system is about $100 \mathrm{~W}$, with a loss of about $44 \mathrm{~W}$ in the transfer line. Figure 3.4 shows the cryo-system in its current status. A schematic view of the target loop is shown in Fig. 3.5. The system is a closed loop. The cryogenic gas or liquid enters from the top and is pumped by a fan. Energy deposited in the target by the beam or other sources is removed in the heat exchanger. The target cell [51] is made of a very rigid aluminum alloy. It was milled out of a solid aluminum 


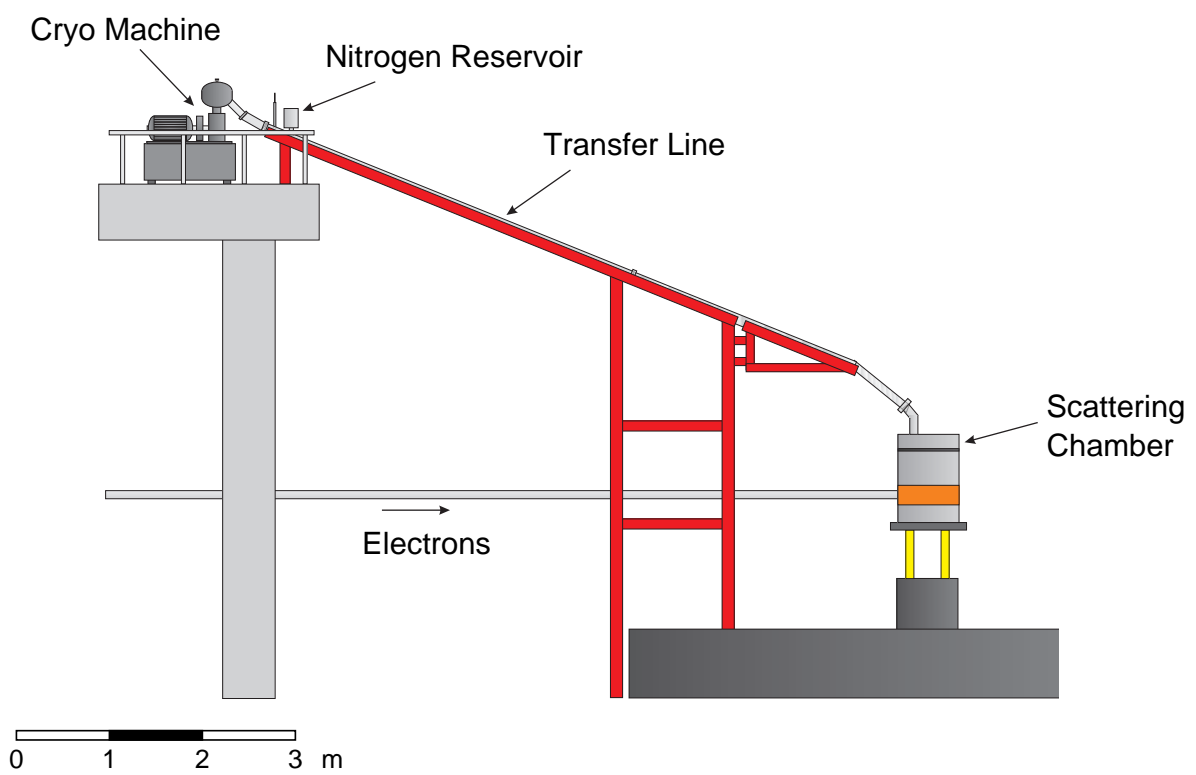

Figure 3.4: Cryo-system of Hall A1. The liquifier is placed on a gallery and connected to the target chamber by a nitrogen shielded transfer line. The cryotarget is mounted in the scattering chamber. Figure taken from [46]. 


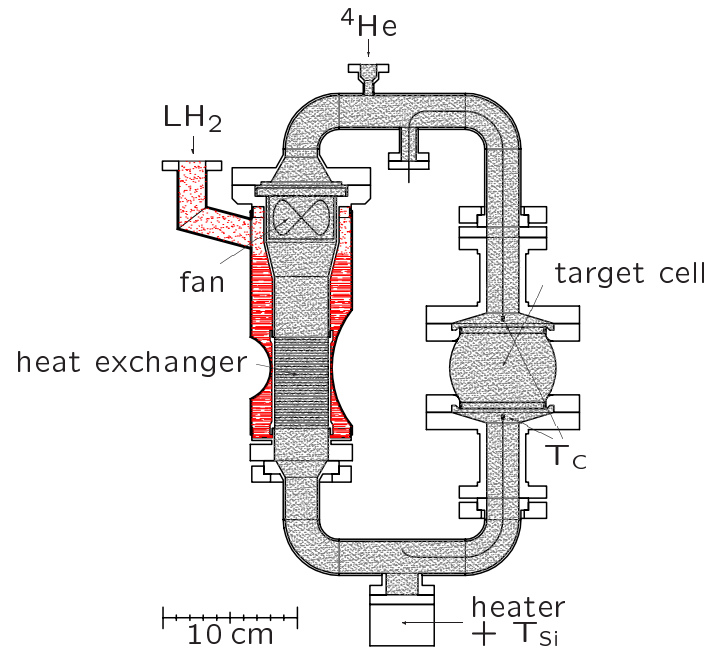

Figure 3.5: Schematic view of the target loop. Figure taken from [46].

block, which gives it a high mechanical stability. The beam entry window is a $2 \mathrm{~cm}$ high strip with an average thickness of $250 \mu \mathrm{m}$. The cell is commissioned for pressures up to $200 \mathrm{kPa}$ with a working temperature of $20 \mathrm{~K}$. In this experiment, the cell was filled with cryogenic ${ }^{4} \mathrm{He}$ gas at a temperature of $29 \mathrm{~K}$ and pressure of $190 \mathrm{kPa}$. For the hydrogen measurement, the cell was for the first time filled with liquid hydrogen.

\section{Spectrometer A}

Spectrometer A was used as proton spectrometer in the experiment. Its magnetic elements consist of one quadrupole (Q), a sextupole (S), and two dipoles (D). The magnet configuration QSDD was chosen to achieve several optical properties: a large acceptance, good "hardware" momentum resolution and a flat focal plane. The quadrupole focuses in the nondispersive $y$-direction to enlarge the scattering angle acceptance. The sextupole magnet serves to compensate for spherical aberrations of the quadrupole and 


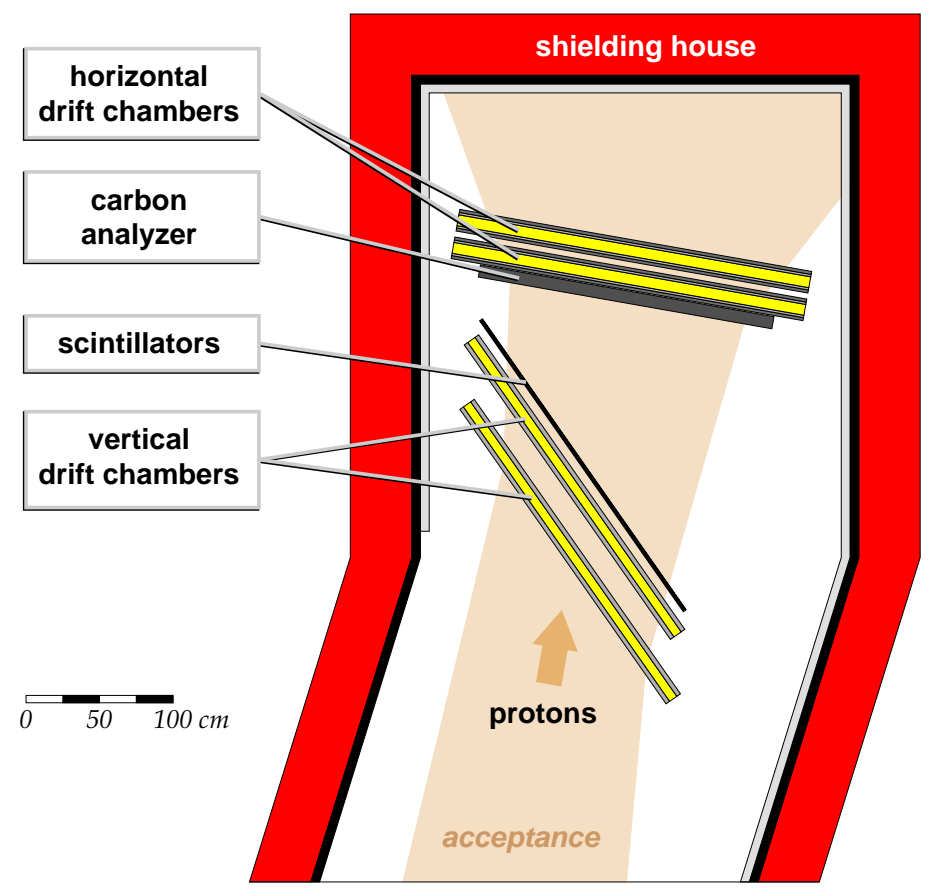

Figure 3.6: Detector house of spectrometer A. The detectors are protected from background radiation by a shielding hut. After traveling through the QSDD magnet system (not shown in the picture), protons cross the VDCs and both scintillator planes before crossing the carbon analyzer and the HDCs. The shaded area indicates the acceptance. Figure taken from [48].

the dipole magnets. The dipoles create the dispersion needed for momentum separation. Special shaping of the dipole entrance and exit provides additional quadrupole and sextupole strength. In addition the magnetic field also rotates the spin of the proton while it moves through the spectrometer.

In Fig. 3.6 a schematic view of the spectrometer A detectors including the FPP is shown. The detector package of spectrometer A consists of two double-planes of vertical drift chambers (VDC) which provide particle tracking in position and angle, and two planes of scintillation detectors for timing, particle identification and trigger.

A VDC consists of a plane of signal and potential wires at ground potential enclosed by two cathode foils at $-6400 \mathrm{~V}$. The signal wires with diameters of $15 \mu \mathrm{m}$ are $5 \mathrm{~mm}$ apart and interspersed with the thicker potential wires. A drift cell is defined so that its center is a signal wire and its width and height $5 \mathrm{~mm}$ and $24 \mathrm{~mm}$, respectively. The electrical field resulting from this setup consists of parallel field lines which are 


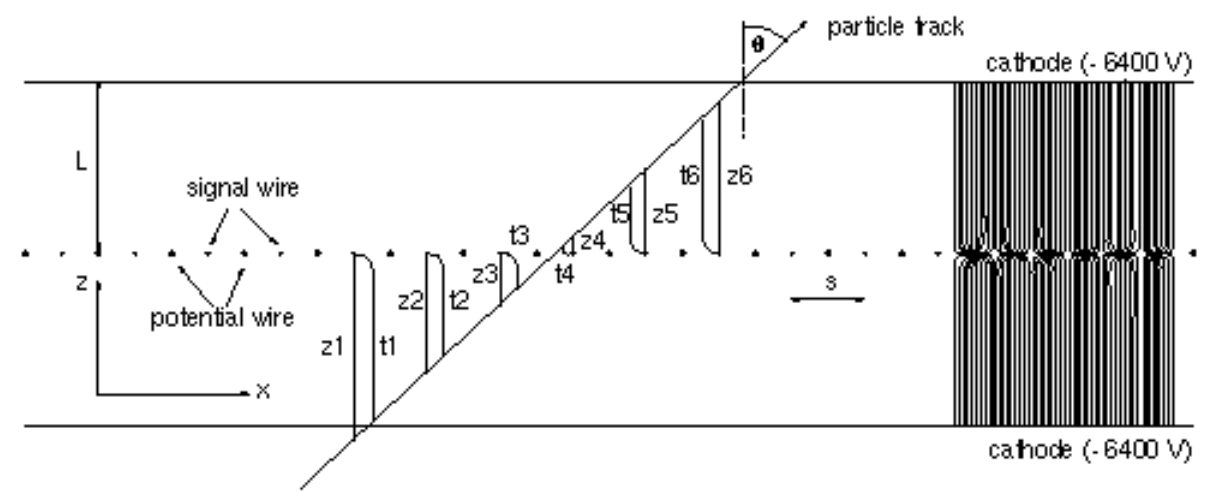

Figure 3.7: Working principle of a VDC. The field and potential wires are placed at a distance L parallel to the cathodes; the resulting field lines are shown on the right side of the figure. The distance between two signal (potential) wires is s. A particle crossing the chamber at an angle $\theta$ ionizes the chamber gas. The electrons produced at a distance $z_{i}$ from the $i^{t h}$ wire drift for the time $t_{i}$.

perpendicular to the cathode foils, with deviations only close to the wires as shown in Fig. 3.7. The VDC is filled with equal parts of argon and isobutane and a $1.5 \%$ admixture of pure ethanol.

A proton transversing the VDC ionizes the gas molecules. The resulting electrons drift with an average velocity of $25 \mu \mathrm{m} / \mathrm{ns}$ to the signal wire. Close to the signal wire the high field gradient accelerates electrons to energies high enough to ionize other molecules, hence a charge avalanche is created. The charge signal on the wire gets electronically preamplified before it is digitized for readout. The drift distances of the particle track to the signal wires along the $z$-direction, $z_{1}$ to $z_{5}$, can be calculated by measuring the drift time $t_{i}$ and the known drift velocity (see Fig. 3.7). The VDCs are mounted inside a shielding house at a $45^{\circ}$ angle to the reference particle trajectory. This results in a signal in 5-6 wires, which increases the total efficiency of the chambers. The median of drift cells measuring a signal is five for the $x$-chambers and four for the $s$-chambers. With a least-squares fit through the drift distances the position and angle of the detected particle is calculated. Because one VDC alone can only provide a twodimensional projection of the true particle track, at least two chambers are needed for complete tracking. To improve angular resolution, a second pair of VDCs is used. With this set of four drift chambers a position resolution of $94 \mu \mathrm{m}$ in the dispersive direction results in a momentum resolution of $1 \times 10^{-4}(\mathrm{FWHM})$. 
The plastic scintillators, made of NE 102A, consist of two planes parallel to the VDCs with 15 paddles each. The planes have a lateral offset of half a paddle width. The plane closest to the VDC has a thickness of $3 \mathrm{~mm}$ and serves as a $\Delta \mathrm{E}$ energy loss detector, while the second plane with a thickness of $1 \mathrm{~cm}$ is used for the fast timing signal and time of flight (ToF) measurements. Each scintillator paddle is read out via plastic light guides by photomultiplier tubes from both sides. Due to the different light path length of the signal before it reaches the tube, the timing signal would be about 5 ns wide. This can be improved by more than an order of magnitude by using the information of the particle impact point provided by the VDCs.

\section{Mainz FPP}

The FPP [48] of spectrometer A consists of two parts, the carbon analyzer block and a tracking detector for measuring the proton track after the carbon analyzer. To enable measurements over a large range of proton energies, the carbon analyzer is made of three layers with a thickness of $4 \mathrm{~cm}, 2 \mathrm{~cm}$ and $1 \mathrm{~cm}$, each layer split in two segments. To avoid protons leaking through the slit between the segments of a layer, the edges were cut at an angle of $35^{\circ}$. With an area of $200 \mathrm{~cm} \times 60 \mathrm{~cm}$ the carbon block is large enough to cover the whole spectrometer acceptance.

The rear tracking detector consists of two pairs of horizontal drift chambers (HDC). In each pair, the direction of the signal wires is rotated $45^{\circ}$ to the middle plane of the spectrometers in such a way that the wires of the chambers within a pair are perpendicular to each other. Horizontal drift chambers consist of a plane of parallel wires sandwiched between two grounded cathode foils. The gap between the cathodes is filled with a gas mixture of $80 \%$ ethane and $20 \%$ of an argon-ethanol mixture. The field forming signal and potential wires have a distance of $1 \mathrm{~cm}$ to each other and the cathode foils. The potential wires are on the same potential as the cathodes; the potential difference to the field wires is $+3 \mathrm{kV}$. Therefore the electron drift lines are as indicated in Fig. 3.8. When a proton goes through an HDC chamber, it ionizes the chamber gas. The electrons drift along the field lines to the signal wires, while the cations drift to the potential wire. The field gradient close to the wires causes a 


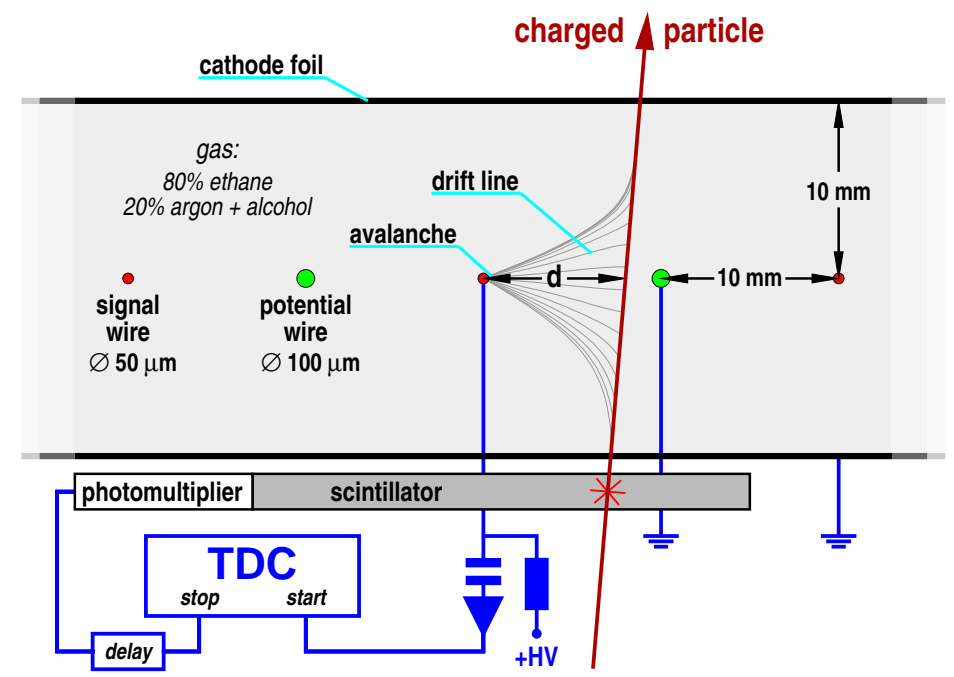

Figure 3.8: Schematics of the HDC. The field forming signal and potential wires have a distance of $1 \mathrm{~cm}$ to each other and to the cathode foils. Electrons from the ionization along the particle track drift to the signal wire. The drift time measurement is started by the individual signal wires and stopped by a fast external trigger scintillator. Fig. taken from [52].

charge avalanche to develop. The readout electronics registers the charge signal and the wire position. A specifically developed electronics registers which side of the signal wire the proton passed through. From the combined information of all four chambers the position can be determined with a resolution of $300 \mu \mathrm{m}$, and the angle with a resolution of $2 \mathrm{mrad}$. This is mainly due to the uncertainty in the drift time to drift distance relation.

\section{Spectrometer B}

Spectrometer B was used as electron spectrometer. It consists of an inhomogeneous dipole magnet and a tracking detector system. Its magnet is constructed as a double focusing "clamshell", which means the dipole is open like a clam with an angle of $3.495^{\circ}$. The non-uniform field leads to point-to-point focusing in the dispersive and in the non-dispersive plane. Spectrometer B can be used for out of plane measurements up to a maximum angle of $10^{\circ}$; our measurement was done in plane. The detectors consist of a set of four VDCs and two planes of scintillators, the setup being very similar to spectrometer A. The optical properties are listed in Table 3.2. 


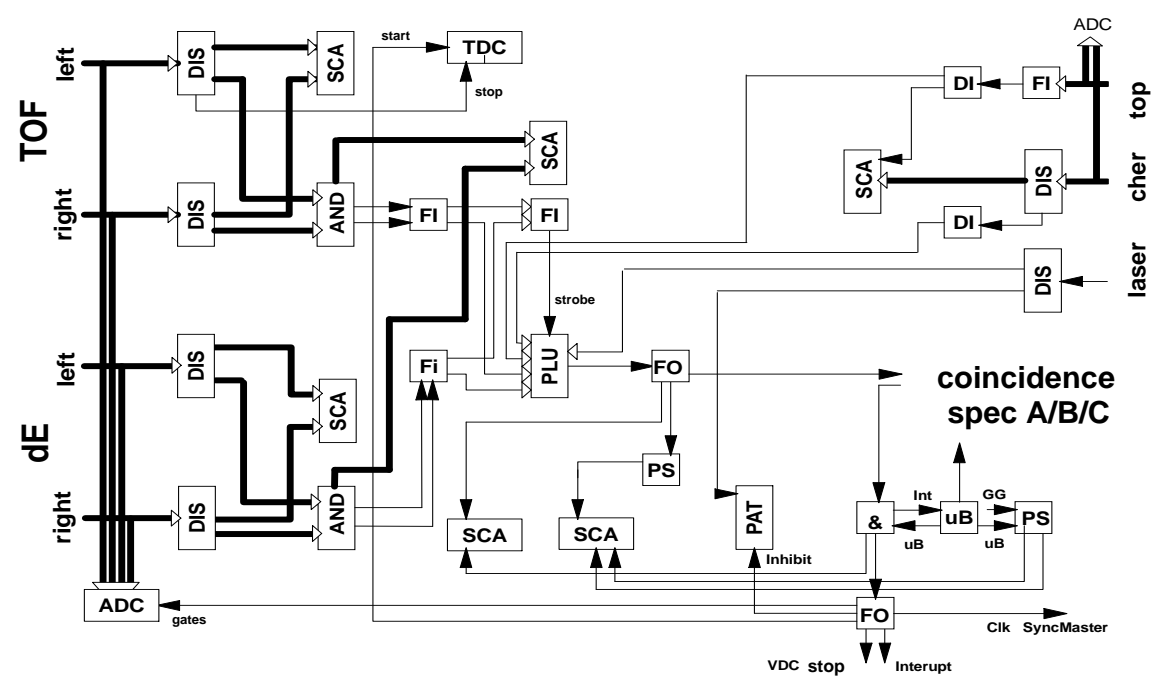

Figure 3.9: Local circuit for the trigger detector system; $\mathrm{dE}$ and ToF denote the signals from the first and second scintillator plane, respectively. Legend: dE, TOF, top, cher: photomultiplier signals from the detectors, ADC/TDC: Analog/Time to Digital Converter, FI/FO: Fan In/Fan Out, SCA: Scaler, DIS, DI: Discriminator, PS: Prescaler, uB: microBusy, PLU: Programmable Logic Unit. Fig. taken from [44].

\subsubsection{Trigger and Data Acquisition}

Generating the trigger signal of spectrometer A and B requires a signal in both scintillator planes. To minimize noise, both photomultipliers on a scintillator paddle must read out a signal in coincidence. The processing of the trigger signal from the scintillators is done by a Programmable Logic Unit (PLU LeCroy 4508). A schematic view of the spectrometer A trigger is shown in Fig. 3.9; the trigger of spectrometer B is similar. The local trigger signal is processed by a second, central coincidence PLU, which generates the interrupt to the local data acquisition if the required coincidence pattern, in this case "A and B", is recognized. It is also possible for calibration purposes to add prescaled single events to the data stream, e.g. only 1 out of 1000 single electron events enters the data stream. In addition, this trigger pattern is also used to mark the event for the local event readout, which is later needed by the event builder. The event readout was done locally on each spectrometer with a VMEbus-System. The system contained a master/slave multiprocessor system using a E6-CPU (Motorola 68030) as master and a E5-CPU (Motorola 68020) as slave. The realtime E5-CPU was used for 
fast data acquisition, while the master processor was packaging the data and sending it to the host via an Ethernet connection. A data acquisition workstation is used to merge the data streams from the different spectrometers. A specially designed program package, the "Eventbuilder" $[54,55]$, uses the above mentioned trigger marks to match the corresponding events to a single scattering event. The events are stored on a hard drive. From there the data is archived on CD-ROM or tape. The software package MECDAS [55-57] ( Mainz Experiment Control and Data Acquisition System) is used for this procedure. The online and offline analysis was done with the software package Cola ++ . At the time of this experiment the maximum data acquisition rate was $200 \mathrm{~Hz}$ with $20 \%$ deadtime, limited by the front-end CPUs.

\subsection{Jefferson Lab E93-049}

The second part of the experiment took place in Hall A at JLab. Experiment 93-049 (Polarization Transfer in the Reaction ${ }^{4} \mathrm{He}\left(\vec{e}, e^{\prime} \vec{p}\right)^{3} \mathrm{H}$ in the Quasielastic Scattering Region, spokespersons R. Ent and P. Ulmer) ran in April/May 2000 for 28 days of data taking and 6 days of detector calibrations. The following sections describe the CEBAF accelerator and the instrumental equipment of Hall A.

\subsubsection{CEBAF}

The Continuous Electron Beam Accelerator Facility (CEBAF) is a superconducting, high current, high-duty-factor accelerator with a maximum beam energy of $5.5 \mathrm{GeV}$. An accelerator site plan is shown in Fig. 3.10.

The polarized source [58] provides up to $78 \%$ polarized electrons with a maximum current of $100 \mu \mathrm{A}$ which are preaccelerated to $45 \mathrm{MeV}$ by the injector. The beam is then further accelerated by the two main linacs, which are connected by $180^{\circ}$ recirculation arcs. Up to five recirculations (5-pass) are possible. Each linac consists of cryo-modules, each containing eight cryo-units consisting of five cell elliptical cavities. The cryo-units are made of niobium and are cooled to $2 \mathrm{~K}$. They are driven by an RF frequency of 1.497 GHz. Three beams can be accelerated simultaneously and are divided in the 


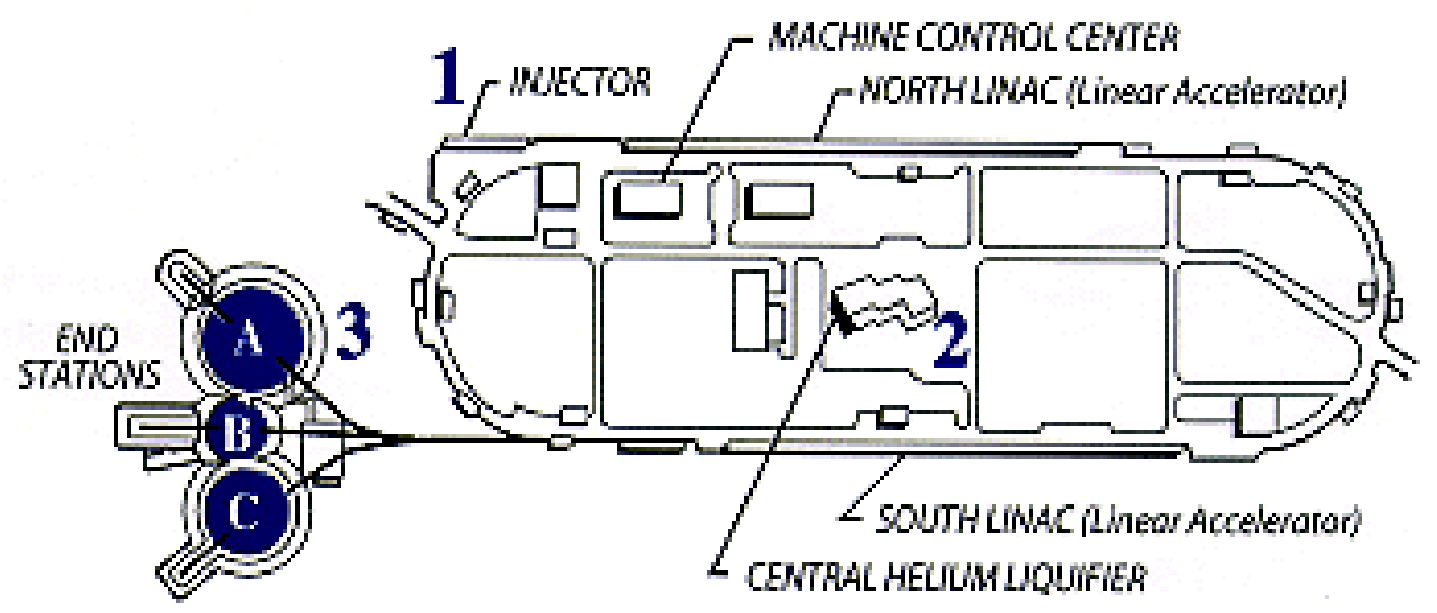

Figure 3.10: Site plan of the CEBAF accelerator. After preacceleration in the injector (1), the beam is accelerated through the north and south linac up to five times before it is distributed to the end stations (3) in the beam switchyard. The helium used for cooling superconducting systems is produced in the central helium liquifier (2).

beam switchyard into the three Halls, A, B, and C, with Halls A and C taking high current beams up to $130 \mu \mathrm{A}$ and Hall B low currents of a few $\mathrm{nA}$.

\subsubsection{Beam and Beam Diagnostics}

\section{Beam Energy Measurement}

At the time of the experiment two independent methods to measure the beam energy in Hall A were available, "ep" and "ARC". In the $e p$ method, the ${ }^{1} \mathrm{H}\left(e, e^{\prime} p\right)$ reaction is used to determine the beam energy. The setup [59] is a stand-alone device located $17 \mathrm{~m}$ upstream of the target. The target consists of a moving $\mathrm{CH}_{2}$ tape with a thickness of 10-30 $\mu \mathrm{m}$. The two proton detectors and both electron silicon microstrip detectors are arranged symmetrically to the beam axis in the reaction plane. Making simultaneous measurements with both arms cancels uncertainties due to beam position and direction to first order. The beam energy is given to first order by

$$
E=m_{p} \frac{\cos \theta_{e}+\sin \theta_{e} / \tan \theta_{p}-1}{1-\cos \theta_{p}}
$$

The ARC energy measurement method uses the connection between the beam energy $E$, the bend angle $\theta$ through the arc section of the beamline, the field integral 
$\int \vec{B} d \vec{l}$ and the constant $c$ :

$$
E=c \cdot \frac{\int \vec{B} d \vec{l}}{\theta} .
$$

The method consists of two simultaneous measurements. The bend angle is measured by two monitors determining the direction of the beam upstream and downstream of the arc, respectively. Each monitor consists of two beam position monitors ("superharps"). The field integral is measured in a dipole magnet identical to the eight dipole magnets located in the arc. This ninth dipole is kept at the same conditions and is connected in series to the arc dipoles. The field integral of the central and fringe field is measured with NMR probes in the ninth dipole.

Repeated measurements of beam energies over a period of several years show good agreement with each other, resulting in an uncertainty of the beam energy $\left(\leq 4 \cdot 10^{-4}\right)$. One exception is a region around $3 \mathrm{GeV}$, where the $e p$ results are lower than the ARC results. It is conjectured that a microstrip used in this energy region could be misaligned. An investigation of the problem is ongoing.

\section{Beam Position Monitors}

To monitor the position of the beam along the beamline, two beam position monitors (BPM), BPM A and BPM B, are installed in Hall A. BPM A is located about $7 \mathrm{~m}$ upstream of the target, BPM B about $1 \mathrm{~m}$. The position of the BPMs is defined with survey. The BPMs are simple cavities with four antennas rotated $\pm 45^{\circ}$ from the horizontal and vertical directions. The signal picked up by each antenna tuned to the fundamental frequency of the beam is inversely proportional to the distance between the beam and the antenna. The beam position can be determined to about $200 \mu \mathrm{m}$ for currents in excess of $10 \mu \mathrm{A}$.

A second method to measure the beam position is using the current of the raster magnets. Because of the energy dependence of the beam bend angle and other nonlinearities the method requires good knowledge of the system. The advantage of the raster method over the BPM method is that no correction for a phase shift is necessary. 


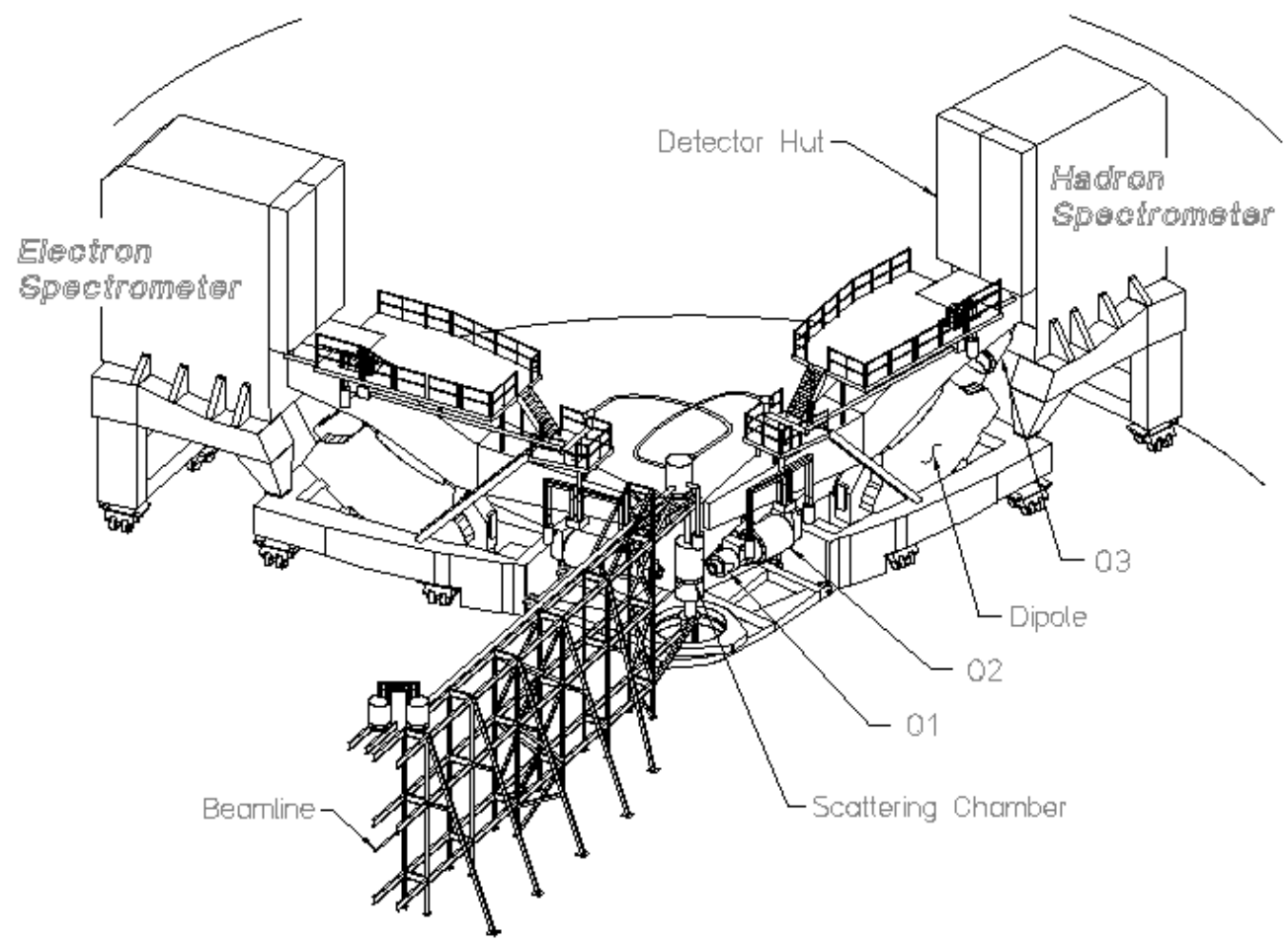

Figure 3.11: Layout of Hall A. Electrons travel through the beamline and impinge on the target mounted in the scattering chamber. The left spectrometer was used to detect electrons, the right spectrometer for the hadrons (protons). The spectrometers are built identically and consist each of three quadrupoles (Q1, Q2, Q3) and a dipole.

\subsubsection{Experimental Setup}

The experiment was done in Hall A. The beam polarization in the Hall can be measured with a Møller polarimeter [60] or a Compton polarimeter [61]. After impinging on the target, the unscattered beam is stopped in the beam dump. Scattered electrons, knockout protons or charged particles produced by the reaction are detected and momentum analyzed by two identical High Resolution Spectrometers (HRS). In this experiment, the left arm (left defined by an observer standing at the target and looking in the direction of the beam dump) HRS was used to detect the scattered electrons; the right arm, equipped with an FPP detected the knockout protons. The following sections will describe the equipment in more detail. Figure 3.11 shows the general layout of Hall A. 


\section{Target}

The high pressure cryogenic target system has three loops: for liquid ${ }^{1} \mathrm{H}$, liquid ${ }^{2} \mathrm{H}$ and gaseous ${ }^{4}$ He. During the experiments two cryogenic targets were used, hydrogen and helium. In addition, a solid carbon target and a beryllium oxide target were used for calibration measurements and beam spot checks. The targets were arranged in a vertical stack and could be easily moved in and out of the beam with the target motion system which was driven by three step-motors and is remotely operated. The liquid hydrogen target was operated at $19 \mathrm{~K}$ and the helium target at $6.3 \mathrm{~K}$. The coolant needed to cool down the gases from room temperature to operating temperature was supplied by the End Station Refrigerator (ESR), which supplied helium coolant at temperatures of either $4.5 \mathrm{~K}$ or $15 \mathrm{~K}$. This means that the coolant supply had to be switched when a change of cryotargets was needed; one cryotarget had to be warmed up and the second target had to be cooled down. This took about eight hours; three changes were needed during the experiment. For the liquid target two horizontal cylindrical cells with lengths of $15 \mathrm{~cm}$ and $4 \mathrm{~cm}$ were available. In this experiment, the $15 \mathrm{~cm}$ cell was used to achieve higher count rates. The helium loop had a single vertical cylindrical cell with a diameter of $10.4 \mathrm{~cm}$. Table 3.3 lists the target parameters during the experiment.

\begin{tabular}{lcc}
\hline \hline & ${ }^{1} \mathrm{H}$ & ${ }^{4} \mathrm{He}$ \\
\hline Target length $(\mathrm{cm})$ & 15 & 10 \\
Temperature $(\mathrm{K})$ & 19 & 4.2 \\
Pressure $(\mathrm{kPa})$ & 17.6 & 150 \\
Density $\left(\mathrm{g} / \mathrm{cm}^{3}\right)$ & 0.07 & 0.13 \\
\hline \hline
\end{tabular}

Table 3.3: Target parameters during the experiment.

\section{High Resolution Spectrometers}

The Hall A HRS are identical magnetic spectrometers consisting of three superconducting quadrupoles (Q) and one superconducting dipole (D) in QQDQ arrangement (as shown in Fig. 3.11). The use of quadrupoles allows to focus in both dispersive and nondispersive directions, therefore increasing the acceptance. 


\begin{tabular}{lcc}
\hline \hline Momentum Range & $\mathrm{GeV}$ & $0.3-4.0$ \\
Configuration & & QQDQ \\
Bend Angle & $\mathrm{deg}$ & 45 \\
Optical Length & $\mathrm{m}$ & 23.4 \\
Momentum Acceptance & $\%$ & \pm 4.5 \\
Dispersion & $\mathrm{cm} / \%$ & 12.4 \\
Radial Linear Magnification & & 2.5 \\
Momentum Resolution FWHM & & $1 \times 10^{-4}$ \\
Angular Acceptance Horizontal & $\mathrm{mr}$ & \pm 28 \\
Angular Acceptance Vertical & $\mathrm{mr}$ & \pm 60 \\
Solid Angle Rectangular & $\mathrm{msr}$ & 6.7 \\
Angular Resolution Horizontal & $\mathrm{mr}$ & 0.6 \\
Angular Resolution Vertical & $\mathrm{mr}$ & 2.0 \\
Transverse Length Acceptance & $\mathrm{cm}$ & $\pm 5 \mathrm{~cm}$ \\
Transverse Position Resolution & $\mathrm{mm}$ & 1.5 \\
Spectrometer Angle Precision & $\mathrm{mr}$ & 0.1 \\
\hline \hline
\end{tabular}

Table 3.4: Table of optical properties for the HRS; numbers taken from [62].

\section{HRS Tracking Detectors}

Each HRS detector stack is equipped with two VDCs, each containing two wire planes. The first VDC is placed close to the optical focus of the HRS, while the second one is offset $50 \mathrm{~cm}$ downstream. This ensures good resolution for the determination of the tracking angle. A special feature of the HRS VDCs is the lack of field wires, which results in a lower operating voltage. The VDC tracking system works very similarly to the system described above; details can be found in [63].

\section{Hadron Arm Detector Stack}

The detector package for the hadron arm including the FPP is shown in Fig. 3.13. It includes the VDCs, scintillators used for timing and trigger, Čerenkov detectors and the FPP. The Hall A FPP consists of a carbon block sandwiched between a set of tracking chambers. The carbon block consists of four "doors" with thicknesses of $22.9 \mathrm{~cm}$, $15.2 \mathrm{~cm}, 7.6 \mathrm{~cm}$ and $3.8 \mathrm{~cm}$, which are operated in remote control. In contrast to the VDCs the tracking chambers are straw chambers, consisting of a set of cylindrical tubes with a thin wire running along the central axis of each tube. The operational 

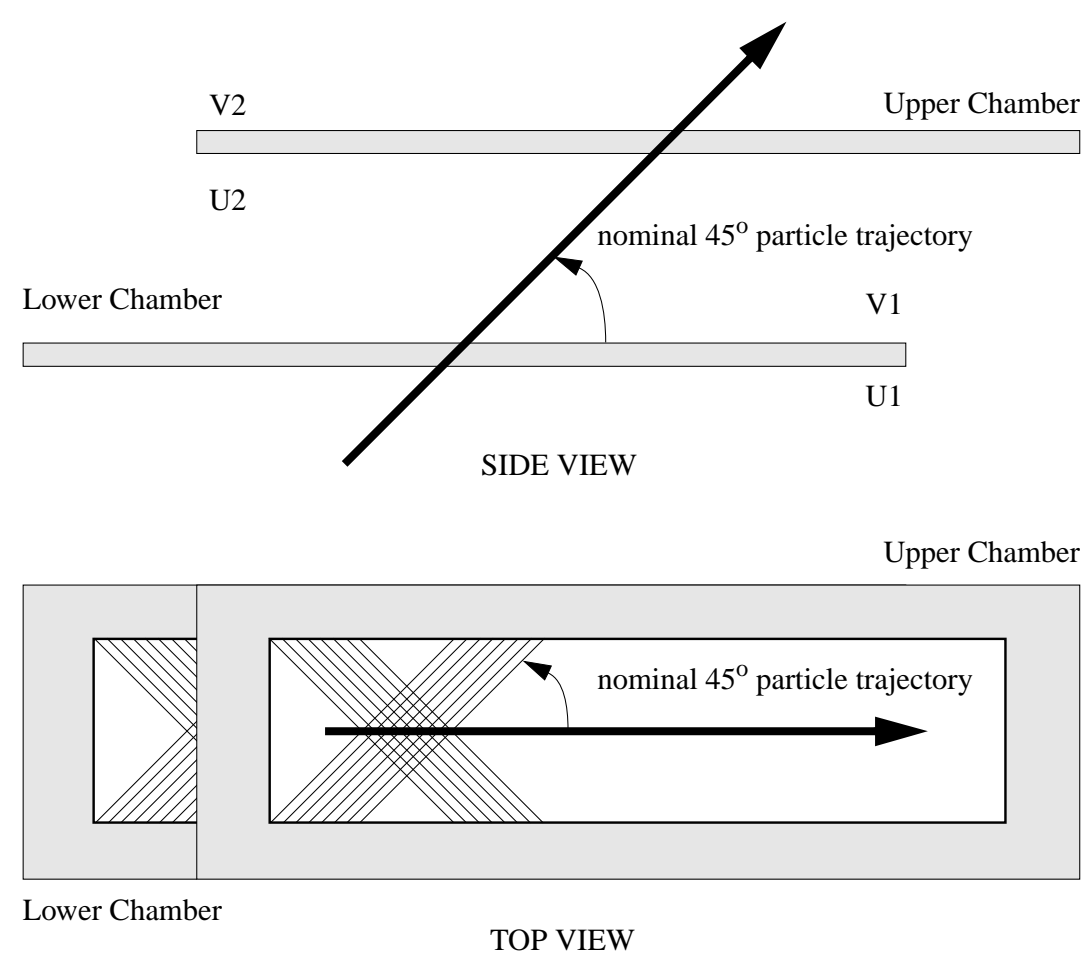

Figure 3.12: Schematic layout of the VDC. Both chambers are tilted $45^{\circ}$ to the central ray. Each VDC consists of two chambers $\mathrm{U}$ and V. V wires are rotated to $90^{\circ}$ relative to U wires. Fig. taken from [63].

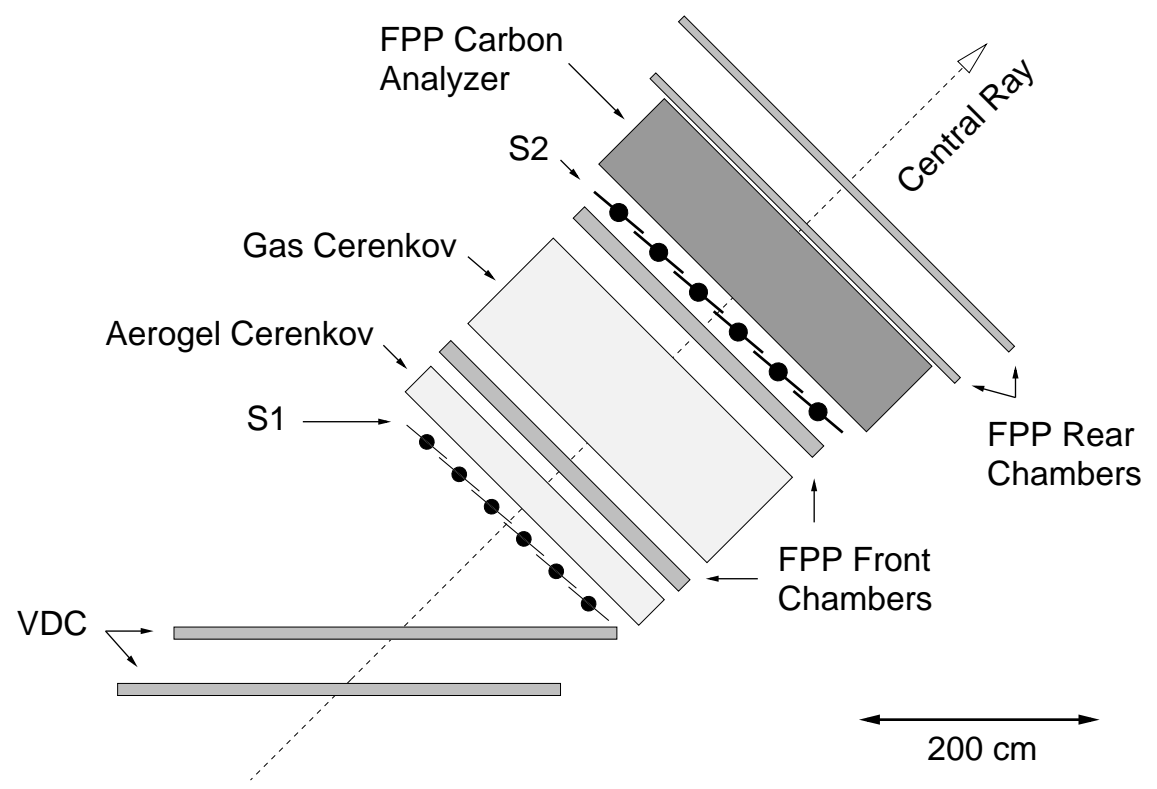

Figure 3.13: Detector package for the hadron arm with vertical drift chambers (VDC), scintillators (S1 and S2), Čerenkov detectors and the FPP (including front and rear chambers and carbon analyzer). The scale is approximate. 
principle of a straw is similar to the HDC, but with single wires. In the FPP, three spatial orientations were used for the straw chambers. $\mathrm{X}$ wires measure position along the dispersive direction; the $\mathrm{U}$ and $\mathrm{V}$ wires are perpendicular to each other and rotated by $45^{\circ}$ with respect to the $\mathrm{X}$ wires. The two front tracking chambers consist each of three $\mathrm{V}$ planes and three $\mathrm{U}$ planes. The first rear chamber after the carbon analyzer has two planes of each $\mathrm{X}, \mathrm{V}$, and $\mathrm{U}$. The second chamber behind the carbon has three $\mathrm{U}$ and three $\mathrm{V}$ planes. For each chamber set, the $\mathrm{U}$ planes are placed closest to the carbon blocks.

\subsubsection{Trigger and Data Acquisition}

The left and right spectrometers have an identical trigger setup which is similar to the setup used in Mainz. The signals from the photomultipliers of the two scintillators are processed by a local Memory Lookup Unit (MLU). A signal pattern which shows a charged particle nearly parallel to the central ray is accepted and generates the single trigger $\mathrm{T} 1$ for the left arm (T3 for the right arm). Using an overlap AND circuit from T1 and T3 produces the coincidence trigger T5. All triggers are then sent to a Trigger Supervising (TS) unit. This unit prescales the single triggers to the preset value. In addition, it checks whether the DAQ is ready and generates the readout. A schematic drawing of the Hall A coincidence trigger taken from [64] is shown in Fig. 3.14. 


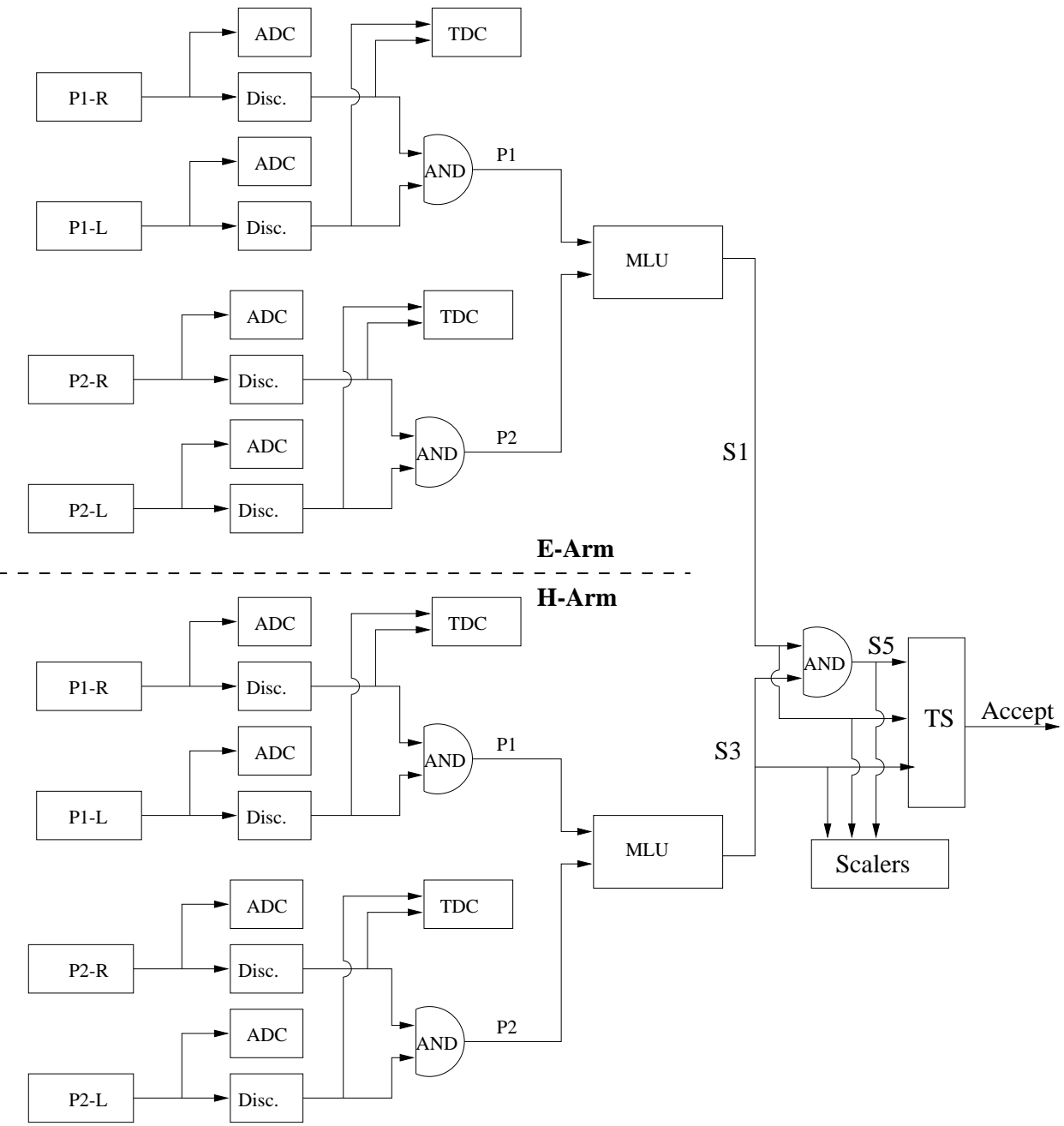

Scintillators

Figure 3.14: Coincidence trigger setup for Hall A. Figure taken from [65]. 


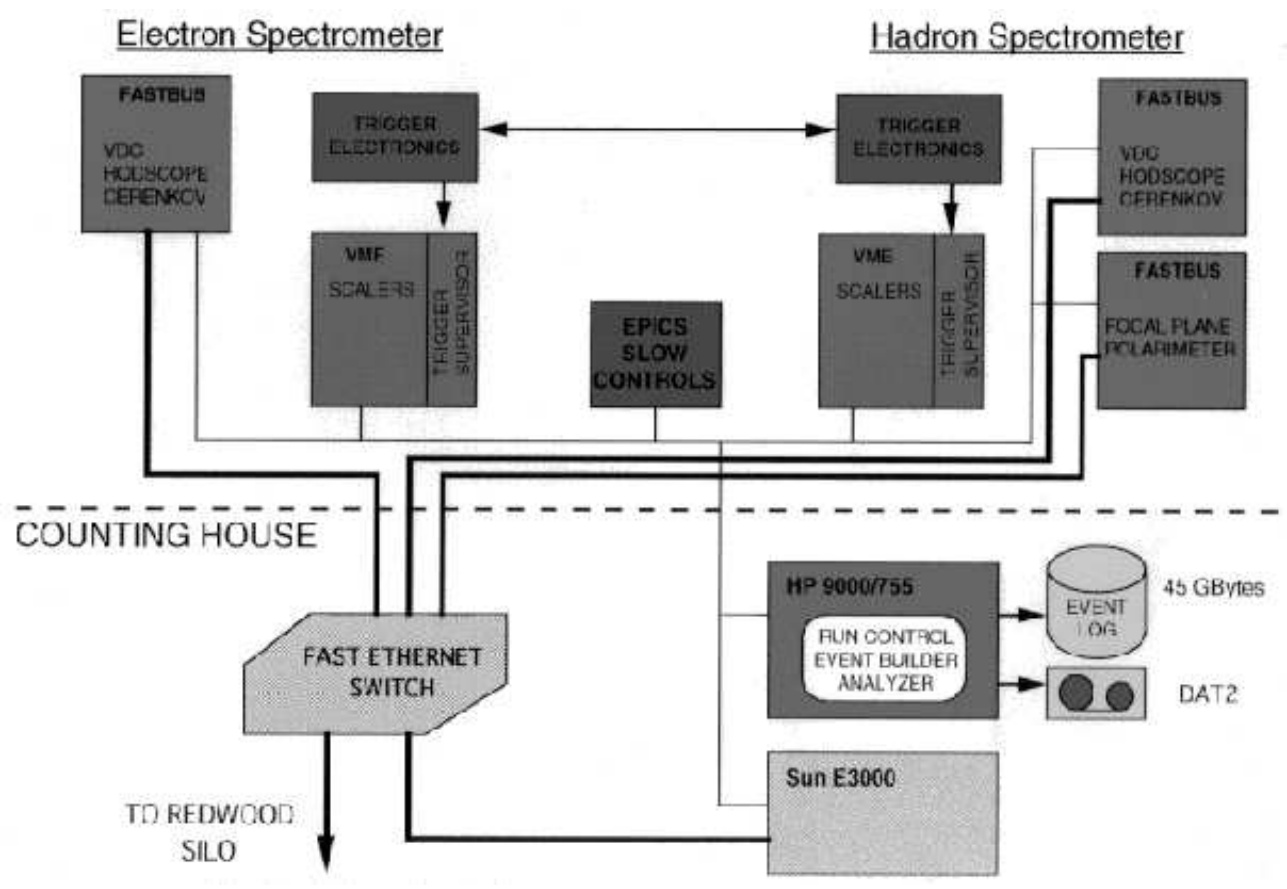

Figure 3.15: Hall A DAQ setup. Figure taken from [64].

The CEBAF Online Data Acquisition (CODA) [66] was used to manage the data acquisition system (Fig. 3.15) during the experiment. The DAQ hardware consists of a setup of VME and Fastbus crates containing various electronic modules and the local TS for each spectrometer. A read-out controller (ROC) handles the communication between CODA and the detectors. The Eventbuilder merges the information from the spectrometers, adds the necessary header information and creates an approximately $1 \mathrm{kB}$ large event. Experimental parameters, e.g. scaler information and different equipment parameters such as target positions were also added to the data stream. In addition, there are monitoring programs (dhist, hana) to provide online display of detector variables during data taking. During each run, the ESPACE ( Event Scanning Program for Hall A Collaboration Experiments) code [67] was used to analyze part of the run to check essential detector performance and quality of the physics data. ESPACE was also used for the offline analysis. 


\section{Chapter 4}

\section{Raw Data Analysis}

\subsection{Introduction}

The following sections explain the data analysis leading from the raw data to physics results. In the first stage of the analysis, the event reconstruction including beam position monitoring, energy loss correction and tracking were done. The next step included calibrations of the spectrometers, detectors, and beam parameters. Finally, good events were selected through software cuts. The analysis approach to the Mainz and JLab data was similar, although differences in the experimental setup sometimes required applying different procedures. These differences will be pointed out explicitly where necessary.

\subsection{Event Reconstruction}

\subsubsection{Data Analysis Codes}

The raw data event, which is mainly information on timing and position, has to be converted to reconstruct the particle's track and calculate its momentum. This was done using the analyzing codes ESPACE (Event Scanning Program for Hall A Collaboration Experiments) at JLab and Cola++ at MAMI.

ESPACE [67] is a FORTRAN based code which can be used to histogram, filter, evaluate conditions and/or accumulate data. Constants of spectrometers and their detectors are stored in a database. A header file contains information which is needed to construct some of the variables, e.g. the target density and thickness, spectrometer positions and offsets, and information about the beam. COLA ++ , based on $\mathrm{C}++$, performs similar functions. 


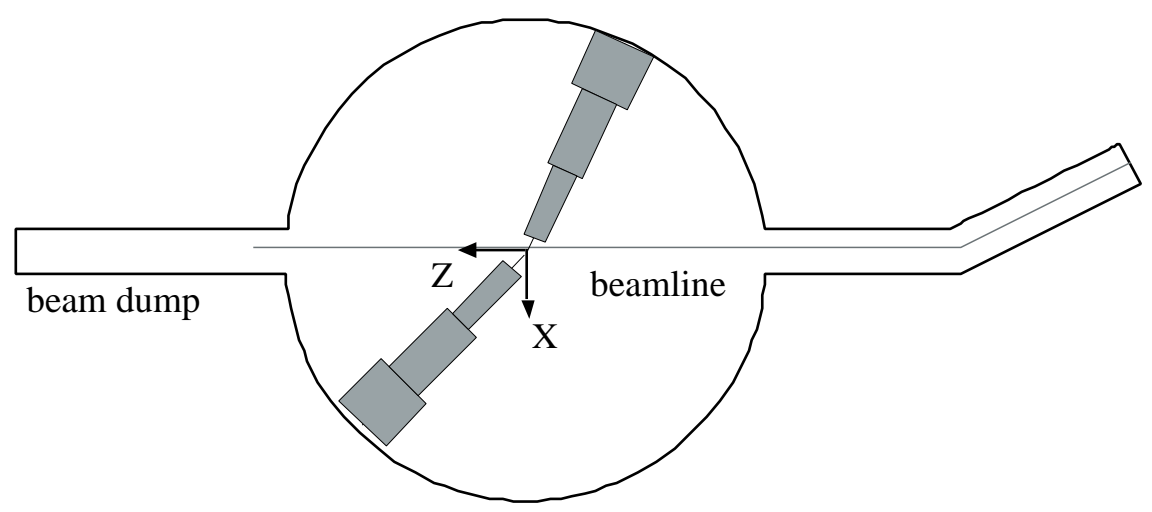

Figure 4.1: Top view of Hall A with the laboratory coordinate system. Figure taken from [67].

\subsubsection{Coordinate Systems}

\section{Laboratory Coordinate System}

The origin of the laboratory coordinate system is defined by the point of closest approach of the non-rastered electron beam and the axis of rotation of the solid target coordinate system. The non-rastered beam direction is defined by the last two beam position monitors. The $z^{\text {lab }}$ coordinate points in the direction of the beam, $y^{\text {lab }}$ is directed upward as defined by gravity, and $x^{\text {lab }}$ completes the right-handed system. Figure 4.1 shows the $x^{\text {lab }}$ and $z^{\text {lab }}$ coordinates of the laboratory coordinate system in Hall A.

\section{Target Coordinate System}

The $z^{\text {tg }}$ direction of this system is perpendicular to the sieve slit surface and going through the midpoint of the central sieve slit hole, pointing away from the target, as shown in Fig. 4.2. The $x^{\text {tg }}$ axis is parallel to the sieve slit surface and points vertically down. The right handed system is completed by $y^{\operatorname{tg}}$. The angle between the beam axis going to the beam dump and the spectrometer mid plane is $\Theta_{0}$. The angles $\phi^{\operatorname{tg}}$ and $\theta^{\operatorname{tg}}$ are measured in the $y z$ and $x z$ plane, respectively.

\section{Focal Plane Coordinate System}

The tracking devices reconstruct the particle track in the focal plane. The coordinates are calculated with respect to the focal plane coordinate system. For JLab, the origin 


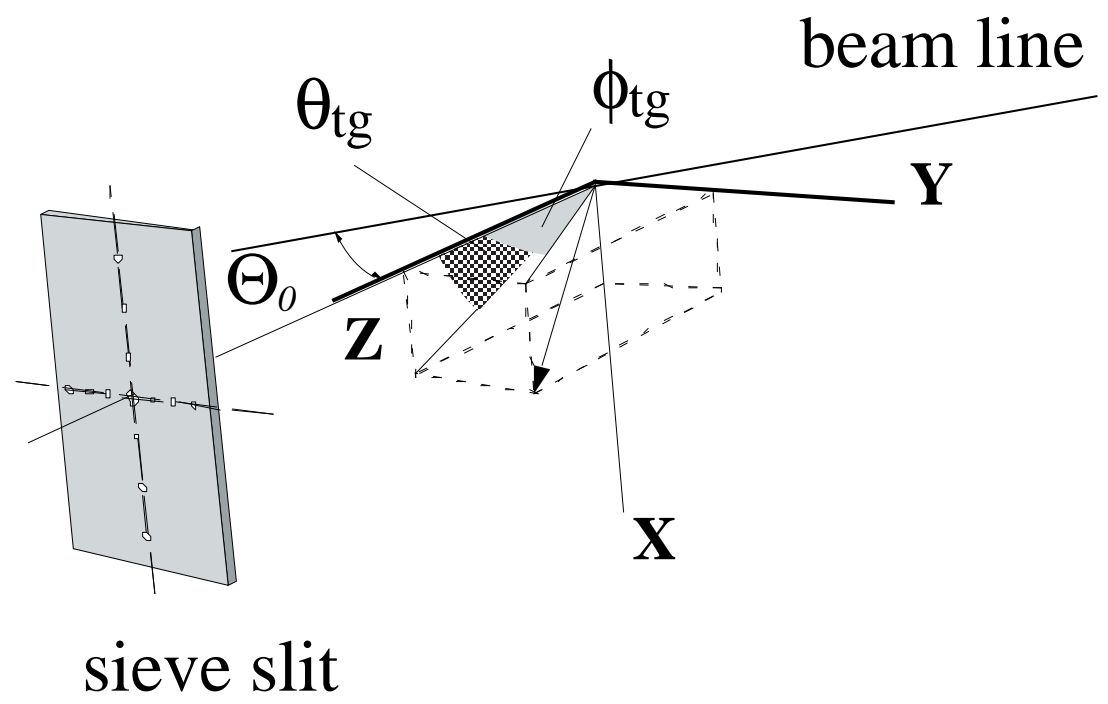

Figure 4.2: Target coordinate system. In this plot, $\theta^{\mathrm{tg}}$ and $\phi^{\mathrm{tg}}$ are both positive. Figure taken from [67].

of this system is defined by the intersection of wire 184 of the VDC U1 plane and the perpendicular projection of wire 184 in the VDC V1 plane onto the VDC U1 plane. The unit vector $\hat{z}^{\mathrm{fp}}$ is pointing along the central ray away from the target, whereas $\hat{x}^{\mathrm{fp}}$ lies in the spectrometer mid-plane pointing away from the center of curvature of the dipole. The angles $\theta^{\mathrm{fp}}$ and $\phi^{\mathrm{fp}}$ are defined as

$$
\begin{aligned}
\tan \theta^{\mathrm{fp}} & =\frac{d x^{\mathrm{fp}}}{d z^{\mathrm{fp}}} \\
\tan \phi^{\mathrm{fp}} & =\frac{d y^{\mathrm{fp}}}{d z^{\mathrm{fp}}}
\end{aligned}
$$

\subsubsection{Tracking}

The physical information from the tracking detectors is the drift time of the charge avalanche, caused by the particle's track, to the signal wire. From these measured drift times, drift distances have to be determined. This depends on the hardware of the tracking detectors and is discussed in detail in e.g. $[65,68]$. Knowing the drift distances and the wire numbers allows for the calculation of the hit position. Once the position is determined, the point and angle of intersection of the particle with the tracking detector chamber is calculated. In principle, the vector $(x, y, \theta, \phi)^{\mathrm{fp}}$ together with the hit position at the chamber is sufficient to determine the track uniquely. However, where several 
tracking detector chambers are involved, e.g. in the JLab VDC detectors, the angles are determined through the hit positions and the distance between the different chambers. The added lever arm results in an improved tracking resolution.

Once the track in the focal plane is established, the transformation to the track at the target is done using the known optical properties of the respective spectrometer. The spectrometers were designed to have a uniquely defined function $\delta=\delta\left(x^{\mathrm{fp}}\right)$. This property is used to express the tracking vector in the target coordinate system as $(\delta, y, \theta, \phi)^{\mathrm{tg}}$. The momentum at the vertex then has to be corrected for the energy loss.

\subsubsection{Energy Loss Correction}

The incident electron up to the reaction vertex and the scattered electron and recoil proton suffer energy losses during their passage through matter. The energy loss in the scattering chamber entry and exit windows, target walls, the target, the air, the spectrometer entry windows and in the scintillators has to be calculated. At JLab, this is done in ESPACE. The target cell model, length of the target and target thickness are entry parameters given in the header file. The energy loss calculation is done in ESPACE, following the equations given in e.g. [69].

In Mainz, the energy loss was treated similarly. In addition to the above mentioned causes for energy loss, humidity from the outside leaked into the target chamber and condensed as so-called "snow" on the outside walls of the cryogenic target. The additional energy loss was corrected in Cola ++ by adding two parameters for snow thickness and density. The data taking in Mainz was done during three periods. In the days between these periods, the target was warmed up. For the change from the helium to hydrogen target and back a target warm-up was also necessary. Each warm-up and subsequent cooldown caused the melting and new condensation of snow. Depending on the outside conditions, the different snow layers had different thickness. These values were set manually in the database and were determined by adjusting the missing energy peak of the respective data taking period to zero. As almost all the energy loss in the target occurs through Coulomb scattering, the effect of energy loss on polarization is expected to be very small. 


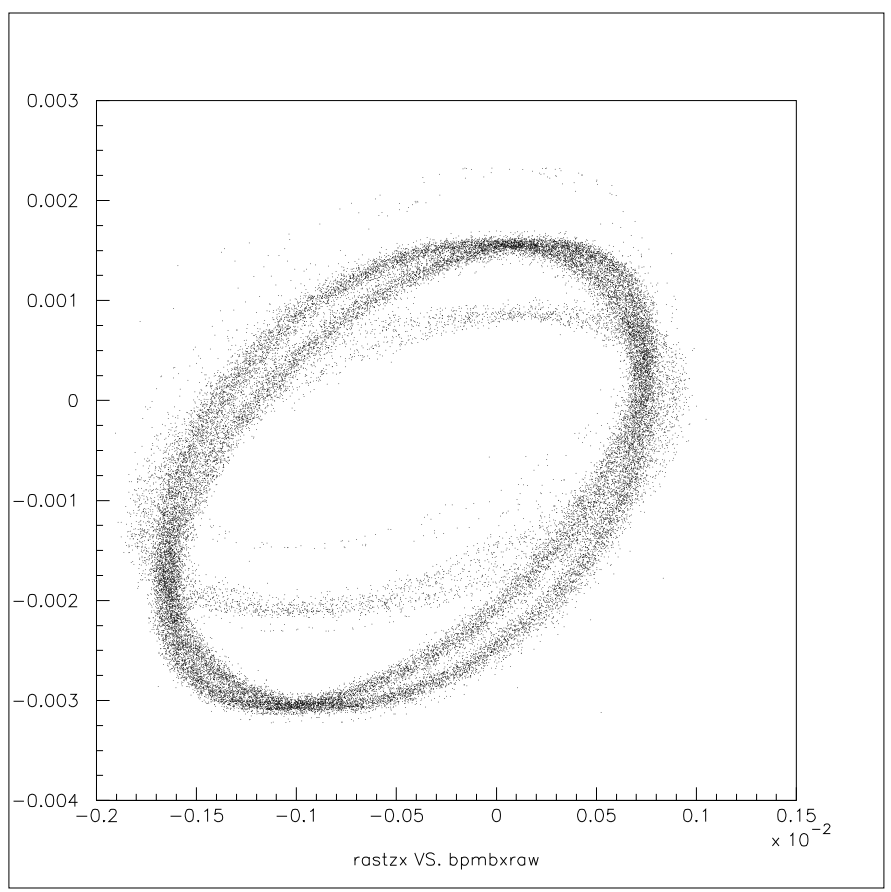

Figure 4.3: Plot of $x_{\mathrm{BPMB}}^{\mathrm{lab}}$ versus $x_{\mathrm{rast}}^{\text {lab }}$ for 30,000 events. The several distinguishable ellipses are a deviation from the norm and hint at a problem in one of the detectors.

\subsubsection{Vertex Reconstruction}

With the information of one spectrometer alone, the $z^{\operatorname{tg}}$ coordinate of the vertex cannot be determined. The knowledge of the beam position and direction on the target together with the track as determined by the spectrometer allows one to calculate the point of intersection between the beam and track vectors and hence $z_{\mathrm{vertex}}^{\mathrm{tg}}$. As discussed in Sec. 4.3.3, two independent systems, the BPMs and the raster monitors, were used to measure the beam position. Their readings were included in the data stream. By analyzing these data, the beam position on the target and its angle with respect to the $z^{\text {lab }}$ direction can be calculated.

Analyzing and plotting the $x^{\text {lab }}$ or $y^{\text {lab }}$ position of the BPMs versus the respective coordinate as given by the raster monitors revealed some irregular behavior. Figure 4.3 shows a two dimensional plot of $x_{\mathrm{BPMB}}^{\text {lab }}$ versus $x_{\text {rast }}^{\text {lab }}$ (by the raster monitor closest to the target) for 5000 events. Normally, one would expect an elliptical event distribution due to the phase difference between the monitoring systems. The multiple ellipses which show up in this plot are a clear sign for problems in one of the detectors. The 
problem was later tracked to a fault in the raster's electronics [70]. Therefore, only the information on the beam position provided by the BPMs was used in the analysis.

\subsection{Calibrations}

\subsubsection{Survey and Spectrometer Alignment}

\section{JLab}

Before and after the experiment in Hall A at JLab, the angle, horizontal (HPT) and vertical (VPT) mispointings of the spectrometers were surveyed with respect to the laboratory coordinate system. In addition, the angle and horizontal mispointing as measured by the linear variable differential transformers (LVDTs) were included in the database. Survey 1 was performed on March 30, a week before the data taking for E93-049 started. The experiment ended on May 8; Survey 2 was performed on May 2nd. The periods between the surveys and data taking were used for maintenance and calibrations. The survey and LVDT results are shown in Table 4.1. It had to be

\begin{tabular}{ll|ccc|cc}
\hline \hline Date & HRS & \multicolumn{3}{|c|}{ Survey } & \multicolumn{2}{c}{ LVDT } \\
& Arm & Angle & HPT & VPT & Angle & HPT \\
& & $($ degree $)$ & $(\mathrm{mm})$ & $(\mathrm{mm})$ & $($ degree $)$ & $(\mathrm{mm})$ \\
\hline 30 Mar 2001 & $\mathrm{L}$ & 20.586 & +1.61 & +0.38 & 20.572 & +3.78 \\
30 Mar 2001 & $\mathrm{R}$ & 25.525 & -3.36 & -0.27 & 25.521 & -2.92 \\
\hline 02 May 2001 & $\mathrm{L}$ & 18.083 & +2.44 & +0.38 & 18.065 & +4.82 \\
02 May 2001 & $\mathrm{R}$ & 47.100 & +1.09 & +0.69 & 47.087 & -1.77 \\
\hline \hline
\end{tabular}

Table 4.1: Survey and LVDT results for the Hall A left and right HRS spectrometers.

decided which survey results should be used for data analysis. The elastic hydrogen data for $Q^{2}=0.5 \mathrm{GeV}^{2}$ and $Q^{2}=1.6 \mathrm{GeV}^{2}$ were used for the investigation, because the reaction is overdetermined. Data taking for $Q^{2}=1.0 \mathrm{GeV}^{2}$ and $Q^{2}=1.6 \mathrm{GeV}^{2}$ was done back-to-back. Because no major alignment shifts were expected to occur during this short time period, and the spectrometers were only moved once during that time, only one data set, at the higher $Q^{2}$, was analyzed. The data were replayed twice using the offsets of the respective surveys. The difference $\delta z^{\text {lab }}=z_{H}^{\text {lab }}-z_{E}^{\text {lab }}$ between the 
reaction vertex coordinate of the electron and hadron arm was plotted. Because the reconstruction of the vertex depends on the survey parameters, $\delta z^{\text {lab }}$ and its full width at half maximum (FWHM) are measures of the reconstruction quality. The result is given in Table 4.2. For all variables, offsets from Survey 1 lead to equal or better values

\begin{tabular}{lcrc}
\hline \hline $\begin{array}{l}Q^{2} \\
(\mathrm{GeV})^{2}\end{array}$ & Survey & $\begin{array}{r}\Delta z^{\text {lab }} \\
(\mathrm{mm})\end{array}$ & $\begin{array}{c}\delta\left(\Delta z^{\text {lab }}\right) \\
(\mathrm{mm})\end{array}$ \\
\hline 0.5 & 1 & -1.31 & 5.09 \\
0.5 & 2 & 1.65 & 5.08 \\
\hline 1.6 & 1 & -0.14 & 3.50 \\
1.6 & 2 & 2.84 & 3.43 \\
\hline \hline
\end{tabular}

Table 4.2: Comparison of surveys using hydrogen data. The difference between the reaction vertex coordinate of the electron and hadron arm is $\Delta z^{\text {lab }}, \delta\left(\Delta z^{\text {lab }}\right)$ is the FWHM of the $\Delta z^{\text {lab }}$ distribution.

and resolutions of the reaction coordinates and thus were chosen for data analysis. A summary of the final spectrometer settings based on Survey 1 is given in Table 4.3.

\section{Mainz}

The Mainz spectrometers are mounted on a support rail with angle markers for digital readout. The angles and positions of the spectrometers are assumed to be known precisely. There is no standard survey procedure in place for checking the positioning before or after an experiment.

\subsubsection{Alignment of Detectors}

\section{JLab VDC alignment}

The VDC alignment is included in the offset optimization of the spectrometer optics. A new set of parameters is only required when the VDC is moved out of position, or the detector stack as a whole is moved. The alignment is done using an elastic or quasi-elastic ${ }^{12} \mathrm{C}\left(e, e^{\prime} p\right)$ data set with the sieve slit in place. The comparison of the actual position of the central sieve slit hole with the nominal position leads to the offset parameters for the positions. The angles $\theta^{\operatorname{tg}}$ and $\phi^{\text {tg }}$ are optimized using the calibrated 


\begin{tabular}{lc|ccccc}
\hline \hline $\begin{array}{l}Q^{2} \\
\left(\mathrm{GeV}^{2}\right)\end{array}$ & Target & $\begin{array}{c}p \\
(\mathrm{MeV})\end{array}$ & $\begin{array}{c}\Theta \\
(\mathrm{deg})\end{array}$ & $\begin{array}{c}x^{\text {off }} \\
(\mathrm{mm})\end{array}$ & $\begin{array}{c}y^{\text {off }} \\
(\mathrm{mm})\end{array}$ & $\begin{array}{c}z^{\text {off }} \\
(\mathrm{mm})\end{array}$ \\
\hline & & \multicolumn{5}{|c}{ Electron Arm } \\
0.5 & $\mathrm{H}$ & 3102.2 & 12.503 & 0.148 & 0.038 & -0.033 \\
0.5 & $\mathrm{He}$ & 3102.2 & 12.469 & 0.149 & 0.038 & -0.033 \\
1.0 & $\mathrm{H}$ & 3666.9 & 14.559 & 0.346 & 0.038 & -0.090 \\
1.0 & $\mathrm{He}$ & 3666.9 & 14.560 & 0.343 & 0.038 & -0.090 \\
1.6 & $\mathrm{H}$ & 3340.3 & 19.352 & 0.289 & 0.038 & -0.102 \\
1.6 & $\mathrm{He}$ & 3340.3 & 19.352 & 0.289 & 0.038 & -0.101 \\
2.6 & $\mathrm{He}$ & 2795.8 & 27.097 & 0.237 & 0.038 & -0.122 \\
\hline & & \multicolumn{5}{|c}{ Hadron Arm } \\
0.5 & $\mathrm{H}$ & 766.0 & 63.120 & -0.029 & 0.027 & -0.058 \\
0.5 & $\mathrm{He}$ & 766.0 & 61.428 & 0.017 & 0.027 & 0.031 \\
1.0 & $\mathrm{H}$ & 1149.6 & 54.819 & 0.095 & 0.027 & 0.134 \\
1.0 & $\mathrm{He}$ & 1149.6 & 54.548 & 0.171 & 0.027 & 0.232 \\
1.6 & $\mathrm{H}$ & 1548.9 & 46.767 & 0.171 & 0.027 & 0.182 \\
1.6 & $\mathrm{He}$ & 1548.9 & 46.767 & 0.162 & 0.027 & 0.166 \\
2.6 & $\mathrm{He}$ & 2160.6 & 36.201 & 0.236 & 0.027 & 0.173 \\
\hline \hline
\end{tabular}

Table 4.3: Spectrometer angles and offsets for the different settings of JLab E93-049 as deduced from spectrometer Survey 1. Spectrometer momenta are taken from NMR readouts.

angles of the non-central sieve slit holes. The coordinate $y^{\text {tg }}$ is optimized by optimizing the reaction position along the beam direction $z^{\text {lab }}$. For doing this, data using a set of foil targets is required. A detailed description of the offset optimization procedure can be found in [71].

\section{JLab FPP Alignment}

After the position of the VDC relative to the central ray of the spectrometer is known, the front and rear FPP chambers have to be aligned relative to the VDC. Such calibration data, where no carbon blocks are placed between the chambers to be aligned, are called straight through data. For the alignment of the front chambers any run which covers the acceptance of the chambers completely can be used for this purpose. To get a good illumination for the alignment of the rear FPP chambers with their large acceptance, a carbon block is placed behind the VDC for the calibration run. There 
are two methods to align the chambers.

In the first method [65], each chamber is aligned separately to the VDC using a set of six parameters, three each for the translational and rotational offsets. For each track, the position and direction as measured by the VDC are projected onto the respective FPP chamber. For the differences between the FPP and VDC values, a $\chi^{2}$ minimization is performed. The corrected values for each chamber are then used as input in the tracking routine. The advantage of this method is the direct link between the alignment parameters and the physical translation and rotation of the chambers. Second and higher order corrections, which are necessary when the chambers are not perfectly planar, are not yet implemented in this method. Such chamber distortions could be caused by the weight of the chambers, distorting them in places far away from the three support points.

In the second method [72], it is assumed that the front and rear chambers are internally aligned. The alignment to the VDC is done by aligning the already reconstructed front chamber track to the VDC track; the same applies to the alignment of the rear chambers to the front chambers. The alignment parameters obtained with this method are not easily related to physical offsets or rotations. On the other hand, extension to higher order corrections is straightforward and was developed for analyzing E93-049.

Comparative studies have shown that both alignment methods lead to equally good results for the precision required by previous experiments. However, the extremely high precision data available in E93-049 showed that using the second method and extending it to higher orders is necessary, especially if one aims to extract induced polarizations.

\section{Alignment Procedure}

A track can be described in the FPP coordinate system by four parameters $x_{0}, y_{0}$, $\theta_{0}$, and $\phi_{0}$, where the subscript 0 indicates that the parameters were taken at $z_{\text {close }}$ values indicated below, where $z_{\text {close }}$ is defined as the distance between the $z$ coordinate of closest approach between the front and rear track to the origin $z=0$. This $z_{\text {close }}$ value was chosen such that the width of the difference between the VDC and FPP track coordinate distributions were minimized. The front and VDC track parameters 
are related by

$$
\left(\begin{array}{l}
x_{0} \\
y_{0} \\
\theta_{0} \\
\phi_{0}
\end{array}\right)_{\text {align }}^{\text {front }}=\left(\begin{array}{c}
x_{0} \\
y_{0} \\
\theta_{0} \\
\phi_{0}
\end{array}\right)_{\text {raw }}^{\text {front }}+\mathcal{A}^{\text {front }}+\mathcal{B}^{\text {front }} \cdot\left(\begin{array}{c}
x_{0} \\
y_{0} \\
x_{0}^{2} \\
y_{0}^{2} \\
x_{0} y_{0}
\end{array}\right)_{\text {raw }}^{\text {front }} .
$$

The elements of the matrices $\mathcal{A}^{\text {front }}$ and $\mathcal{B}^{\text {front }}$ are determined by a least squares fit to straight-through data at $z_{\text {close }}=100 \mathrm{~cm}$. If for any reason only the VDC is moved during a detector access, but none of the FPP chambers, only the front to VDC alignment is necessary. The alignment of the rear to the front FPP is therefore done in two steps. In the first step, the new rear track is defined as

$$
\left(\begin{array}{l}
x_{0} \\
y_{0} \\
\theta_{0} \\
\phi_{0}
\end{array}\right)_{\text {new }}^{\text {rear }}=\left(\begin{array}{l}
x_{0} \\
y_{0} \\
\theta_{0} \\
\phi_{0}
\end{array}\right)_{\text {raw }}^{\text {rear }}+\mathcal{A}^{\text {front }}
$$

which means that now, in addition to the front chamber alignment, a misalignment of the whole FPP tracking detector package to the VDC is already corrected. The second step is the alignment of the rear chamber to the front chamber, which is done similarly to the front-to-VDC alignment:

$$
\left(\begin{array}{l}
x_{0} \\
y_{0} \\
\theta_{0} \\
\phi_{0}
\end{array}\right)_{\text {align }}^{\text {rear }}=\left(\begin{array}{c}
x_{0} \\
y_{0} \\
\theta_{0} \\
\phi_{0}
\end{array}\right)_{\text {new }}^{\text {rear }}+\mathcal{A}^{\text {rear }}+\mathcal{B}^{\text {rear }} \cdot\left(\begin{array}{c}
x_{0} \\
y_{0} \\
x_{0}^{2} \\
y_{0}^{2} \\
x_{0} y_{0}
\end{array}\right)_{\text {new }}^{\text {rear }}
$$

The coefficients are extracted by a least-square fit to calibration data at $z_{\text {close }}=360 \mathrm{~cm}$. Plotting $z_{\text {close }}$, which is defined as the $z$-coordinate for the closest approach of front and rear tracks in the focal plane coordinate system, shows clearly the success of the alignment. Figure 4.4 (a) shows the unaligned $z_{\text {close }}$ spectrum for a measurement with the $7.6 \mathrm{~cm}$ and $3.8 \mathrm{~cm}$ carbon doors in. Figure 4.4 (b) shows the same data set after 

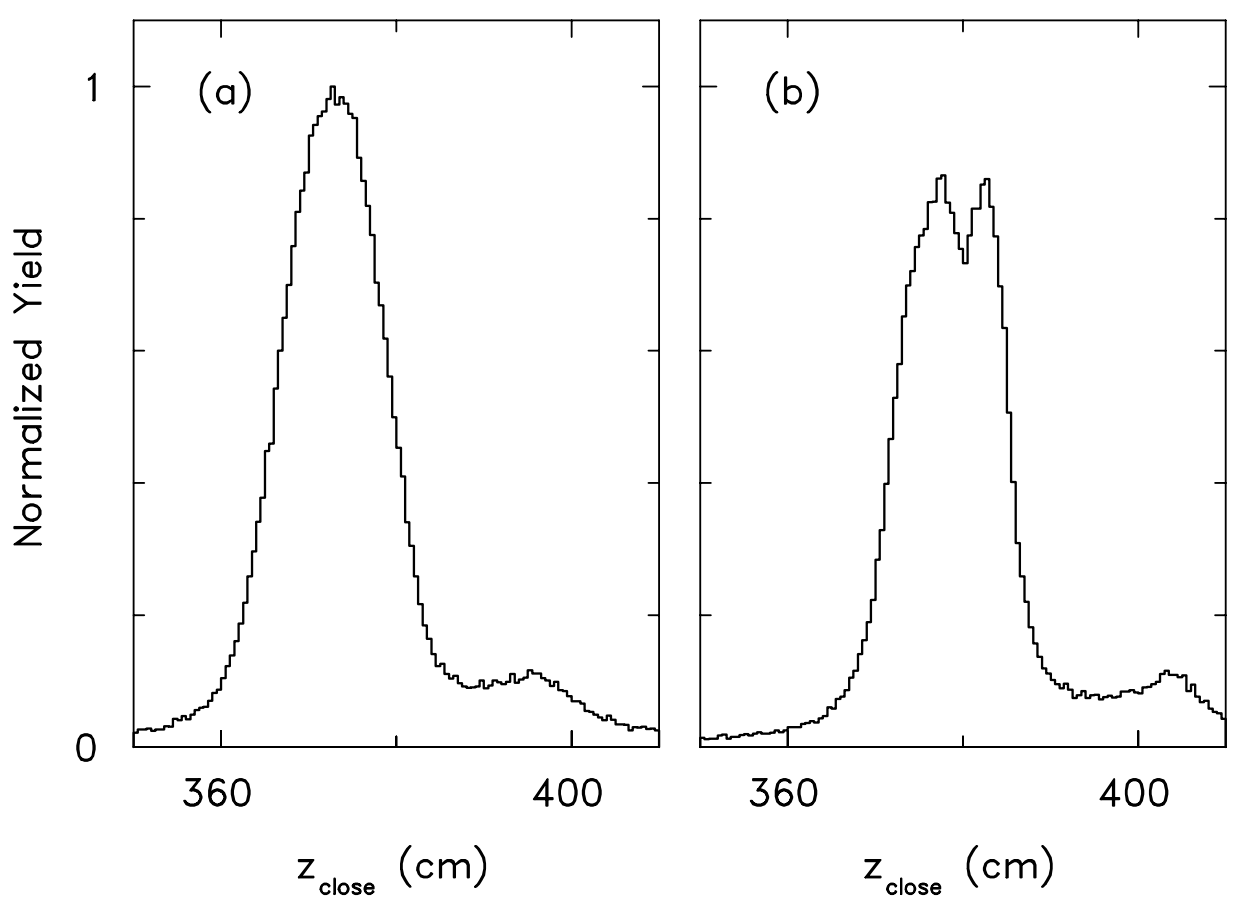

Figure 4.4: Comparison of $z_{\text {close }}$ before (a) and after (b) the FPP chamber alignment. Data taken from ${ }^{1} \mathrm{H}\left(\vec{e}, e^{\prime} \vec{p}\right)$ at $Q^{2}=0.5 \mathrm{GeV}^{2}$ with the $7.6 \mathrm{~cm}$ and $3.8 \mathrm{~cm}$ carbon doors in. The peak at $z_{\text {close }}=395 \mathrm{~cm}$ is caused by protons scattered from the first rear FPP chamber.

the chambers were aligned. The gaps of about $1 \mathrm{~cm}$ between the carbon doors can now be clearly seen. Also the slopes at the beginning and the end of the carbon blocks are steeper.

\section{Mainz VDC Alignment}

The particle tracking performance of the spectrometer A and B detectors depends on the knowledge of the position and orientation of the respective VDC detector package, which is given in terms of $x^{\mathrm{fp}}, y^{\mathrm{fp}}, \theta^{\mathrm{fp}}$ and $\phi^{\mathrm{fp}}$ offset parameters. To determine these parameters, the elastic ep scattering data were used in the following method.

With the assumption that the beam energy is known, only one other experimental observable is needed to calculate all other kinematic parameters of the elastic ep kinematics. So using either one of $p_{e}, p_{p}, \Theta_{e}$, or $\Theta_{p}$ the other three variables were calculated and subtracted from the experimental value on an event-by-event basis. E.g., in the bottom row, leftmost graph of Fig. 4.5, the experimental proton angle $\Theta_{p}$ was used together with the known beam energy to calculate $p_{e}$. Plotted is the difference 


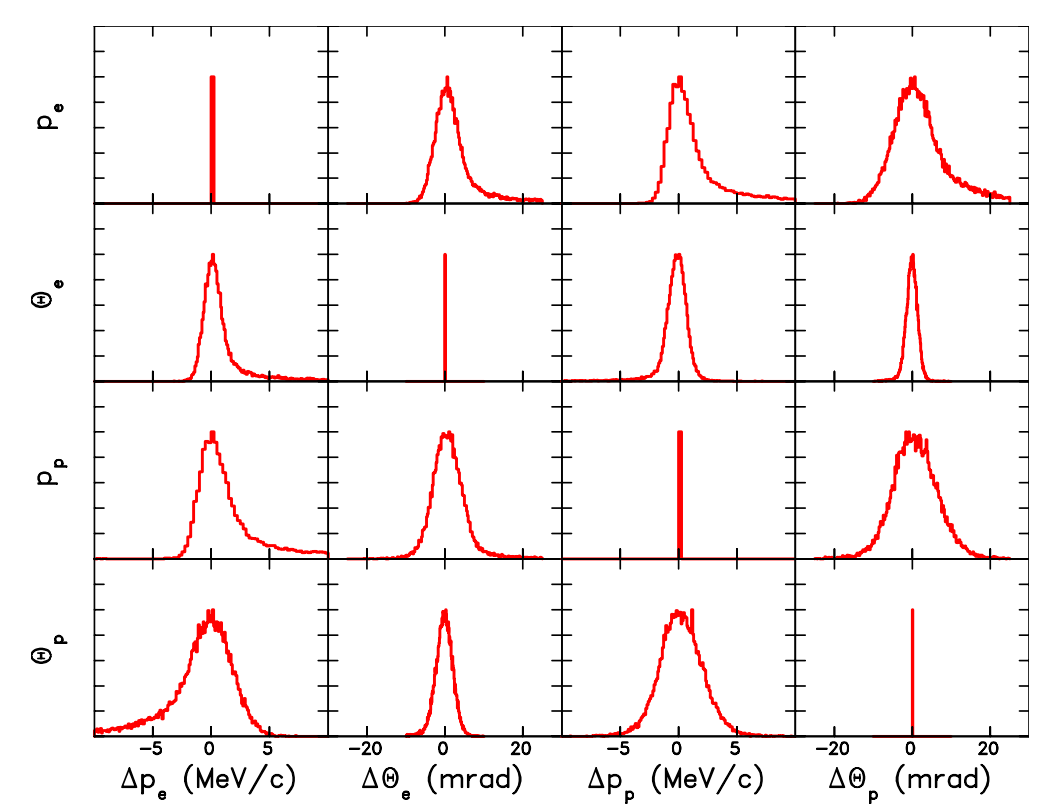

Figure 4.5: Event distributions from elastic ep scattering used for pointing checks; see text for details.

$\Delta p_{e}=p_{e}^{\exp }-p_{e}^{\text {calc }}$. For the unaligned VDCs, the maximum of these distributions were shifted away from zero, as would be expected for an aligned system. To align the VDCs, the offset parameters were adjusted in such a way that the maxima of the $\Delta$ distributions were close to zero. Fig. 4.5 shows the distributions after the VDC alignment. The non-Gaussian part of the distributions stems from the radiative tail of the scattered electron. The solution was cross-checked by using the adjusted offsets from Table 4.4 in the analysis of data taken with a thin carbon target. The resulting target position was $1 \mathrm{~mm}$ upstream from the center of the scattering chamber, which agrees with a survey of the carbon target immediately before the experiment.

\begin{tabular}{lcc}
\hline \hline & Spec. A & Spec. B \\
\hline$x^{\mathrm{fp}}(\mathrm{mm})$ & 46.7 & 21.0 \\
$y^{\mathrm{fp}}(\mathrm{mm})$ & -3.934 & 1.183 \\
$\phi^{\mathrm{fp}}(\mathrm{mrad})$ & 0.154 & -3.01 \\
$\theta^{\mathrm{fp}}(\mathrm{mrad})$ & 786.03 & 839.03 \\
\hline \hline
\end{tabular}

Table 4.4: Adjusted offset parameters for the VDC detector packages of the Mainz spectrometers. 


\section{Mainz FPP Alignment}

The first step in the HDC calibration is the adjustment of the odd-even offsets. To do this, the odd-even spectra for each wire are plotted. These spectra show how many times and at which distance an event was registered to the left or right of the respective wire. The offsets are then corrected to center these spectra symmetrically around zero. To obtain the correct drift time, the single wire TDC offsets had to be determined. This is done by checking the single wire timing spectra and adjusting their offsets. The last step is the overall chamber alignment using straight through data. The reconstructed focal plane coordinates of the HDC are compared to the VDC tracks. Of the six coordinates necessary to describe the position, only the two positions $x$ and $y$ and the two angles $\theta$ and $\phi$ are corrected. This is done by plotting the difference between the respective VDC and HDC variable and adjusting the offset in a way that the mean of the distribution is at zero. Table 4.5 lists the offsets for this experiment.

\begin{tabular}{lccc}
\hline \hline $\begin{array}{l}x_{\text {off }} \\
(\mathrm{mm})\end{array}$ & $\begin{array}{c}y_{\text {off }} \\
(\mathrm{mm})\end{array}$ & $\begin{array}{c}\theta_{\text {off }} \\
(\mathrm{mrad})\end{array}$ & $\begin{array}{c}\phi_{\text {off }} \\
(\mathrm{mrad})\end{array}$ \\
\hline 783.675 & -0.05 & 10.279 & 617.052 \\
\hline \hline
\end{tabular}

Table 4.5: HDC offsets for Mainz.

\subsubsection{Beam Energy and Polarization}

\section{JLab}

For $Q^{2}=0.5 \mathrm{GeV}^{2}$, a lower beam energy was used than for the other settings. For each energy an $e p$ and an ARC measurement was made. The results of these measurements are given in Table 4.6. The lower beam energy falls within the region where ep results are systematically lower than ARC results. To get a better precision on the beam energy and to study the beam energy change during the experiment, the hydrogen data were analyzed with the energies as measured by the ARC method. With the assumption that $p_{e}$ and $p_{p}$ are accurately determined by the spectrometers, and the energy loss in the target known through energy loss calculations, $E_{\text {calc }}=E\left(p_{e}, p_{p}\right)$ was calculated. 


\begin{tabular}{lccc}
\hline \hline Date & Method & Energy (MeV) & Uncertainty (MeV) \\
\hline 8 Apr 2000 & ARC & 3401.160 & 0.600 \\
9 Apr 2000 & ep & 3397.649 & 0.200 \\
4 May 2000 & ep & 4239.406 & 0.250 \\
5 May 2000 & ARC & 4237.500 & 2.000 \\
\hline \hline
\end{tabular}

Table 4.6: Results of the beam energy measurements in MeV during data taking of E93-049.

The $\Delta E=E_{\text {calc }}-E_{\mathrm{ARC}}$ spectrum was then plotted for each $Q^{2}$ point. The peak of the $\Delta E$ distribution was then shifted to $\Delta E=0$. The beam energies corrected by these energy offsets are given in Table 4.7. The energy difference between the data taken at $Q^{2}=1.0 \mathrm{GeV}^{2}$ and the higher $Q^{2}$ data, although they were nominally taken at the same beam energy, may have two causes. The spectrometer constants used in the database change by $1 \cdot 10^{-4}$ over the momentum range of the spectrometer. This can account for up to $0.3 \mathrm{MeV}$ of the difference caused by the beam energy measurement. Also the energy lock of the accelerator was off several times during the experiment, which can lead to beam energy variations of $<2 \mathrm{MeV}$.

\begin{tabular}{lcccc}
\hline \hline$Q^{2}\left(\mathrm{GeV}^{2}\right)$ & 0.5 & 1.0 & 1.6 & 2.6 \\
\hline$E_{0}(\mathrm{MeV})$ & 3399.9 & 4239.0 & 4237.3 & 4237.3 \\
\hline \hline
\end{tabular}

Table 4.7: Corrected beam energy used for analyzing E93-049.

The beam polarization in Hall A was measured with the Møller polarimeter. Table 4.8 shows the results of these measurements. The lower polarization at $Q^{2}=$ $0.5 \mathrm{GeV}^{2}$ originates in tuning of the accelerator, which was optimized for another hall, resulting in a slightly lower than maximally achievable beam polarization for Hall A. The sign change originates in different positions of the $\lambda / 2$ plate in the path of the laser beam. It cancels for polarization transfer ratios; for single polarizations however it was taken into account. 


\begin{tabular}{lc}
\hline \hline Date & Polarization (\%) \\
\hline $04 / 10$ & $64.7 \pm 0.2$ \\
$04 / 16$ & $66.6 \pm 0.2$ \\
$04 / 19$ & $-67.5 \pm 0.2$ \\
$04 / 21$ & $68.1 \pm 0.2$ \\
$04 / 23$ & $-66.2 \pm 0.2$ \\
$04 / 26$ & $65.3 \pm 0.2$ \\
$04 / 30$ & $-67.6 \pm 0.2$ \\
$04 / 30$ & $-68.4 \pm 0.2$ \\
$05 / 03$ & $70.5 \pm 0.2$ \\
$05 / 05$ & $-70.7 \pm 0.2$ \\
$05 / 10$ & $70.8 \pm 0.2$ \\
\hline \hline
\end{tabular}

Table 4.8: Electron beam polarization as measured by the Hall A Møller polarimeter during E93-049.

\section{Mainz}

The microtron technique with its high number of beam recirculations facilitates a precise energy measurement in the accelerator. Given that the fields of the recirculation magnets are known precisely, and using the absolute energy calibration of the accelerator from [73], the MAMI beam energy can be calculated by [41]:

$$
E(n)=\left[180.02+7.504 \cdot n-3.5 \cdot 10^{-5} \cdot n^{2} \pm 0.16\right] \mathrm{MeV} .
$$

The number of recirculations is $n$; the empirical term in $n^{2}$ accounts for the fringe fields of the recirculation dipoles. The good determination of the beam energy through the accelerator makes additional energy measurements in the hall unnecessary.

At the time of the data taking, the Møller polarimeter in Hall A1 was still under construction. Therefore, no direct method to measure the beam polarization in the hall was available. The polarization was determined at the polarized electron source. However, there is an indirect method to obtain the polarization using the ratio $G_{E}^{p} / G_{M}^{p}$ of ${ }^{1} \mathrm{H}\left(\vec{e}, e^{\prime} \vec{p}\right)$, which does not require the knowledge of the beam polarization. Assuming that $\mu G_{E}^{p} / G_{M}^{p}$ and the carbon analyzing power are well known from world data in the $Q^{2}$ range accessible with MAMI, the average beam polarization can be calculated. Within the error bars this method agrees well with the polarization values from the source. 


\subsection{Event Selection}

For both data analyses, several software cuts were made to ensure good quality of the data. The first set of cuts was applied to ensure good tracking. For the VDCs it was required that the multiplicities, i.e., the number of wires which fired during an event, is between 2 and 10 for all four chambers. The angle between the measured track and the central ray had to be smaller than $22.5^{\circ}$ to remove the extreme tracks from the data. An accepted track in the FPP chambers had to have a number of total hits between 2 and 50, with at least one cluster in one of the $\mathrm{U}$ and $\mathrm{V}$ planes of each chamber.

To exclude all events out of the nominal spectrometer acceptances, cuts were made on the momentum acceptance $\delta p$ and the horizontal and vertical angles $\theta^{\text {tg }}$ and $\phi^{\operatorname{tg}}$. Events coming from the target walls were eliminated by cutting on the $y^{\text {tg }}$ coordinate. For the helium measurements at JLab, the condition $\left|p_{\text {miss }}\right| \leq 240 \mathrm{MeV}$ was applied. All cuts mentioned are independent of the kinematics, and are the same for both HRS spectrometers. Table 4.9 lists the acceptance cuts for the spectrometers at Mainz and JLab. Apart from the acceptance cuts, other cuts dependent on the respective

\begin{tabular}{l|cc|cc}
\hline \hline Variable & \multicolumn{2}{|c|}{ Mainz } & \multicolumn{2}{c}{ JLab } \\
& $\min$ & $\max$ & $\min$ & $\max$ \\
\hline$\theta_{p}^{\mathrm{tg}}(\mathrm{mrad})$ & -70 & 70 & -65 & 65 \\
$\phi_{p}^{\operatorname{tg}}(\mathrm{mrad})$ & -100 & 100 & -32 & 32 \\
$y_{p}^{\operatorname{tg}}(\mathrm{mm})$ & -25 & 25 & -65 & 65 \\
$\theta_{e}^{\operatorname{tg}}(\mathrm{mrad})$ & -70 & 70 & -65 & 65 \\
$\phi_{e}^{\mathrm{tg}}(\mathrm{mrad})$ & -20 & 20 & -32 & 32 \\
$y_{e}^{\operatorname{tg}}(\mathrm{mm})$ & -25 & 25 & -65 & 65 \\
$\delta p_{e}(\%)$ & -7.5 & 7.5 & -4.5 & 4.5 \\
$\delta p_{p}(\%)$ & -5.0 & 15.0 & -4.5 & 4.5 \\
\hline \hline
\end{tabular}

Table 4.9: Software cuts related to the acceptance of spectrometers at Mainz and JLab. These cuts are independent of the respective kinematics.

kinematics and targets were used. Cutting on $p_{e}$ ensured that few events from the radiative tail of the electron were analyzed. As shown in Table 4.10, the limits are slightly different for helium and hydrogen measurements at the same $Q^{2}$ due to the recoil momentum in ${ }^{4} \mathrm{He}\left(\vec{e}, e^{\prime} \vec{p}\right)^{3} \mathrm{H}$. These cuts were not applied for the Mainz data. 


\begin{tabular}{lccc}
\hline \hline $\begin{array}{l}Q^{2} \\
\left(\mathrm{GeV}^{2}\right)\end{array}$ & Target & $\begin{array}{c}p_{e}^{\min } \\
(\mathrm{MeV})\end{array}$ & $\begin{array}{c}p_{e}^{\max } \\
(\mathrm{MeV})\end{array}$ \\
\hline 0.5 & $\mathrm{H}$ & 3080 & 3155 \\
0.5 & $\mathrm{He}$ & 3050 & 3125 \\
1.0 & $\mathrm{H}$ & 3520 & 3875 \\
1.0 & $\mathrm{He}$ & 3595 & 3715 \\
1.6 & $\mathrm{H}$ & 3300 & 3430 \\
1.6 & $\mathrm{He}$ & 3015 & 3410 \\
2.6 & $\mathrm{He}$ & 2650 & 2900 \\
\hline \hline
\end{tabular}

Table 4.10: Software cuts on the electron momentum (in MeV) for JLab kinematics.

The event selections mentioned above concerned only single spectrometer events. The next level of cuts concerned the coincidence. Only events were analyzed for which both spectrometer tracking detectors had fired. The timing of the signals had to be within a given coincidence time window. Figure 4.6 shows the measured coincidence time; the zero value is chosen arbitrarily. The prominent peak between the dashed vertical bars are true coincidence events. All other events are accidental coincidences and were not analyzed. The peaks within these accidental coincidences originate in the microstructure of the beam. It should be noted that the background due to the remaining accidental coincidences below the true coincidence peak is very low. The last cut, which was only applied for the helium data, selected the reaction of interest ${ }^{4} \mathrm{He}\left(\vec{e}, e^{\prime} \vec{p}\right)^{3} \mathrm{H}$. In addition, to make sure only the one-body breakup reaction data was analyzed, $18 \mathrm{MeV} \leq E_{\text {miss }} \leq 26 \mathrm{MeV}$ was required

Figure 4.7 demonstrates the effectiveness of the software cuts to reduce background. The missing mass distributions for ${ }^{4} \mathrm{He}\left(\vec{e}, e^{\prime} \vec{p}\right)^{3} \mathrm{H}$ taken in Mainz are plotted in semi$\log$ scale, where $m_{\text {miss }}=0$ represents the tritium ground state. Panel (a) shows the missing mass distribution before any cuts were applied. The hydrogen from the snow on the target walls appears as background at $m_{\text {miss }}=-19 \mathrm{MeV}$. The structure at $m_{\text {miss }}=6 \mathrm{MeV}$ corresponds to the threshold of the ${ }^{4}$ He two-body breakup. Panel (b) shows the same data set after making software cuts, excluding the cuts on missing mass and cuts concerning the FPP. The background is greatly reduced. 


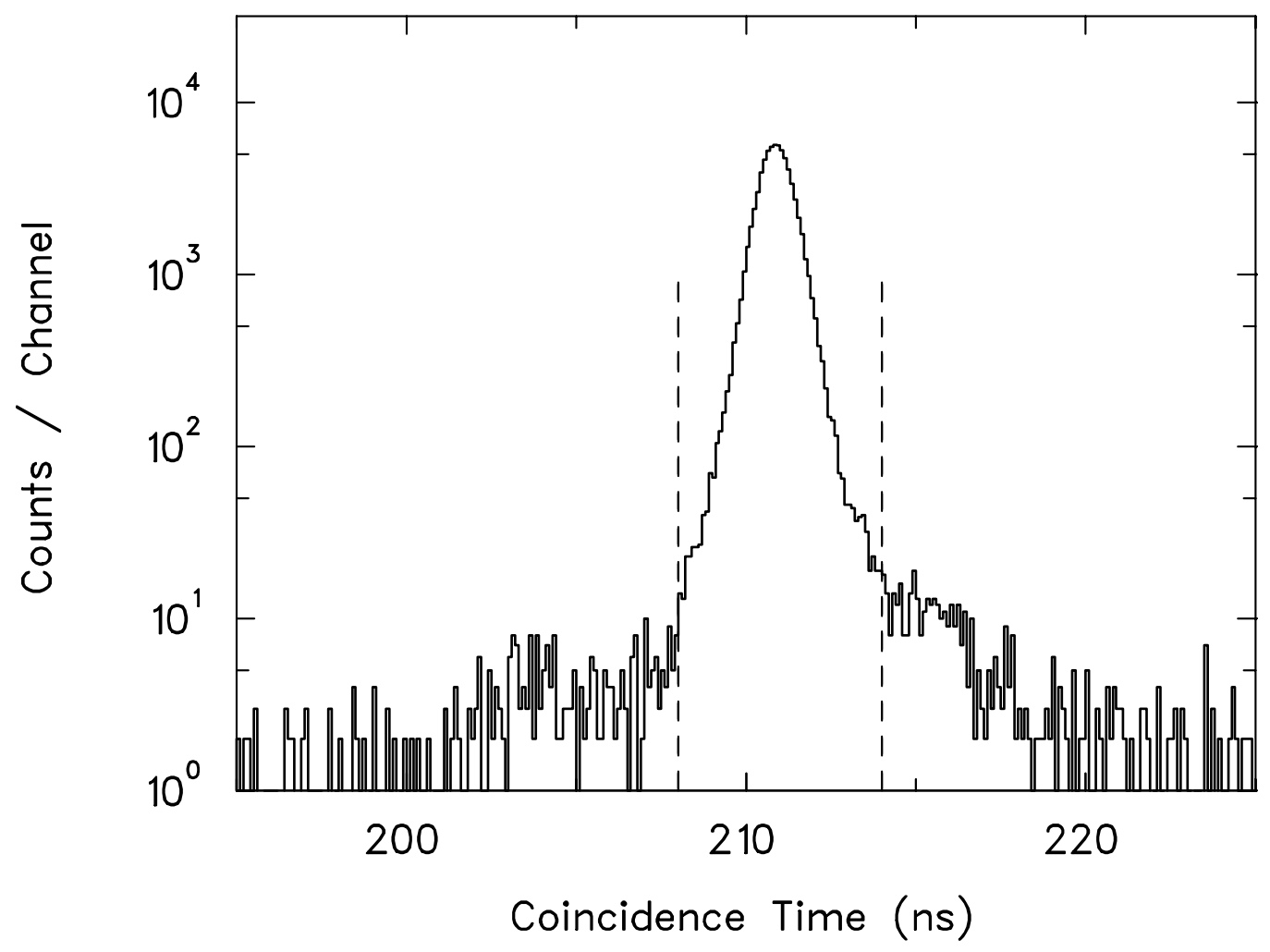

Figure 4.6: Coincidence time for ${ }^{1} \mathrm{H}\left(\vec{e}, e^{\prime} \vec{p}\right)$ at $Q^{2}=0.5 \mathrm{GeV}^{2}$. The lower and upper limit of the cut is indicated by the dashed vertical bars. The peaks to the left and right of the true coincidences originate in the microstructure of the beam. 

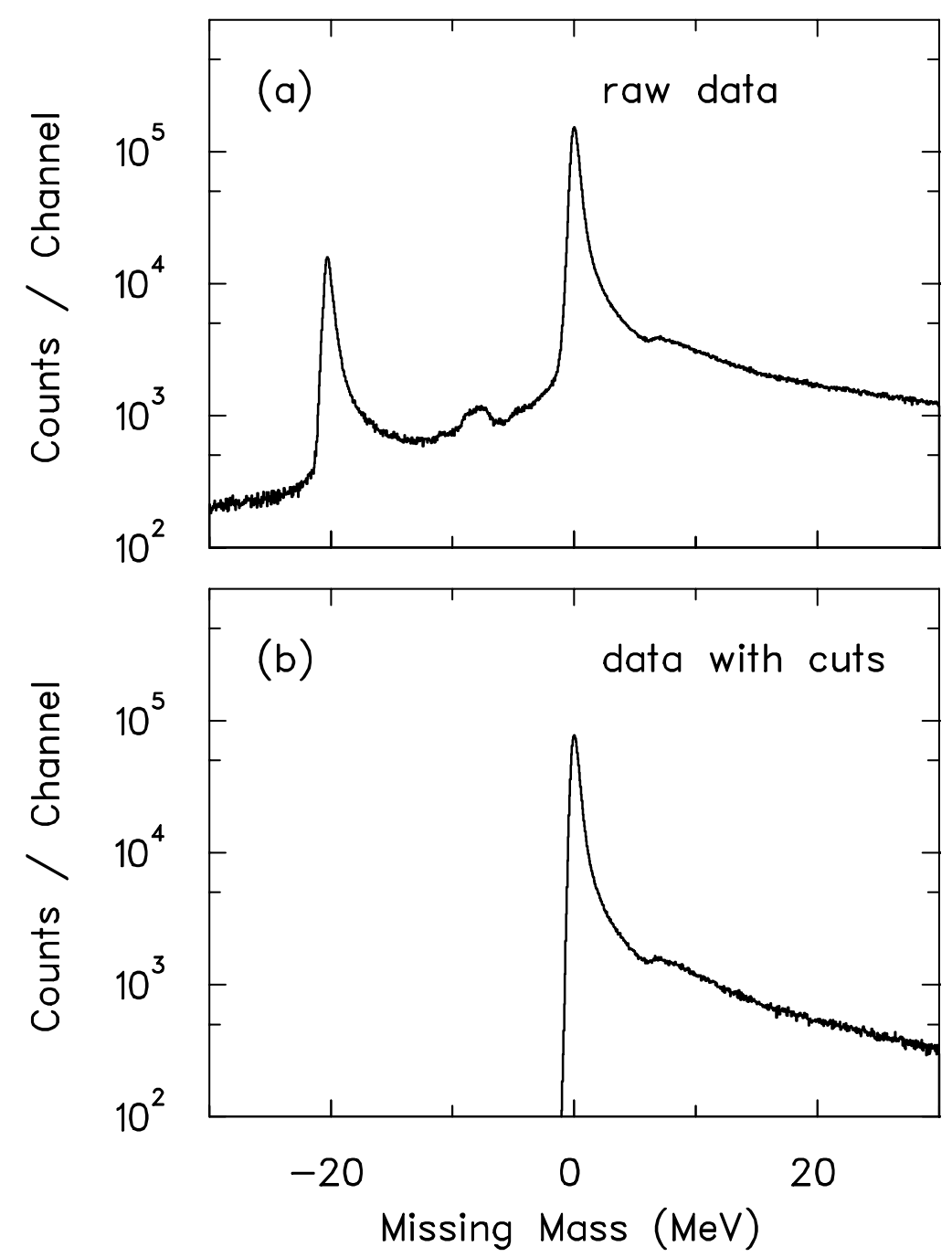

Figure 4.7: Missing mass distributions before and after the application of cuts. Plot (a) shows the missing mass distribution before any cuts were applied. At $m_{\text {miss }}=-20 \mathrm{MeV}$ the hydrogen from the snow on the target walls appears as background. The peak at $m_{\text {miss }}=6 \mathrm{MeV}$ corresponds to the two-body breakup. Plot (b) shows the same data set after making software cuts, excluding the cuts on missing mass and cuts concerning the FPP. 


\section{Chapter 5}

\section{Extraction of Polarizations}

After completion of the calibrations and getting the information about the kinematic settings, models for the spin transport in the spectrometers were used to obtain the spin transport matrices. A spin transport code to calculate the spin variables at the target using a maximum-likelihood analysis was developed. Finally, a Monte Carlo simulation was used for acceptance averaging of the theoretical calculations.

\subsection{Introduction to Polarimetry}

Proton polarimeters take advantage of the asymmetry in the scattering of polarized protons off spin-zero nuclei due to the spin-orbit $(\vec{L} \cdot \vec{S})$ dependent part of the nuclear force. For a vertically oriented spin direction, this gives rise to an asymmetry in the number of particles scattered to the left, $N_{L}$, and to the right, $N_{R}$. This left-right asymmetry $\epsilon$ is proportional to the polarization of the proton $P$ and to the carbon analyzing power $A_{C}$.

$$
\epsilon=\frac{N_{L}-N_{R}}{N_{L}+N_{R}}=A_{C} P
$$

As shown in Fig. 5.1 the polarization components of the proton transverse to the proton's momentum $\left(P_{x}\right.$ and $\left.P_{y}\right)$ result in a specific angular distribution [74]

$$
\sigma(\vartheta, \varphi)=\sigma_{0}(\vartheta) \cdot\left[1+A_{C}\left(\vartheta, T_{\mathrm{CC}}\right)\left(P_{y} \cos \varphi-P_{x} \sin \varphi\right)\right]
$$

of the proton scattering cross section $\sigma$ from the carbon nucleus with polar angle $\vartheta$ and azimuthal angle $\varphi$. In the orientation of Fig. 5.1 the $P_{x}$ polarization component leads to an up-down asymmetry and $P_{y}$ to a left-right asymmetry. False or instrumental asymmetries are neglected in Eq. 5.2 and in the following. The carbon analyzing power depends on the polar angle $\vartheta$ and the kinetic energy of the proton $T_{\mathrm{CC}}$. An example 


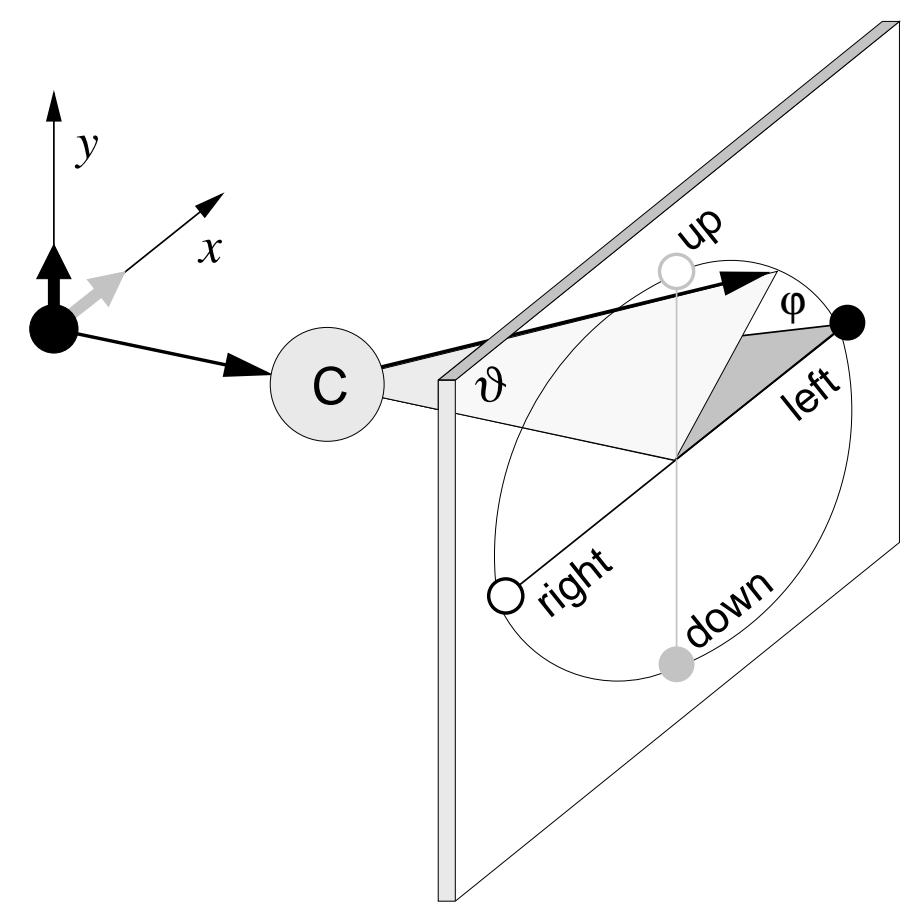

Figure 5.1: Schematic polarimeter with a polarized proton scattering from a carbon nucleus leading to an angular distribution which reveals the transverse polarization components of the proton.

of such an angular distribution from E93-049 data is shown in Fig. 5.2. The data were integrated over a $\vartheta$ range from $10^{\circ}$ to $26^{\circ}$. The statistical precision $\Delta P$ of the extracted polarization components depends on the number of incident protons $N_{0}$ and the figure of merit $F,[75]$

$$
\Delta P=\sqrt{\frac{2}{N_{0} F^{2}}} .
$$

The figure of merit characterizes the polarimeter performance in the scattering angle domain $\left(\vartheta_{\min }, \vartheta_{\max }\right)$ and is defined as

$$
F^{2}=\int_{\vartheta_{\min }}^{\vartheta_{\max }} \eta(\vartheta) A_{C}^{2}(\vartheta) d \vartheta
$$

where the polarimeter efficiency is the fraction of scatterers $\eta(\vartheta)=N(\vartheta) / N_{0}$ with polar angles $\vartheta$. Small angle scattering with $\vartheta$ of the order of $5^{\circ}$ or less is dominated by multiple Coulomb scattering and this reaction has no analyzing power. The figure of merit was maximized by choosing different analyzer thicknesses for different $Q^{2}$. An increase in carbon thickness increases the probability of useful $p C$ interactions. At the same time, however, there is also an increase in absorptive interactions which can 
reduce the efficiency and an increase in multiple Coulomb scattering which reduces the average analyzing power at small angles and the tracking resolution. An increased analyzer thickness also reduces the average kinetic energy of the protons. Since the carbon analyzing power peaks at about $T_{\mathrm{CC}}=200 \mathrm{MeV}$ this energy reduction increases the average analyzing power for energies larger than $200 \mathrm{MeV}$.

Examples of parameterizations of carbon analyzing powers and figure of merits in different regimes of kinetic energy and carbon thicknesses are those given in Ref. [74-76]. Fig. 5.3 shows the analyzing power for the Hall A FPP as measured in ${ }^{1} \mathrm{H}\left(\vec{e}, e^{\prime} \vec{p}\right)$ for three different $Q^{2}$. The experimental data are compared to the appropriate parametrizations for the respective analyzer thicknesses and proton momenta.

\subsection{Spin Transport Matrices}

For calculating the polarization components in the electron scattering plane from the polarizations as measured in the focal plane, the rotation of the spins around the magnetic field lines along the particles' flight path through the spectrometer magnets must be known. To first order, a magnetic spectrometer can be thought of as a pure dipole. In this case, the spin transport matrix can be simply written as

$$
\mathcal{S}=\left(\begin{array}{ccc}
\cos \chi & 0 & -\sin \chi \\
0 & 1 & 0 \\
\sin \chi & 0 & \cos \chi
\end{array}\right),
$$

where $\chi$ describes the change in relative angle of the spin with respect to the direction of the particle's momentum. A schematic view of the spin precession in the magnetic field of a dipole is shown in Fig. 5.4. To improve optical properties and increase their acceptance, magnetic spectrometers usually have one or several quadrupole or sextupole components. In addition, fringe fields are not negligible. Therefore, higher order corrections to the spin transport matrix as given in Eq. 5.5 have to be determined. There is no method available to easily measure the spin matrix experimentally. Hence the method of choice is to obtain an extensive magnetic field mapping for the spectrometer 
using NMR probes and studying the particle transport optics with sieve slit measure-

ments. Combining those methods leads to an optical model which is the basis for a theoretical modelling of the spin transport. There are currently two codes available to achieve this, SNAKE [77] and COSY [78].

COSY is a spin transport code which used the differential algebra technique. It allows the study and design of magneto-optic systems with arbitrary numbers of beam physics systems to arbitrary order, only limited by computing power. COSY has its own programming language, which is based on a precompiler using FORTRAN subroutines. The user interface has inherently simple but powerful syntax. The exit and entrance shapes of the dipole are known up to 5th order, therefore the matrix was calculated to the same order, using the central proton spectrometer momenta as listed in Table 4.3.

In Mainz, a different approach to calculate the spin transport matrix was chosen. The field mapping of the rather complicated magnetic field of spectrometer A was not precise enough to calculate the properties of the magneto-optic system to sufficient accuracy. Therefore, the raytracing was done experimentally, using sieve slit calibration measurements over a wide range of energies. Because the field maps were now already available in RAYTRACE-format, it was decided [48] to write a spin transport code (QSPIN) based on this format instead of converting to input formats required by COSY or SNAKE. The calculation of the magnetic fields from the input parameters is done similarly to the RAYTRACE calculations. The programming language of QSPIN is $\mathrm{C}++$, and the magnetic elements available in the code are limited to dipoles and multipoles necessary to describe spectrometer A. Other differences to RAYTRACE are the variable stepsize in the raytracing calculation and the use of other coordinate systems. Documentation for QSPIN can be found in Appendix C of [48].

\subsection{Maximum Likelihood Method}

The induced and transferred polarization components are the quantities to be determined. This can be done by means of the maximum likelihood technique (see e.g. Ref. [79]). The likelihood function $L$ is defined as a product of the probability densities 
for each of the $N$ events

$$
L=\prod_{i=1}^{N} f\left(\varphi_{i} ; \vec{P}, \vec{P}^{\prime}\right) .
$$

Only those events are included in the product where the full cone of $\varphi_{i} \in\left[0^{\circ}, 360^{\circ}\right)$ would have been observed with the same probability (cone test). As mentioned earlier we also neglect possible instrumental asymmetries. The probability density, as expressed by the asymmetries $\epsilon_{x}$ and $\epsilon_{y}$, is then given by

$$
f(\varphi)=\frac{1}{2 \pi}\left(1+\epsilon_{x} \sin \varphi+\epsilon_{y} \cos \varphi\right)
$$

The asymmetries are linear functions of, in the most general case, the induced and transferred polarization components.

$$
\begin{aligned}
\epsilon_{x} & =-A_{C} \cdot\left[S_{x x}\left(P_{x}+h P_{x}^{\prime}\right)+S_{x y}\left(P_{y}+h P_{y}^{\prime}\right)+S_{x z}\left(P_{z}+h P_{z}^{\prime}\right)\right] \\
\epsilon_{y} & =A_{C} \cdot\left[S_{y x}\left(P_{x}+h P_{x}^{\prime}\right)+S_{y y}\left(P_{y}+h P_{y}^{\prime}\right)+S_{y z}\left(P_{z}+h P_{z}^{\prime}\right)\right]
\end{aligned}
$$

The analyzing power $A_{C}$ and the spin transport coefficients $S_{i j}$ which take into account the proton spin precession in the spectrometer's magnetic fields are known or calculated on a event-by-event basis. The electron beam polarization $h$ is either positive or negative depending on the helicity state of the beam at the time the particular event was recorded. The likelihood function can be rewritten as

$$
L=\prod_{i=1}^{N} \frac{1}{2 \pi}\left(1+\sum_{k} \lambda_{k, i} P_{k}+\sum_{k} \lambda_{k, i}^{\prime} P_{k}^{\prime}\right)
$$

where $k=x, y, z$ and the coefficients $\lambda$ are given for each event individually by

$$
\begin{gathered}
\lambda_{x}=A_{C}\left(S_{y x} \cos \varphi-S_{x x} \sin \varphi\right), \\
\lambda_{y}=A_{C}\left(S_{y y} \cos \varphi-S_{x y} \sin \varphi\right), \\
\lambda_{z}=A_{C}\left(S_{y z} \cos \varphi-S_{x z} \sin \varphi\right), \\
\lambda_{x, y, z}^{\prime}=h \lambda_{x, y, z} .
\end{gathered}
$$

The maximum likelihood estimations $\hat{P}_{k}$ and $\hat{P}_{k}^{\prime}$ are those which maximize the likelihood function $L$ and are given by the solutions of the coupled nonlinear equations $\partial \ln L / \partial P_{k}=0$ and $\partial \ln L / \partial P_{k}^{\prime}=0$. These solutions are not easily obtained. A linearization of these equations simplifies the problem to the linear equation system 


$$
\underbrace{\left(\begin{array}{c}
\sum \lambda_{x} \\
\sum \lambda_{y} \\
\vdots \\
\sum \lambda_{z}^{\prime}
\end{array}\right)}_{\mathrm{B}}=\underbrace{\left(\begin{array}{cccc}
\sum \lambda_{x} \lambda_{x} & \sum \lambda_{x} \lambda_{y} & \cdots & \sum \lambda_{x} \lambda_{z}^{\prime} \\
\sum \lambda_{y} \lambda_{x} & \sum \lambda_{y} \lambda_{y} & \cdots & \sum \lambda_{y} \lambda_{z}^{\prime} \\
\vdots & \vdots & \ddots & \vdots \\
\sum \lambda_{z}^{\prime} \lambda_{x} & \sum \lambda_{z}^{\prime} \lambda_{y} & \cdots & \sum \lambda_{z}^{\prime} \lambda_{z}^{\prime}
\end{array}\right)}_{\mathrm{F}}\left(\begin{array}{c}
\hat{P}_{x} \\
\hat{P}_{y} \\
\vdots \\
\hat{P}_{z}^{\prime}
\end{array}\right) .
$$

The sums are carried out over all events. The polarization estimates are thus given by

$$
\left(\hat{P}_{x}, \hat{P}_{y}, \ldots, \hat{P}_{z}^{\prime}\right)^{\mathrm{T}}=\mathrm{F}^{-1} \mathrm{~B}
$$

with covariance matrix $\mathrm{F}^{-1}$.

\subsection{FPP Event Selection}

The last set of event selections concerned the secondary scattered events in the FPP. As can be seen in Fig. 4.4, some protons are scattered off e.g. the rear FPP chamber which are at $z_{\text {close }}=395 \mathrm{~cm}$. Therefore, events for which the incident and scattered tracks do not intersect within the physical limits of the carbon were removed. Table 5.1 lists the $z_{\text {close }}$ cuts applied; note that the origin of the coordinate system was defined differently for Mainz. Scattering events for $\vartheta<3^{\circ}$ are dominated by Coulomb scattering with

\begin{tabular}{lrc}
\hline \hline $\begin{array}{l}Q^{2} \\
\left(\mathrm{GeV}^{2}\right)\end{array}$ & $\begin{array}{c}z_{\text {close }}^{\text {min }} \\
(\mathrm{m})\end{array}$ & $\begin{array}{c}z_{\text {close }}^{\text {max }} \\
(\mathrm{m})\end{array}$ \\
\hline 0.4 & -200 & -40 \\
0.5 & 367 & 389 \\
1.0 & 323 & 389 \\
1.6 & 323 & 383 \\
2.6 & 323 & 383 \\
\hline \hline
\end{tabular}

Table 5.1: Software cuts on $z_{\text {close }}$.

no analyzing power and were not analyzed. Events at angles larger than $70^{\circ}$, where the analyzing power has already dropped rapidly, were also excluded. For Mainz, these cuts were set even tighter to $7^{\circ}<\vartheta<35^{\circ}$. To avoid introducing false asymmetries, a 
cone test was applied. The test is passed by an event if all tracks with the same angle $\vartheta$ around the incident track are within the acceptance of the rear FPP chamber.

\subsection{Systematic Uncertainties}

There are three sources of systematic uncertainties in the experiment. Uncertainty on the target coordinate reconstruction due to the knowledge of spectrometer optics, uncertainty in the spin transport and false asymmetries in the spectrometers. Estimates of the systematic uncertainties were obtained by examining the sensitivity of the results on input parameters.

\section{Spectrometer Optics}

The optics of the JLab Hall A Spectrometers were extensively studied in [71]. The angular and vertex resolutions at the target were estimated to be

$$
\begin{aligned}
\Delta \theta^{\mathrm{tg}} & =6.2 \mathrm{mrad} \\
\Delta \Phi^{\mathrm{tg}} & =2.0 \mathrm{mrad} \\
\Delta y^{\mathrm{tg}} & =3.6 \mathrm{~mm} .
\end{aligned}
$$

These values were used as a very conservative base for a possible systematic shift in the input parameters. Table 5.2 shows the results for the studies of the systematic uncertainties due to the spectrometer optics. Listed are the changes in

$$
R_{\mathrm{H}}=\left(\frac{P_{x}^{\prime}}{P_{x}^{\prime}}\right)_{\mathrm{H}}, \quad R_{\mathrm{He}}=\left(\frac{P_{x}^{\prime}}{P_{x}^{\prime}}\right)_{\mathrm{He}} \quad \text { and } \quad R=\left(\frac{R_{\mathrm{He}}}{R_{\mathrm{H}}}\right)
$$

relative to the nominal values for a change of the constant in the respective column. The shifts in these constants were $6 \mathrm{mrad}$ for $\Delta \theta^{\operatorname{tg}}, 2 \mathrm{mrad}$ for $\Delta \Phi^{\mathrm{tg}}, 2 \mathrm{~mm}$ for $\Delta y^{\operatorname{tg}}$ and $2 \cdot 10^{-4}$ for $\Delta p_{p}$. It can be seen that the changes in $R_{\mathrm{H}}$ and $R_{\mathrm{He}}$ are in the same direction in most cases, so their systematic uncertainties cancel largely for the super ratio $R$. This is not the case for the measurement at $Q^{2}=2.6 \mathrm{GeV}^{2}$, because there we have no hydrogen measurement. For all other cases, the changes given in the table are a comfortable upper limit. The most recent numbers for uncertainties in the spectrometer input parameters are estimated to be $\Delta \theta^{\operatorname{tg}}=\Delta \Phi^{\operatorname{tg}}=0.8 \mathrm{mrad}$ and $\Delta y^{\operatorname{tg}}=0.4 \mathrm{~mm}$. 
Therefore, systematic errors of all optical variables combined is estimated to be lower than $1 \%$ on $R$ for the three lower $Q^{2}$ points.

\begin{tabular}{llcccc}
\hline \hline $\begin{array}{l}Q^{2} \\
\left(\mathrm{GeV}^{2}\right)\end{array}$ & & $\begin{array}{c}\Delta \theta^{\mathrm{tg}} \\
\%\end{array}$ & $\begin{array}{c}\Delta \Phi^{\mathrm{tg}} \\
\%\end{array}$ & $\begin{array}{c}\Delta y^{\mathrm{tg}} \\
\%\end{array}$ & $\begin{array}{c}\Delta p_{p} \\
\%\end{array}$ \\
\hline \hline 0.5 & $R_{\mathrm{H}}$ & -0.2 & -0.9 & -0.4 & +0.3 \\
& $R_{\mathrm{He}}$ & +0.4 & -1.1 & +0.1 & +0.6 \\
& $R$ & +0.6 & -0.2 & +0.5 & +0.3 \\
\hline 1.0 & $R_{\mathrm{H}}$ & +1.5 & -0.3 & -0.0 & +1.1 \\
& $R_{\mathrm{He}}$ & -2.4 & -1.6 & -0.9 & +0.4 \\
& $R$ & -0.8 & -1.3 & -0.9 & -0.8 \\
\hline 1.6 & $R_{\mathrm{H}}$ & -4.9 & -1.0 & -2.1 & +0.2 \\
& $R_{\mathrm{He}}$ & -6.2 & -1.0 & -1.9 & +0.3 \\
& $R$ & -1.3 & -0.2 & +0.3 & +0.2 \\
\hline 2.6 & $R$ & +7.8 & -1.5 & -4.2 & +0.1 \\
\hline \hline
\end{tabular}

Table 5.2: Systematic uncertainties caused by spectrometer optics.

\section{Spin Transport}

A similar study on systematic errors was done for the $Q^{2}$ points at $1.0 \mathrm{GeV}^{2}$ and 1.6 $\mathrm{GeV}^{2}$. Spin transport matrices were calculated with one of the quadrupoles either shifted by $2 \mathrm{~mm}$ in the longitudinal (long) or transverse (tran) direction, or rotated (rot) by 2 mrad in the counterclockwise direction. Also, the shim angles of the dipole (shim) were changed by 2 degrees. Table 5.3 gives the deviations from $R$ in percent for these studies. The conclusion is that systematic errors from the spin transport are well below $1 \%$ using the current knowledge of the Hall A spectrometer optics.

\section{Analyzing Power and False Asymmetries}

Systematic uncertainties due to the knowledge of the analyzing power were studied by analyzing the data with parametrizations which do not fit the kinematic range and carbon thicknesses. Even setting the analyzing power $A_{c}=1$ led to a change in $R$ of at most $1 \%$, using other parametrizations, $0.2 \%$. Instrumental asymmetries can originate from detector misalignments or inhomogenities of detector responses. The detector response $\eta_{d}(\vartheta, \varphi)$ including these false asymmetries can be written as a Fourier 


\begin{tabular}{lccc}
\hline \hline Magnet & Shift & $1.0 \mathrm{GeV}^{2}$ & $1.6 \mathrm{GeV}^{2}$ \\
\hline \hline Q1 & long & -0.4 & -0.2 \\
Q1 & tran & -0.2 & -0.1 \\
Q1 & rot & -0.4 & -0.0 \\
\hline Q2 & long & -0.3 & -0.1 \\
Q2 & tran & -0.2 & -0.1 \\
Q2 & rot & -0.4 & -0.0 \\
\hline Q3 & long & -0.3 & -0.0 \\
Q3 & tran & -0.3 & -0.0 \\
Q3 & rot & -0.3 & -0.1 \\
\hline D & shim & -0.3 & -0.0 \\
\hline \hline
\end{tabular}

Table 5.3: Systematic uncertainties in $\mathrm{R}$ caused by uncertainties in the spin transport.

expansion:

$$
\eta_{d}(\vartheta, \varphi)=\eta_{0}(\vartheta)\left[1+\sum a_{i} \sin (i \varphi)+\sum b_{i} \cos (i \varphi)\right]
$$

The experimentally observed asymmetries $(\bar{\epsilon})_{t}$ and $\bar{\epsilon}_{n}$ are given by

$$
\begin{aligned}
& \bar{\epsilon}_{t}=\epsilon_{t}+a_{i}+\frac{1}{2}\left(\epsilon_{n} a_{2}-\epsilon_{t} b_{2}\right) \\
& \bar{\epsilon}_{t}=\epsilon_{n}+b_{i}+\frac{1}{2}\left(\epsilon_{n} b_{2}+\epsilon_{t} a_{2}\right),
\end{aligned}
$$

with $\epsilon_{t}$ and $\epsilon_{n}$ as the physics asymmetries. Reduction of the false asymmetries is achieved by several means. First, a cone test is applied, in which only events lying in the full cone of $\varphi \in\left[0^{\circ}, 360^{\circ}\right)$ are accepted. As can be easily seen from Eqs. 5.22 and 5.23 , flipping the sign of physics asymmetries does not affect the contibution to the first order instrumental asymmetries. Thus, first order false asymmetries largely cancel for the polarization transfer ratio. Second order false asymmetries, which appear in the bilinear terms of Eqs. 5.22, are very small and do not contribute significantly to systematic errors for the polarization transfer ratio. 


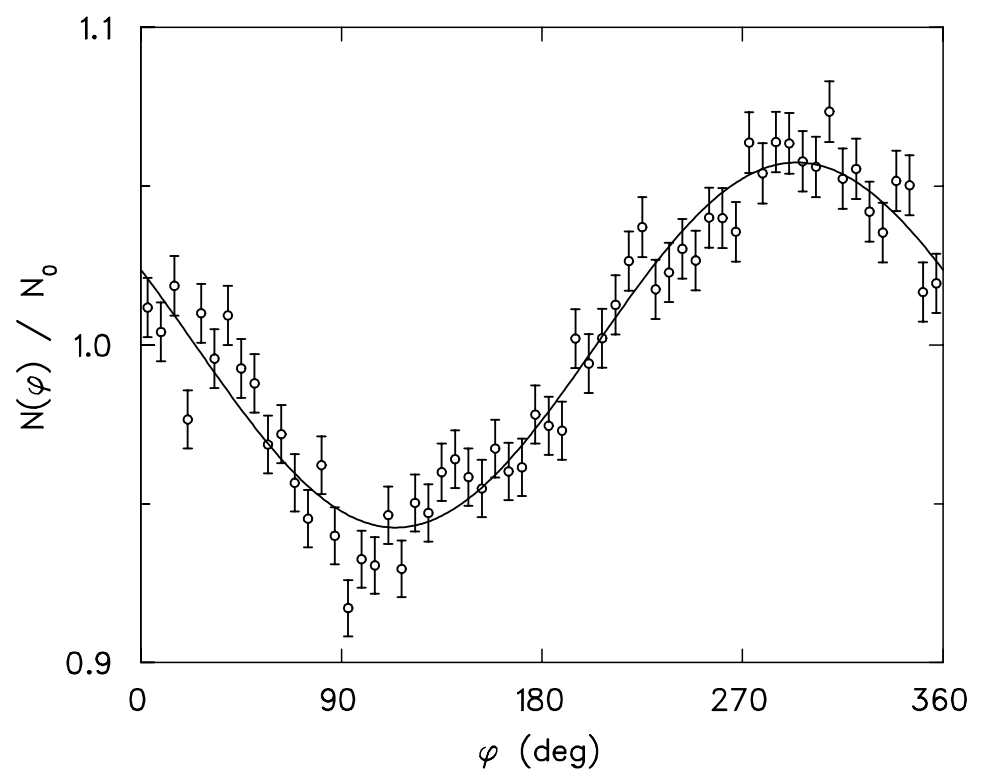

Figure 5.2: Example of an angular distribution of polarized protons scattered from a carbon analyzer. Data are taken from ${ }^{1} \mathrm{H}\left(\vec{e}, e^{\prime} \vec{p}\right)$. The solid line is a fit of Eq. 5.2 to the data.

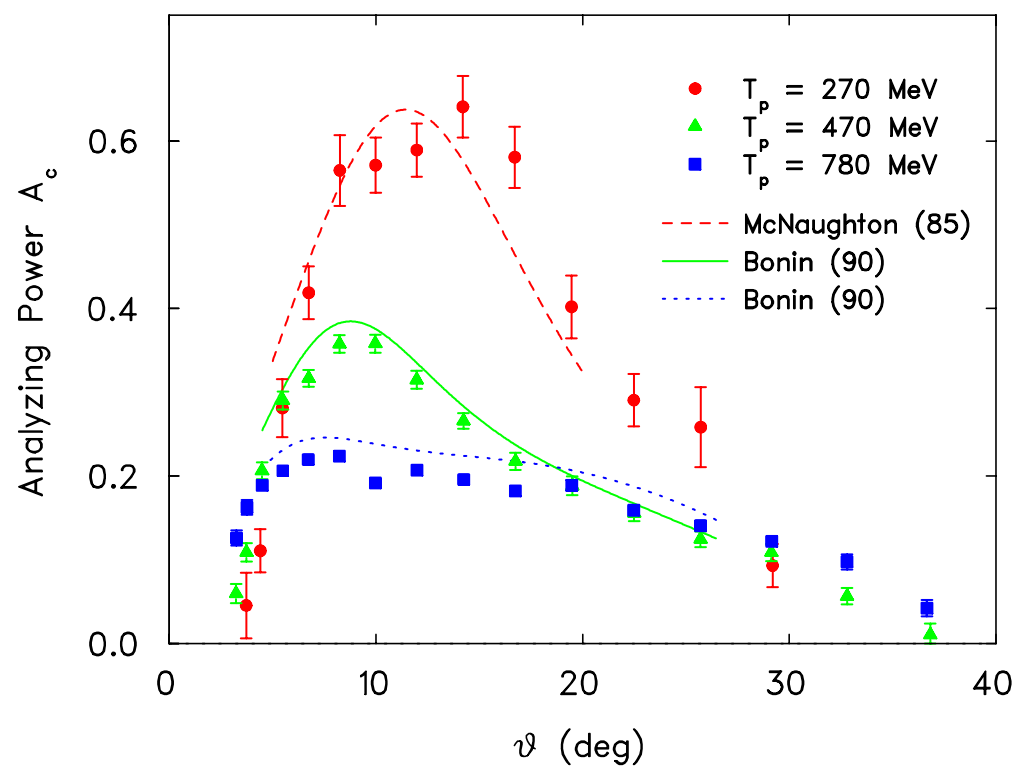

Figure 5.3: Analyzing power of the Hall A FPP as measured in ${ }^{1} \mathrm{H}\left(\vec{e}, e^{\prime} \vec{p}\right)$ during E93-049. 


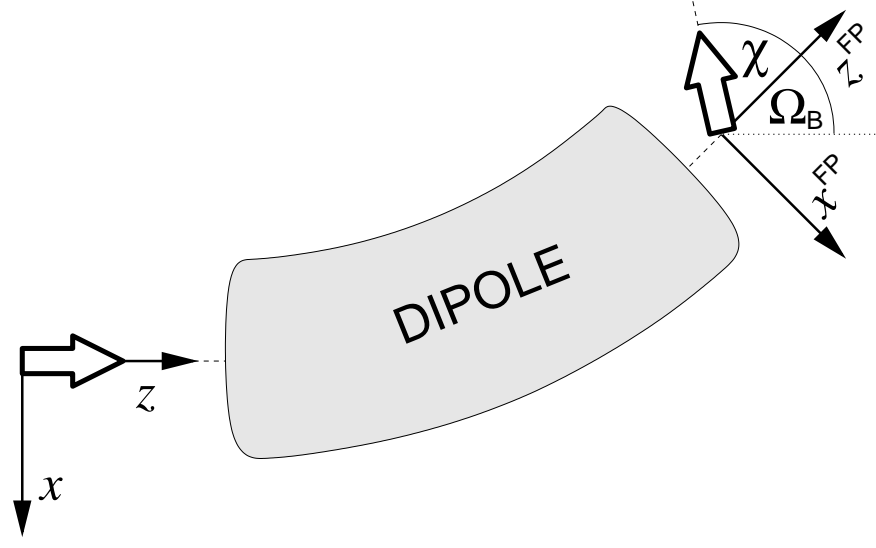

Figure 5.4: Schematic view of the spin precession in the magnetic field of a dipole. The proton spin is indicated by an open arrow. The precession angle $\chi$ is the difference between the spin and momentum rotation. The spectrometer bending angle is denoted as $\Omega_{B}$. 


\section{Chapter 6}

\section{Results and Discussion}

\subsection{Introduction}

The discussion of the results involves three main steps. The results for $G_{E}^{p} / G_{M}^{p}$ will be discussed with respect to previous experiments and compared to theoretical predictions from various models. Before the ${ }^{4} \mathrm{He}\left(\vec{e}, e^{\prime} \vec{p}\right)^{3} \mathrm{H}$ data can be interpreted, phase space averaging of the theoretical models using a Monte Carlo code was necessary. In all figures the statistical uncertainties are shown. Systematic uncertainties are small; their source was discussed in Chap. 5.

\subsection{Hydrogen Results}

The results of the analysis presented in the last chapter have to be interpreted within the context of previous experiments and available theoretical predictions. Table 6.1 shows the results for the ratio $G_{E}^{p} / G_{M}^{p}$ measured at Mainz and JLab. Figure 6.1 shows

\begin{tabular}{l|cccc}
\hline \hline$Q^{2}(\mathrm{GeV})^{2}$ & 0.4 & 0.5 & 1.0 & 1.6 \\
\hline$\mu G_{E} / G_{M}$ & $1.022 \pm 0.012$ & $0.955 \pm 0.027$ & $0.893 \pm 0.018$ & $0.790 \pm 0.017$ \\
\hline \hline
\end{tabular}

Table 6.1: Form factor ratio $\mu G_{E} / G_{M}$ for hydrogen.

the results of this experiment compared to results of previous experiments [3] in the same $Q^{2}$ range using the polarization ratio technique. There is excellent agreement with previous data and strong support for the $G_{E}^{p} / G_{M}^{p}$ ratio decreasing with $Q^{2}$.

Based on the data of [11], several theoretical studies have been made, focussing on different effects within the framework of the constituent quark model. Figure 6.2 shows the data from this work and [11] in comparison to four theoretical calculations. 


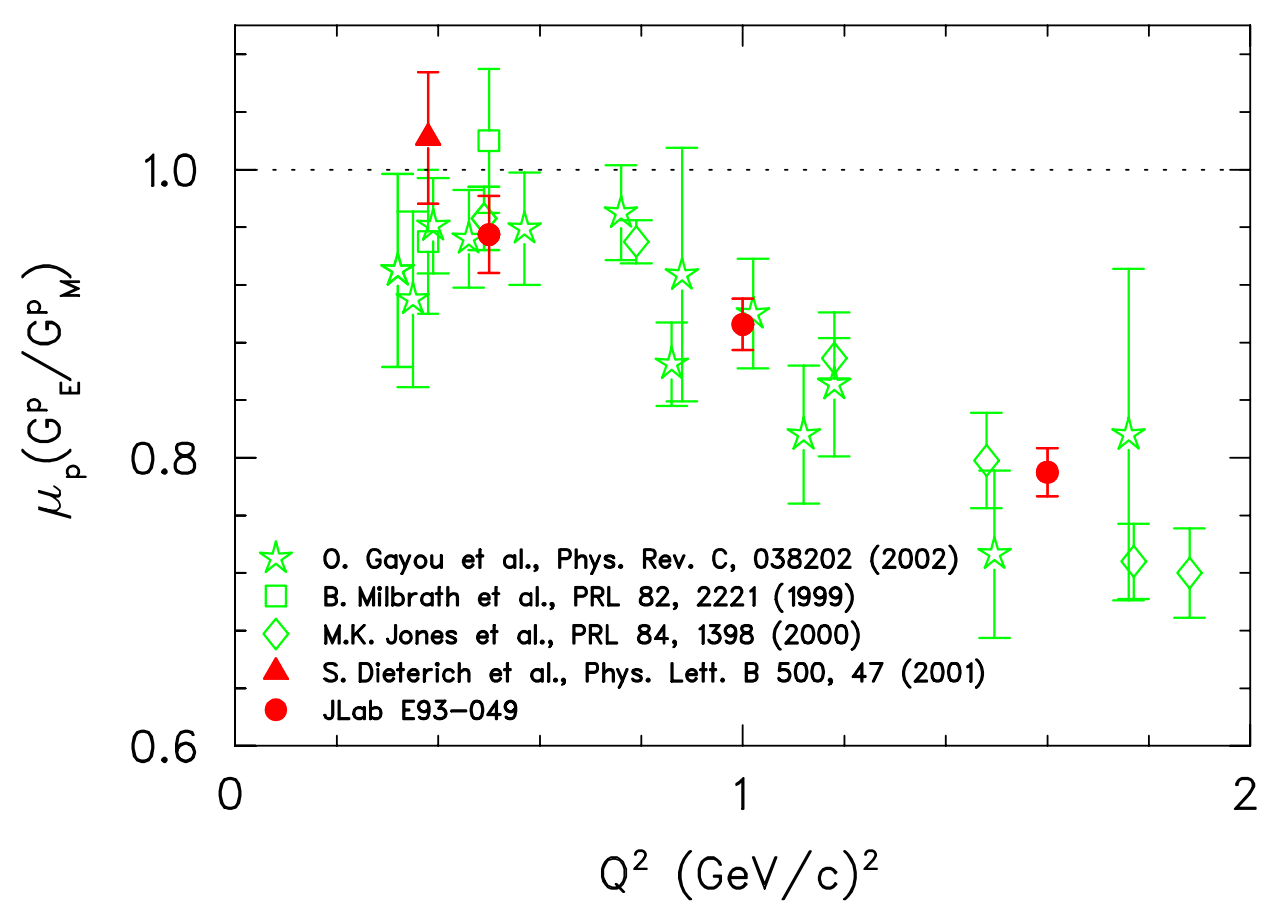

Figure 6.1: Experimental results for $\mu G_{E} / G_{M}$ in hydrogen elastic scattering. Solid circles are data from this thesis, other symbols from previous polarization transfer ratio measurements at JLab and MIT-Bates.

The Cloudy Bag Model [80], Vector Meson Dominance Model [81] and the diquark model [82] all fail to describe the data. Most notable is that each of the three models predict a leveling off of $G_{E}^{p} / G_{M}^{p}$. The Constituent Quark Model [83] agrees remarkably well with the data.

\subsection{Acceptance averaging}

Due to the large spectrometer acceptances the ${ }^{4} \mathrm{He}\left(\vec{e}, e^{\prime} \vec{p}\right)^{3} \mathrm{H}$ reaction covers a range of $Q^{2}$ around the central value, while theoretical models calculate only a point acceptance. To account for the spectrometer acceptances necessitates an accurate folding of the theoretical models calculating point acceptances to compare with data.

For each of the theories a two-dimensional grid in the variables $\omega$ and $|q|$ was constructed. Table 6.2 shows the extent of the grids and the step sizes for each $Q^{2}$ point. For each of the grid points the theoretical values of $P_{x}^{\prime}$ and $P_{z}^{\prime}$ were calculated. For the phase space averaging between the grid points a linear interpolation was used.

The Monte Carlo code MCEEP (Monte Carlo for $\left(e, e^{\prime} p\right)$ ) [84] was used to perform 


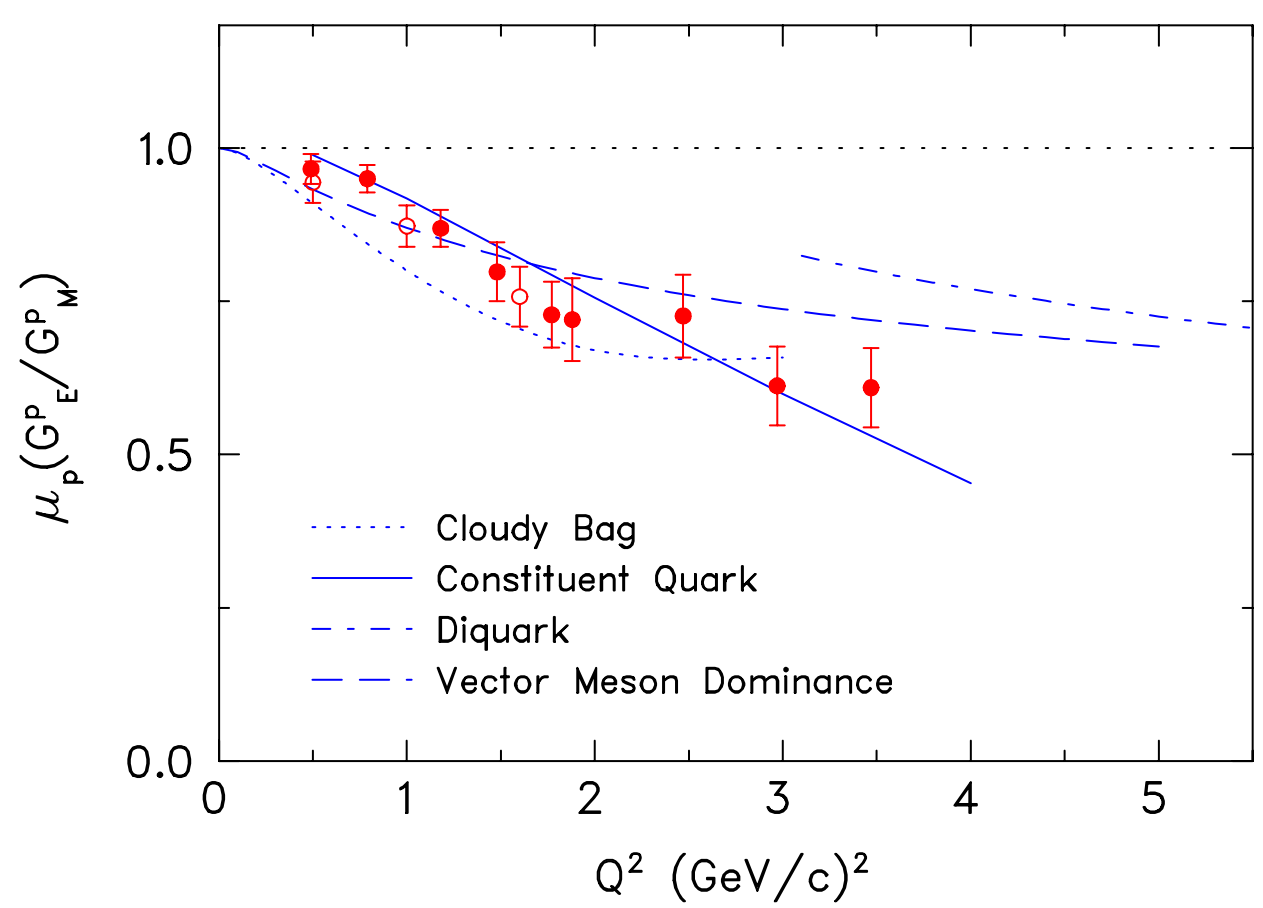

Figure 6.2: Ratio $\mu G_{E} / G_{M}$ from experimental data compared with theoretical calculations. The open circles represent the hydrogen data taken during E93-049, closed circles are data from [11].

\begin{tabular}{r|rrcrcc}
\hline \hline$Q^{2}$ & $\omega_{\min }$ & $\omega_{\max }$ & $\omega_{\text {step }}$ & $|q|_{\min }$ & $|q|_{\max }$ & $|q|_{\text {step }}$ \\
\hline 0.5 & 270 & 330 & 10 & 620 & 880 & 20 \\
1.0 & 510 & 620 & 10 & 980 & 1305 & 25 \\
1.6 & 815 & 980 & 15 & 1360 & 1685 & 25 \\
2.6 & 1134 & 1540 & 15 & 1970 & 2290 & 40 \\
\hline \hline
\end{tabular}

Table 6.2: Limits on the grid variables $\omega$ and $|q|$ for phase space averaging. The unit of $Q^{2}$ is $(\mathrm{GeV} / \mathrm{c})^{2}$, all other variables are given in $\mathrm{MeV}$.

the acceptance averaging. The agreement between the simulation and data is very good; small differences which would result in a big systematic error in cross section measurements largely cancel in the polarization transfer ratio. Errors in the phase space averaging of the theories due to differences between simulation and experiment are very small. 


\subsection{Helium Results}

As discussed in Sec. 2.1.2, Eq. 2.22 does not hold exactly for nucleon knockout reactions of the type $A\left(\vec{e}, e^{\prime} \vec{p}\right) B$. Therefore the ${ }^{4} \mathrm{He}\left(\vec{e}, e^{\prime} \vec{p}\right)^{3} \mathrm{H}$ results will be given as a ratio of the transferred polarizations:

$$
R=\frac{P_{x}^{\prime}}{P_{z}^{\prime}}
$$

The experimental super-ratio $R^{\mathrm{Exp}}$ and the PWIA supperratio $R^{\mathrm{PWIA}}$ are defined as

$$
R^{\mathrm{Exp}}=\frac{R_{\mathrm{He}}^{\mathrm{Exp}}}{R_{\mathrm{H}}^{\mathrm{Exp}}} \quad \text { and } \quad R^{\mathrm{PWIA}}=\frac{R_{\mathrm{He}}^{\mathrm{PWIA}}}{R_{\mathrm{H}}^{\mathrm{PWIA}}} .
$$

Figure 6.3 shows the range of super-ratio $R^{\exp }$ for different current operator. Three different $Q^{2}$ are shown as a function of the missing momentum. The black circles indicate the data in four bins, their numerical values are listed in Table 6.3. The

\begin{tabular}{l|rcc}
\hline \hline$Q^{2}(\mathrm{GeV})^{2}$ & $p_{m}(\mathrm{MeV})$ & $\mathrm{R}$ & $\Delta \mathrm{R}$ \\
\hline 0.5 & -151 & 0.817 & 0.087 \\
& -69 & 0.926 & 0.055 \\
& 69 & 0.884 & 0.056 \\
& 151 & 0.722 & 0.107 \\
\hline 1.0 & -151 & 0.766 & 0.070 \\
& -69 & 0.867 & 0.045 \\
& 69 & 0.908 & 0.044 \\
& 151 & 0.877 & 0.084 \\
\hline 1.6 & -151 & 0.810 & 0.063 \\
& -69 & 0.921 & 0.045 \\
& 69 & 1.014 & 0.048 \\
& 151 & 1.050 & 0.089 \\
\hline \hline
\end{tabular}

Table 6.3: Super-ratio as function of missing momentum.

upper band indicates PWIA calculations, the middle band RDWIA calculations, and the lower band RDWIA+QMC calculations. All calculations are from the Madrid code and averaged over the acceptance. The respective calculations are using different current operators. Namely, the calculation with the higher $R^{\exp }$ was done with the $c c 2$ current operator, the other with the $c c 1$ current operator [20].

In Fig. 6.4 the range of the ratio $R^{\exp }$ as a function of the missing momentum for four different wave functions [85] and three different $Q^{2}$ using the $c c 1$ current operator is 


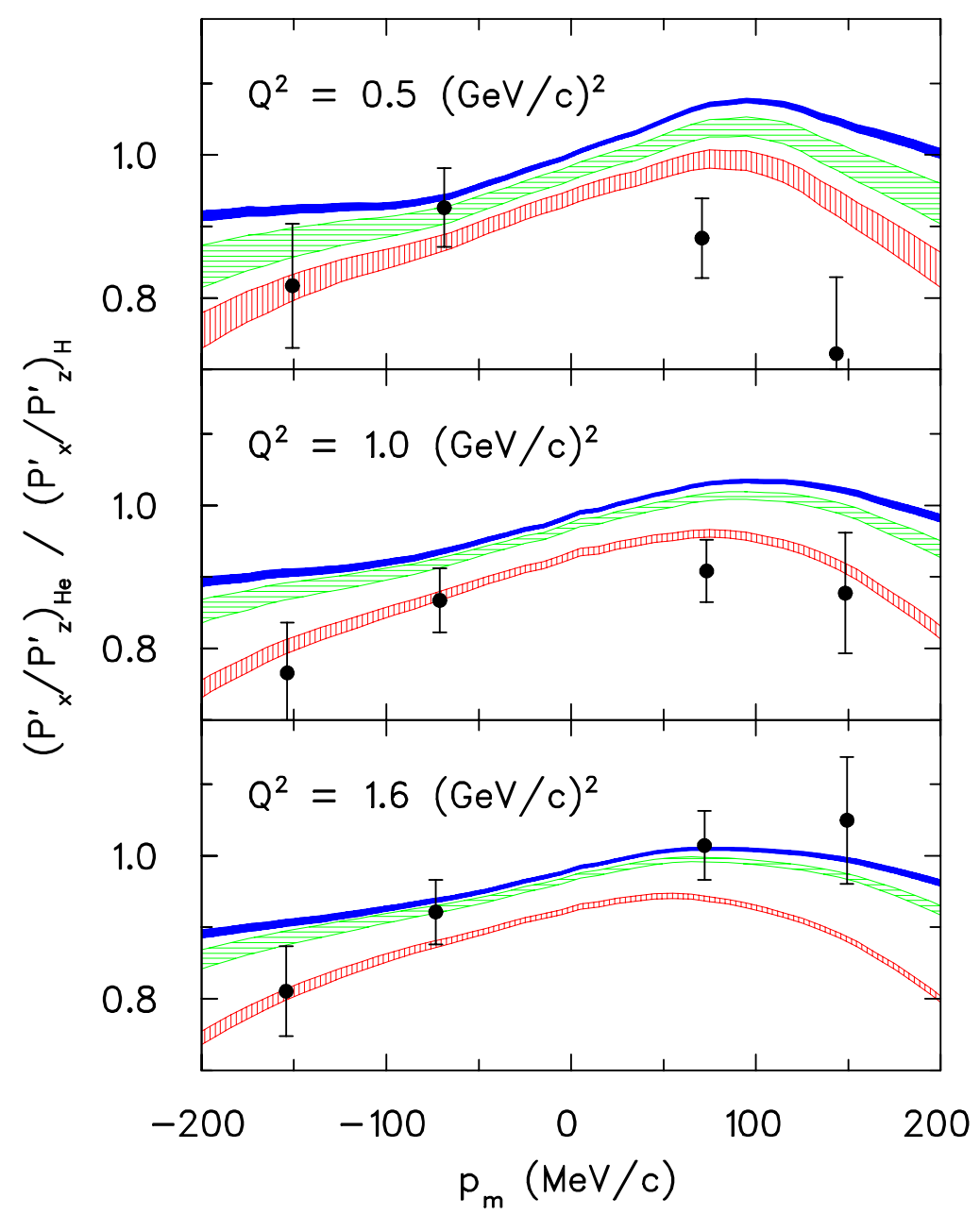

Figure 6.3: Dependency of $R^{\exp }$ on current operator. The shaded areas indicate the range of $R^{\exp }$ for different current operators $(c c 1, c c 2)$ used in the theoretical calculation. 
shown as shaded bands. The experimental data is identical to the data shown in Fig. 6.3 and listed in Table 6.3. Two come from relativistic mean field calculations, one intended for nuclear matter and the other fitted to properties to medium and heavy magic nuclei. The latter compares very badly with experimentally measured momentum distributions in ${ }^{4} \mathrm{He}\left(\vec{e}, e^{\prime} \vec{p}\right)^{3} \mathrm{H}$. The third wave function used is a is a solution of a Dirac equation with standard Woods-Saxon wells for the S and V potentials. The parameters of the Woods-Saxon well are fitted to the measured rms and binding energy of ${ }^{4} \mathrm{He}$. This wave function reproduces the ${ }^{4} \mathrm{He}\left(\vec{e}, e^{\prime} \vec{p}\right)^{3} \mathrm{H}$ momentum distribution well. The fourth wave function is based on a sum of Gaussians fit to electron elastic scattering on ${ }^{4} \mathrm{He}$ using the charge form factor. The theoretical super-ratio is insensitive to the choice of wave function, even though they vary widely.

While all three models fit the shape of the data distribution, PWIA is clearly not adequate to describe the data. Even the RDWIA, though it is only $1 \sigma$ away from the experimental value, is systematically too high. Using RDWIA+QMC gives the best description of the data. We can conclude that the theoretical interpretation of the data is not sensitive to the chosen wave function. For the current operator, $c c 1$ gives the lower ratio than $c c 2$ and is therefore more appropriate to describe our data.

In Fig. 6.5, the super-ratio $R$ at $Q^{2}=0.4 \mathrm{GeV}^{2}$ is shown compared to a variety of calculations. The result of the non-relativistic calculations of Laget, which include MEC and IC, is nearly identical to PWIA. This indicates that MEC and IC do not contribute significantly to $R$ in this kinematics. The PWIA calculation from Madrid includes positive and negative energy components for the bound state, but only positive energy components for the ejected nucleon. It gives nearly the same result as the PWIA by Laget, indicating that $R$ is insensitive to the negative energy components of the bound state. The PWIA calculations are independent of the current operator. The magnitude of the relativistic effects, as can be seen by comparing RDWIA and PWIA from the Madrid model, is significant. For the off shell current operators, the ratio is substantially less for CC1 than for CC2.

To compare $R$ for different $Q^{2}$, the difference which comes from the kinematics alone 


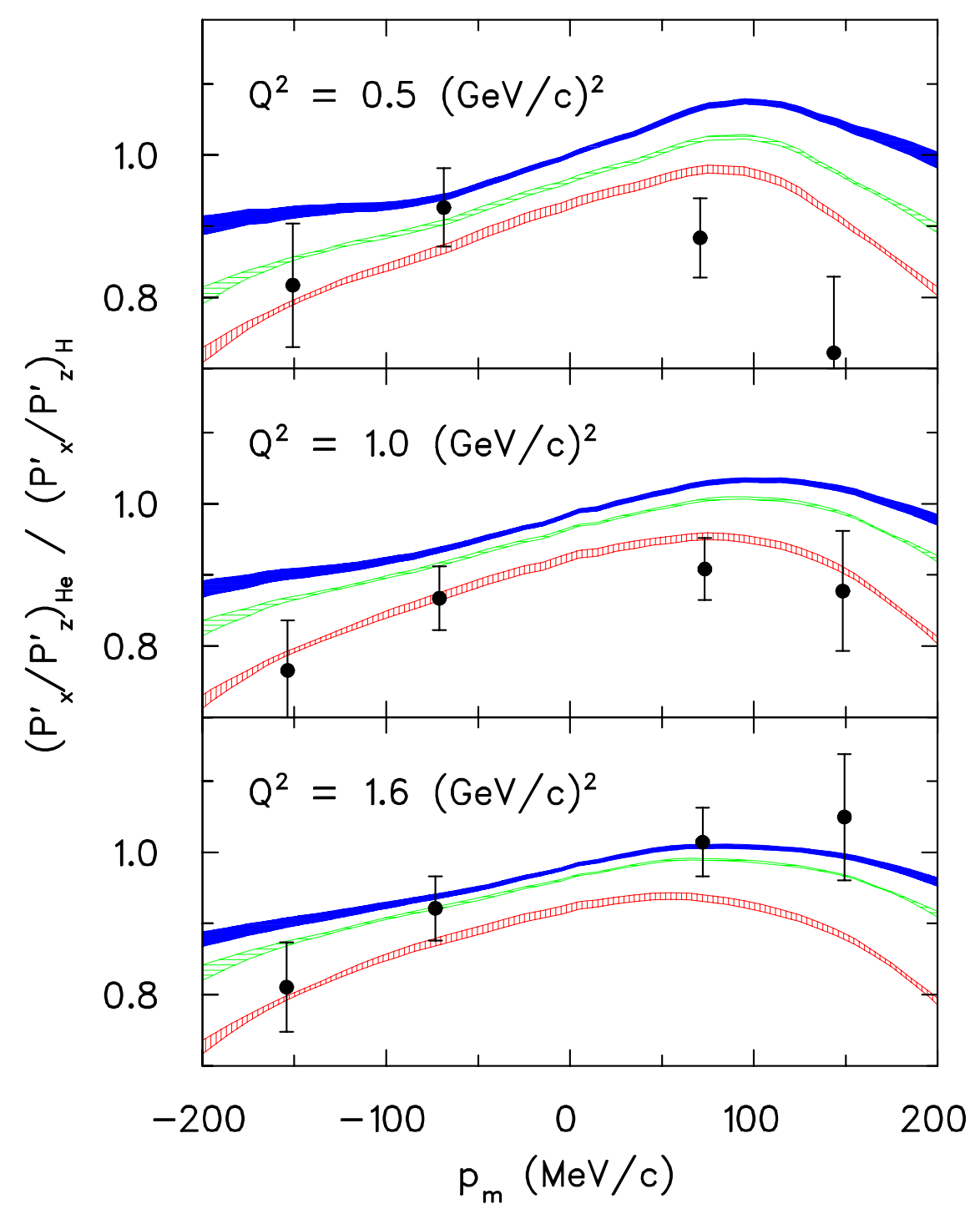

Figure 6.4: Dependency of $R^{\exp }$ on wave functions. The shaded areas indicate the range of $R^{\exp }$ for different wave functions used in the theoretical calculations. 


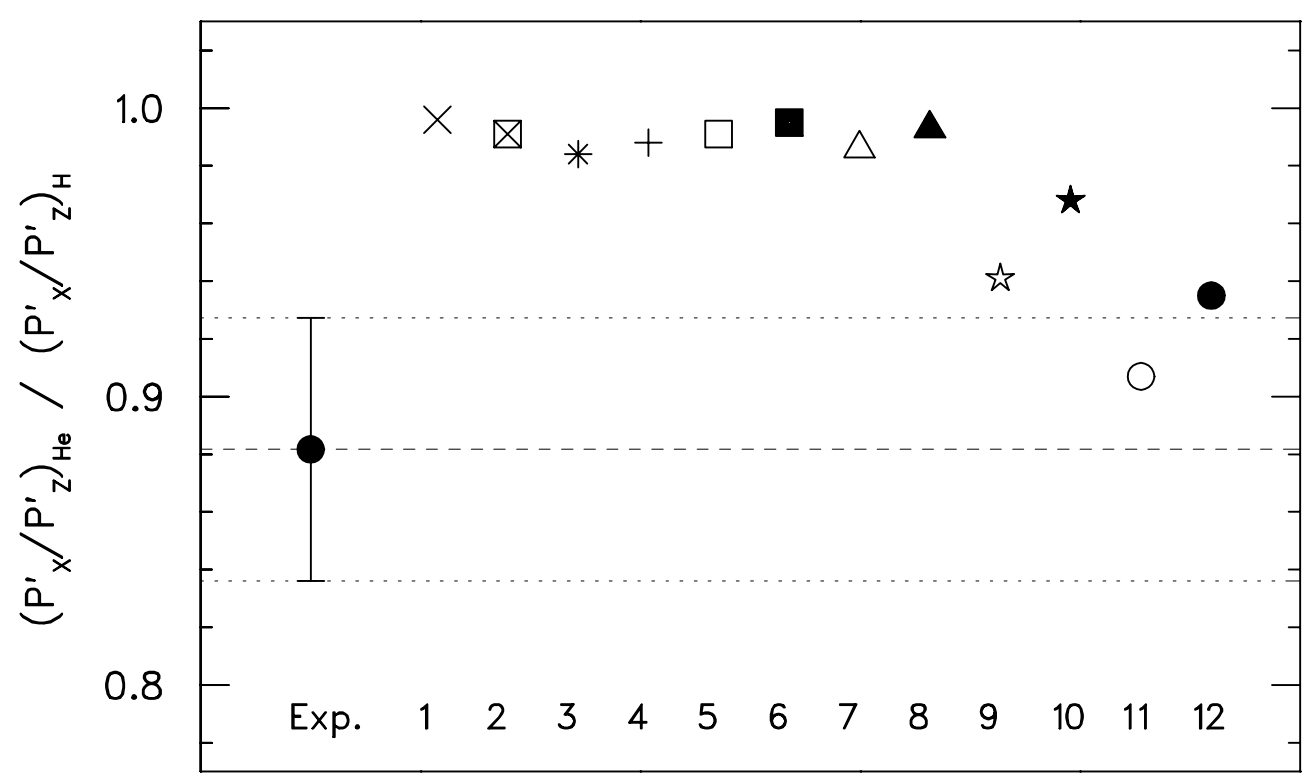

Figure 6.5: Comparison of $R^{\exp }$ measured at $Q^{2}=0.4 \mathrm{GeV}^{2}$ in Mainz with theoretical calculations . Laget - PWIA (1); full calculation (2). Madrid - PWIA, $c c 1$ (3), cc2 (4); positive energy projection, $c c 1$ (5), cc2 (6); no spinor distortions, $c c 1$ (7), cc2 (8); RDWIA, $c c 1$ (9), $c c 2$ (10); RDWIA+QMC, $c c 1$ (11), cc2 (12).

has to be accounted for. This can be done by normalizing $R$ to the PWIA value. Figure 6.6 shows $R$ for all $Q^{2}$ with the Madrid PWIA as a baseline. All experimental values are below PWIA. The data points taken at Mainz and at $Q^{2}=0.5 \mathrm{GeV}^{2}$ from JLab agree very well. Phase-space averaged theoretical results are also shown, connected to guide the eye. The RDWIA value is, as seen in previous plots, too high for almost all $Q^{2}$. Including QMC improves the description of the data considerably. The diamonds connected by the dashed-dotted line are from a calculation by Laget including two-body currents. It fails to describe the $Q^{2}$ dependence of the experimental data.

\subsection{Quark-Hadron Duality and the Nuclear EMC Effect}

Density dependent effects modifying the quark structure of the nucleon should leave traces in a variety of processes and observables. In [86] the relation between medium modifications and changes of the deep inelastic structure functions at high Bjorken $x$ was studied using the relations of quark-hadron duality. Under the assumption that local duality is a good approximation, these relations are model independent. Global scaling was observed originally by Bloom and Gilman [87], who noted that the $F_{2}$ 


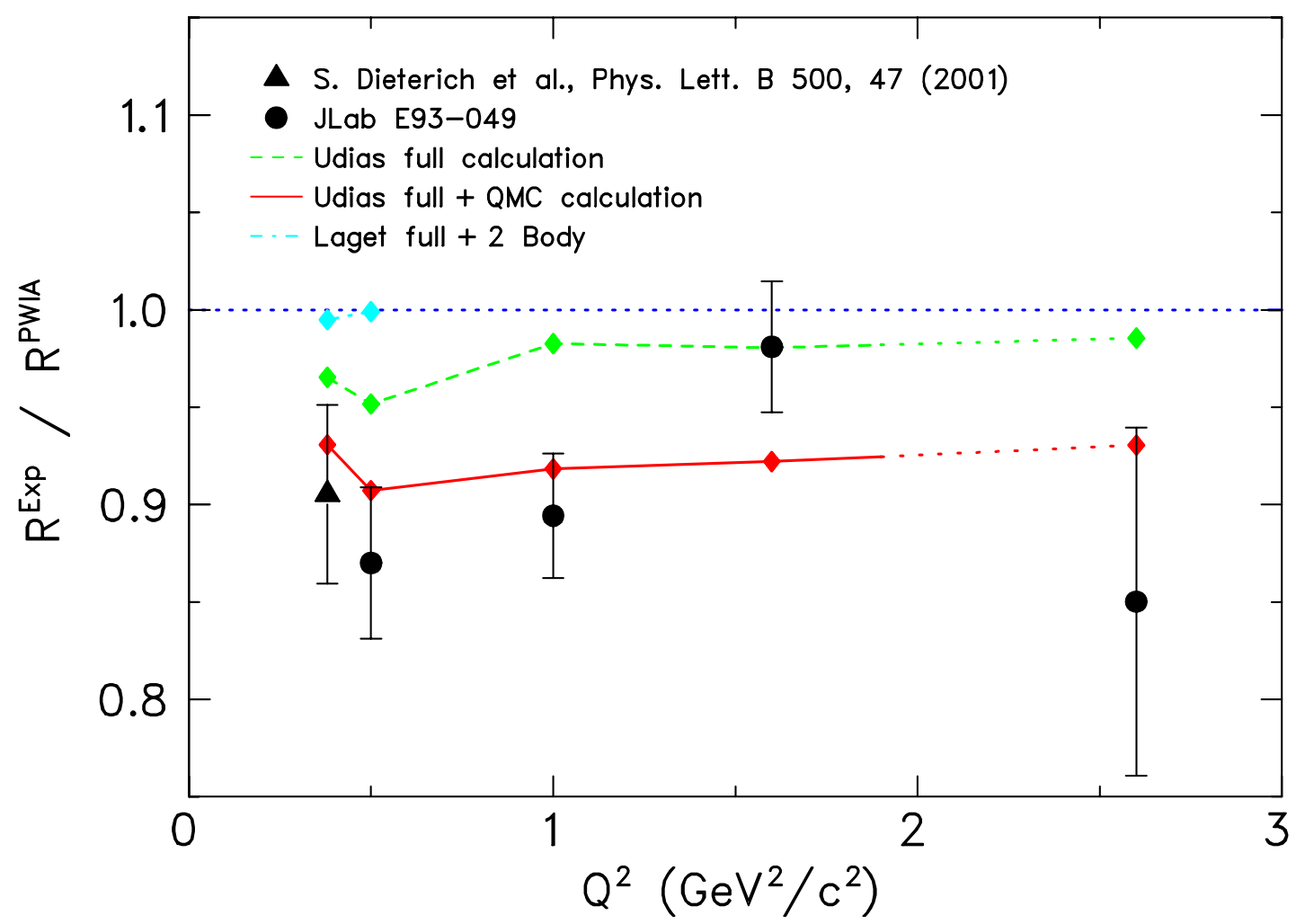

Figure 6.6: Super-Ratio $R^{\exp } / R^{\text {PWIA }}$ for all $Q^{2}$ compared to phase space averaged theoretical predictions (diamond symbols). Diamonds connected by the dash-dotted line represent Laget's calculation with two body currents. Diamonds connected by the dashed line represent the result from the full calculation by Udias; diamonds connected by the solid line represent the same calculation including medium modified form factors as calculated in the QMC model. 


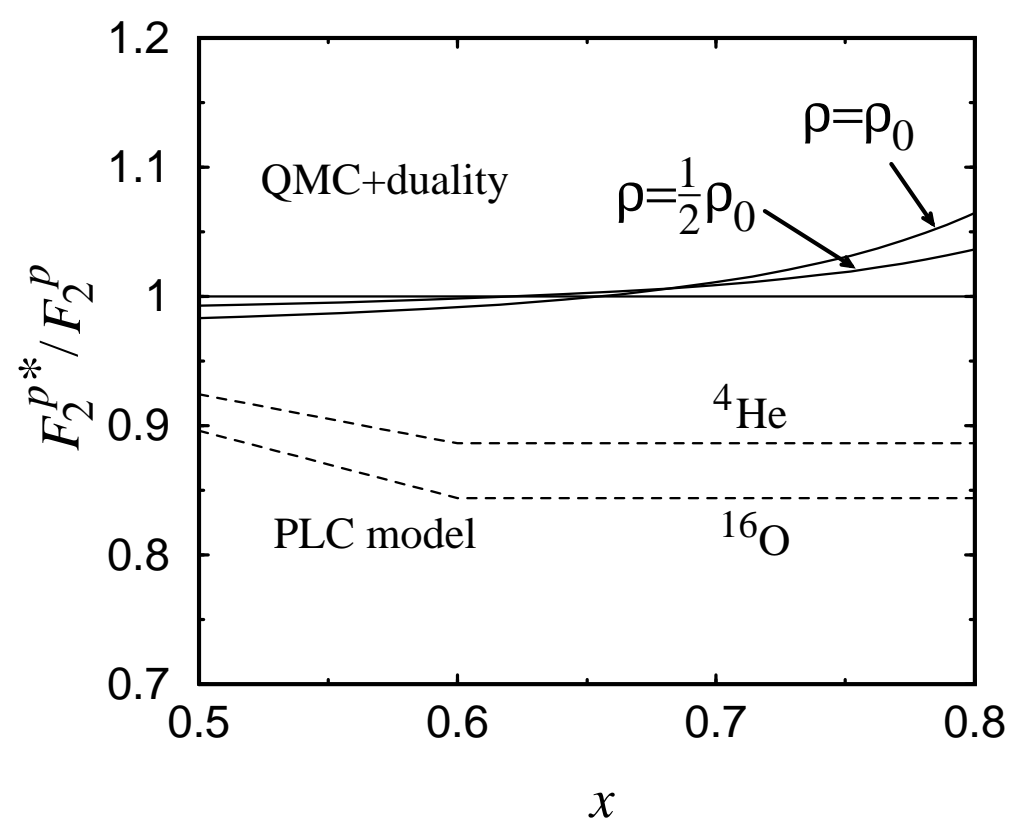

Figure 6.7: In-medium to free proton F2 structure function as function of $x$ at threshold, $x=$ $x_{\mathrm{th}}$, extracted from the Mainz data within the QMC model and local duality, at nuclear matter density $\rho=\rho_{0}$, and at $\rho=\frac{1}{2} \rho_{0}$ (solid lines). For comparisons the results of the PLC suppression model [88] are shown for ${ }^{4} \mathrm{He}$ and ${ }^{16} \mathrm{O}$ (dashed lines). Figure taken from [86].

structure function measured in inclusive lepton scattering at low mass of the hadronic final state $W$ follows a global scaling curve which describes high $W$ data. Furthermore, duality appears to hold locally in that the averaged resonance and scaling structure functions are equal for each resonance region over restricted intervals of $W$. Using this local scaling one can also test the self-consistency of the theoretical models. The form factor datum from Mainz places strong constraints on the medium modification of inclusive structure functions at high $x$. In particular, models in which the bulk of the nuclear EMC effect is attributed to the deformation of the intrinsic off-shell nucleon structure are strongly disfavored. Figure 6.7 shows the in-medium to free proton F2 structure function as function of $x$ at threshold, $x=x_{\text {th }}$, extracted from the Mainz data within the QMC model and local duality, for two nuclear matter densities. For comparison the results of the PLC suppression model [88] are shown for ${ }^{4} \mathrm{He}$ and ${ }^{16} \mathrm{O}$. 


\section{Chapter 7}

\section{Conclusion and Outlook}

The polarization transfer ratio $P_{x}^{\prime} / P_{z}^{\prime}$ was measured in ${ }^{1} \mathrm{H}\left(\vec{e}, e^{\prime} \vec{p}\right)$ at $Q^{2}$ of $0.4,0.5$, 1.0 and $1.6 \mathrm{GeV}^{2}$ and in ${ }^{4} \mathrm{He}\left(\vec{e}, e^{\prime} \vec{p}\right)^{3} \mathrm{H}$ at $Q^{2}$ of $0.4,0.5,1.0,1.6$ and $2.6 \mathrm{GeV}^{2}$. The measured ratio of the electric to magnetic form factor in ${ }^{1} \mathrm{H}\left(\vec{e}, e^{\prime} \vec{p}\right)$ calibration data is in good agreement with previous polarization transfer data. At lower $Q^{2}$, the hydrogen data also agrees well with unpolarized measurements. The results of the helium data were compared to several theoretical models. The most basic model was a PWIA calculation. The model by Laget was used to study the influence of IC and MEC interactions. The last set of models consisted of the PWIA, RDWIA and RDWIA+QMC calculations by Udias.

Polarization transfer data in ${ }^{4} \mathrm{He}\left(\vec{e}, e^{\prime} \vec{p}\right)^{3} \mathrm{H}$ disagree with PWIA calculations and also show a significant deviation from DWIA. The deviations can possibly be explained by proton medium modifications. Influences from MEC and IC as calculated by Laget were shown to be small for $Q^{2}=0.4 \mathrm{GeV}^{2}$. They are expected to become even smaller at higher $Q^{2}$, where calculations are not yet available. The theoretical models of Udias show only a very small sensitivity to the chosen bound state wave function. Of the two most commonly used relativistic current operators, $c c 1$ and $c c 2, c c 1$ gives a significantly lower value of $R$. Using $c c 1$ improves the fit to the data, but the predicted values for $R$ are still systematically higher than the data. Within the models of Udias and the assumption that the effects of IC and MEC are also small at higher $Q^{2}$, the inclusion of a medium modified form factor gives the best agreement with the data.

The theoretical interpretation is ongoing with more calculations underway. Future projects are to include MEC into the Madrid code and obtaining calculations for modified form factors other than the QMC model. The influence of optical models on R will 
be studied. In the previous chapter, constraints of the measured form factor ratio on models trying to explain the EMC effect by using local duality have been discussed. The calculations by Laget will be extended to cover higher $Q^{2}$. Ryckebusch and Debruyne are currently using the Eikonal approximation to get a better theoretical understanding of the data.

For the future, several approaches could be used to put experimental constraints on various parameters of the theoretical models. The high precision and statistics of the ${ }^{1} \mathrm{H}\left(\vec{e}, e^{\prime} \vec{p}\right)$ calibration data of this experiment allows to determine the false asymmetries in the polarimeter to a level of $1-2 \%$. Extraction of single polarizations could be used to measure $P_{n}^{\prime}$, which constrains optical models. The study of polarization transfer in heavier nuclei, e.g. ${ }^{16} \mathrm{O}$, could provide more insight into possible medium effects, because separating the proton knockout from different shells allows to study the explicit density dependence of the effect, and the dependence on different spin-orbit configurations. The experiment should be done at momentum transfers where cross section data are already available, so cross section and polarization constraints can be applied simultaneously.

In summary, this experiment has shown evidence for departure of $R$ from plain wave and nonrelativistic calculations. Even the fully relativistic calculation by Udias is not in full agreement with the data. It is possible to explain the deviation using medium modifications as predicted by the QMC model. Further theoretical and experimental investigation, extending to other nuclei and coordinating cross section and polarization transfer measurements, will enhance our understanding of this long discussed problem in nuclear physics. 


\section{References}

[1] A. I. Akhiezer, M. P. Rekalo, Sov. J. Particles Nucl. 3, 277 (1974).

[2] R. G. Arnold, C. E. Carlson, F. Gross, Phys. Rev. C 23, 363 (1981).

[3] B. Milbrath et al., Phys. Rev. Lett. 82, 2221 (1999); M.K. Jones et al., Phys. Rev. Lett. 84, 1398 (2000); O. Gayou et al., Phys. Rev. C, 038202 (2002).

[4] J.E. Ducret et al., Nucl. Phys. A556, 373 (1993); K.I. Blomqvist et al., Z. Phys. A351, 353 (1995).

[5] I. Sick, Comments Nucl. Part. Phys. 18, 109 (1988).

[6] D.B. Day et al., Ann. Rev. Nucl. Sci 40, 357 (1990); D.B. Day et al., Phys. Rev. Lett. 59, 427 (1987).

[7] J. J. Kelly, Nucleon Knockout by Intermediate Energy Electrons, Adv. Nucl. Phys. 23, 75 (1996).

[8] F. Halzen, A. D. Martin, Quarks and Leptons, John Wiley \& Sons, New York (1984).

[9] R. G. Sachs, Phys. Rev. 119, 1105 (1960).

[10] M. N. Rosenbluth, Phys. Rev. 79, 615 (1950).

[11] M.K. Jones et al., Phys. Rev. Lett. 84, 1398 (2000).

[12] S. Boffi, C. Giusti, F.D. Pacati, and M. Radici, Electromagnetic Response of Atomic Nuclei, p. 426, Oxford University Press, Oxford (1996).

[13] A. Picklesimer, J. W. Van Orden, Phys. Rev. C 35, 266 (1987).

[14] T. W. Donnelly, in Proceedings of the Workshop on Perspectives in Nuclear Physics at Intermediate Energies, edited by S. Boffi, C. C. degli Atti, and M. Giannini, Trieste, 1984, World-Scientific.

[15] A. S. Raskin and T. W. Donnelly, Ann. Phys. (N.Y.) 191, 78 (1989).

[16] J. Kelly, LEA User's Manual, internal report, 1996; available at the LEA home page at http://www.nscp.umd.edu/ kelly/LEA/lea.html.

[17] J.M. Udias, J.A. Caballero, E. Moya de Guerra, J.E. Amaro and T.W. Donnelly, Phys. Rev. Lett. 83, 5451 (1999); J.M. Udias and J.R. Vignote, Nucl. Phys. A632, 323 (1998).

[18] J.M. Laget, Nucl. Phys. A579, 333 (1994). 
[19] S. Strauch, private communication.

[20] T. de Forest, Nucl. Phys. A392, 232 (1983).

[21] B. D. Serot and J. D. Walecka, Adv. Nucl. Phys. 16, 1 (1986).

[22] J. M. Udias and J. R. Vignote, Phys. Rev. C 62, 034302 (2000).

[23] J. Ryckebusch, D. Debruyne, W. Van Nespen and S. Janssen, Phys. Rev. C 60, 034604 (1999).

[24] J.-M. Laget, Nucl. Phys. A 579, 333 (1994).

[25] J. Aubert et al., Phys. Lett. B 123, 123 (1983).

[26] I. Sick, D. Day and J.S. McCarthy, Phys. Rev. Lett. 45, 871 (1980).

[27] J. Jourdan, Phys. Lett. B 353, 189 (1995).

[28] P. J. Mulders, Phys. Rev. Lett. 54, 2560 (1985).

[29] R. Barreau et al., Nucl. Phys. A402, 515 (1983).

[30] R. Friedberg and T. D. Lee, Phys. Rev. D 15, 1694 (1977).

[31] L. S. Celenza, A. Rosenthal and M. C. Shakin, Phys. Rev. C 31, 212 (1985);

L. S. Celenza, A. Rosenthal and M. C. Shakin, Phys. Rev. C 31, 232 (1985).

[32] M. R. Frank, B. K. Jennings, G. A. Miller, Phys. Rev. C 54, 920 (1996).

[33] F. Schlumpf, "Relativistic constituent Quark Model For Baryons", Doctoral thesis, 1992, Report No. hep-ph/9211255; F. Schlumpf, Phys. Rev. D 50, 6895 (1994); F. Schlumpf, Phys. Rev. D 51, 2262 (1995); S. J. Brodsky, F. Schlumpf, Phys. Lett. B 329, 111 (1994).

[34] D.H. Lu et al., Phys. Lett. B 417, 217 (1998); A.W. Thomas et al., nuclth/9807027; D.H. Lu et al., Phys. Rev. C 60, 068201 (1999).

[35] A. W. Thomas, W. Weise, The Structure of the Nucleon, Wiley-VCH, Berlin, (2001).

[36] H. Herminghaus et al., Nucl. Instr. Meth. 138,1 (1976); H. Herminghaus and H. Euteneuer, Nucl. Instr. Meth. 163,299 (1979); H. Herminghaus et al., Proc. LINAC Conf. 1990, Albuquerque, New Mexico, Los Alamos National Laboratory Report LA-12004-C (1990).

[37] H. Euteneuer et al., Beam measurements and operating experience at MAMI, in Proceedings of the 12th International Symposium on High-energy Spin Physics (SPIN96), Amsterdam, (1996).

[38] P. Hartmann, Doctoral Thesis, Mainz University, Germany (1998).

[39] K. Aulenbacher et al., High Capture Efficiency for the Polarized Beam at MAMI by R.F.-Synchronized Photoemission, Proc. of the 1998 European Particle Accelerator Conference, Stockholm, 22.-26.6.98, p. 1388. 
[40] J. Bermuth, Diploma Thesis, Mainz University, Germany (1996).

[41] H. Euteneuer et al., Beam Measurements and Operating Experience at MAMI, Proc. of the 1994 European Particle Accelerator Conference, London, 27.6. 1.7.94, Vol.1, p. 506.

[42] T. Doerk et al., Methods for High Precision Beam Energy Measurement at the Mainz Microtron, Proc. of the 1996 European Particle Accelerator Conference, Barcelona, 10.6. - 14.6.96, p. 1405.

[43] K. Aulenbacher et al., New Installations and Beam Measurements at MAMI, Proc. of the 1998 European Particle Accelerator Conference, Stockholm, 22.-26.6.98, p.523.

[44] K.I. Blomqvist et al., Nucl. Instr. Meth. A403, 263 (1998).

[45] G. Rosner, Habilitation Thesis, Mainz University, Germany (1995).

[46] M. Kohl, Doctoral Thesis, Darmstadt University of Technology, Germany (2001).

[47] I. Ewald, Doctoral Thesis, Mainz University, Germany (2000).

[48] T. Pospischil, Doctoral Thesis, Mainz University, Germany (2000).

[49] A. Amroun et al., Nucl. Phys. A579, 596 (1994).

[50] M. Kuss, Doctoral Thesis, Darmstadt University of Technology, Germany (1996).

[51] D.J. Margaziotis and K. Aniol, private communication.

[52] T. Pospischil et al., submitted to NIM.

[53] http://wwwa1.kph.uni-mainz.de/A1/magnet.html.

[54] A. Richter, Doctoral Thesis, Mainz University, Germany (1994).

[55] K. W. Krygier, Doctoral Thesis, Mainz University, Germany (1996).

[56] V. Kunde, Doctoral Thesis, Mainz University, Germany (1996).

[57] H. Kramer, Doctoral Thesis, Mainz University, Germany (1995).

[58] C. Sinclair, in: Proceedings of the 1999 Particle Accelerator Conference, New York,IEEE, New York, 1999, p.65.

[59] J. Berthot and P. Vernin, Nucl. Phys. News 9, 12 (1990); O. Ravel, Doctoral Thesis, University of Blaise Pascal, Clermont-Ferrand (1997).

[60] http://www.jlab.org/ moller/index.html.

[61] http://www.jlab.org/compton/.

[62] http://hallaweb.jlab.org/equipment/high_resol.html.

[63] K. Fissum, VDC Manual, MIT-LNS $\sharp 03$, Internal Report (1997). 
[64] http://hallaweb.jlab.org/equipment/trigger_electronics.html.

[65] K. Wijesooriya, Ph.D. thesis, College of William and Mary (1999), unpublished.

[66] CODA User's Manual, Internal Report, Jefferson Lab, (1995).

[67] E. Offerman, ESPACE User's Guide, Internal Report, Jefferson Lab, (1996).

[68] S. Malov, Ph.D. thesis, Rutgers University (1999), unpublished.

[69] W.R. Leo, Techniques for Nuclear and Particle Physics Experiments,p. 38, Springer, Berlin, (1994).

[70] B. Reitz, private communication.

[71] N. Liyanage, private communication.

[72] L. Bimbot et al., currently under preparation.

[73] K. Herter, Diploma Thesis, Mainz KPH 04/92, unpublished.

[74] E. Aprile-Giboni et al., Nucl. Instr. Meth. 215, 147 (1983).

[75] B. Bonin et al., Nucl. Instr. Meth. A288, 379 (1990).

[76] R.D. Ransome et al., Nucl. Instr. Meth. 201, 315 (1982).

[77] P. Vernin, G. Quemener, private communication.

[78] M. Berz, Cosy Infinity User's Manual, MSU, unpublished, 1996; http://www.beamtheory.nscl.msu.edu/cosy.

[79] D. Besset et al., Nucl. Instr. Meth. 166, 515 (1979).

[80] D.H. Lu, A.W. Thomas, A.G. Williams, Phys. Rev. C 57, 2628 (1998).

[81] P. Mergell, U.G. Meissner, and D. Drechsel, Nucl. Phys. A596, 367 (1996).

[82] P. Kroll, M. Schürmann and W. Schweiger, Z. Phys. A 338, 339 (1991).

[83] P.L. Chung and F. Coester, Phys. Rev. D 44, 229 (1991); I.G. Aznauryan, Phys. Lett. B 316, 391 (1993).

[84] http://www.physics.odu.edu/ ulmer/mceep/mceep.html.

[85] J.M. Udias, private communication.

[86] W. Melnitchouk, K. Tsushima and A.W. Thomas, nucl-th/0110071.

[87] E.D. Bloom and F.J. Gilman, Phys. Rev. Lett. 16, 1140 (1970); Phys. Rev. D 4, 2901 (1971).

[88] L.L. Frankfurt and M.I. Strikman, Nucl. Phys. B250, 1595 (1985); L.L. Frankfurt and M.I. Strikman, Phys. Rep. 160, 235 (1988); M. Sargsian, L.L. Frankfurt and M.I. Strikman, Z. Phys. A 335, 431 (1990). 


\section{Acknowledgments}

I want to thank my adviser Ron Ransome for his support during my time as his graduate student. He always made time for many helpful discussions and provided his guidance throughout my work at this project. In addition, he gave me numerous opportunities to present my research at national and international conferences and workshops.

I would like to thank my thesis committee members, Ron Ransome, Ron Gilman, John Conway, Wim Kloet and Paul Ulmer for stimulating and thoughtful discussions.

Many thanks also to the director of the Rutgers graduate program, Prof. Jolie Cizewski, who provided guidance and advice during my time as graduate student. I thank also the graduate secretaries Kathy DiMeo and Phyllis Ginsberg, and Patty Gulyas, who were of tremendous help dealing with all administrative issues.

It was a pleasure to work with the Rutgers intermediate energy nuclear physics group: Charles Glashausser, Ron Ransome, Ron Gilman, Gerfried Kumbartzki, Steffen Strauch, Xiadong Jiang, Sergey Malov and Fatiha Benmokhtar. In addition to many discussions about physics, they provided an enjoyable work environment. They always provided help when help was needed.

My thanks to Prof. Thomas Walcher and the spokespersons of the Mainz experiment, Prof. Gunther Rosner, Prof. Hartmut Schmieden, Prof. Reiner Neuhausen and Prof. Ron Ransome. They provided me the opportunity to take part in an exciting experiment. Special thanks also to Dr. Thomas Pospischil and his dedication to the Mainz FPP project. I would also like to thank the A1 graduate students, staff and technicians were giving us great support during preparation, data taking and analysis of the Mainz experiment. Special thanks to all of you who took shifts, spent long evenings preparing the target, and answered numerous questions about software and analysis. 
I would like to thank the spokespersons of E93-049, Prof. Rolf Ent and Prof. Paul Ulmer, for the opportunity to work with them. My thanks also to the members of the FPP, E93-049 and JLab Hall A collaborations for their help during the experiment and analysis. Many thanks to the technicians of Hall A for their support during the experiment.

I would like to thank all my friends who made Newport News a home for me. First and foremost my thanks to Bill Blair and the Newport News congregation. Their prayers, support and friendly welcome (including marvellous Thanksgiving dinners at the Sassenhagen residence!) helped me through many stressful times. Corinne, Laeticia and Peter Hartmann always opened their joyful home to me. Krishni Wijesooriya, Nilanga Lianage, Sergey Malov, Fatiha Benmokhtar, Laura Marcucci, Michael Kuss and Bodo Reitz were good friends and companions.

I am very grateful for my former colleague and partner Steffen Strauch. Without his unwaving support in most difficult times this work could not have been accomplished. Nimmer vergeht, was Du liebend getan.

Meiner Familie und meinen Freunden in Deutschland möchte ich für all die guten Wünsche, Ratschläge und ihre Unterstützung während dieser Arbeit danken. Sie waren immer für mich da, wenn sie gebraucht wurden. Ganz besonderen Dank an meine liebe Freundin Irmi mit dem grossen Herzen, die mir in ihrer Kraft und Entschlossenheit, ihrem Mut wider alle Wiederstände ihren eigenen Weg zu gehen und ihrer Lust and der Forschung immer ein grosses Vorbild war. 


\section{VITA}

\section{Sonja Dieterich}

1993-1998 Major in Physics, Technical University of Darmstadt, Darmstadt, Germany.

1998 M.Sc. in Physics, Technical University of Darmstadt, Darmstadt, Germany Thesis Title: "Kalibrierung des QCLAM Spektrometers am S-DALINAC".

1998-2002 Graduate work in Physics, Rutgers, The State University of New Jersey, New Brunswick, New Jersey, U.S.A.

2002 Ph.D. in Physics, Rutgers, The State University of New Jersey, New Brunswick, New Jersey, U.S.A.

Thesis Title: "Polarization Transfer in the Reaction ${ }^{4} \mathrm{He}\left(\vec{e}, e^{\prime} \vec{p}\right)^{3} \mathrm{H}$ in the Quasielastic Scattering Region".

2002-present Postdoctoral Fellow, Georgetown University, Washington D.C., U.S.A.

2001 "Polarization Transfer in the ${ }^{4} \mathrm{He}\left(\vec{e}, e^{\prime} \vec{p}\right)^{3} \mathrm{H}$ Reaction", S. Dieterich et al., (nucl-ex/0011008), Phys. Lett. B 500, 47 (2001).

2001 "Polarization Transfer in the ${ }^{4} \mathrm{He}\left(\vec{e}, e^{\prime} \vec{p}\right)^{3} \mathrm{H}$ Reaction", S. Dieterich, Proc. Int. Symposium on Nuclei and Nucleons, Darmstadt, Germany, 11 October 2000, Nucl. Phys. A 690, 231 (2001).

2001 "Polarization Measurements in High-energy Deuteron Photodisintegration", K. Wijesooryia et al., Phys. Rev. Lett. 86, 2975 (2001).

2002 "Measurement of $G_{E_{p}} / G_{M_{P}}$ in $\vec{e} p \rightarrow e \vec{p}$ to $Q^{2}=5.6 \mathrm{GeV}^{2}$ ", O. Gayou et al., Phys. Rev. Lett. 88, 092301-1 (2002). 